\title{
Inter-subunit Crosstalk via PDZ Synergistically Governs Allosteric Activation of Proapoptotic HtrA2
}

Aasna L. Parui, ${ }^{1,2}$ Vandana Mishra, ${ }^{3}$ Shubhankar Dutta, ${ }^{1}$ Prasenjit Bhaumik, ${ }^{3}$ Kakoli Bose ${ }^{1,2, *}$

${ }^{1}$ Advanced Centre for Treatment, Research and Education in Cancer (ACTREC), Tata Memorial Centre, Kharghar, Navi Mumbai - 410210, India.

${ }^{2}$ Homi Bhabha National Institute, BARC Training School Complex, Anushaktinagar, Mumbai - 400094, India.

${ }^{3}$ Department of Biosciences and Bioengineering, Indian Institute of Technology Bombay, Powai, Mumbai - 400076, India.

*Corresponding author

Correspondence: kbose@actrec.gov.in 


\section{SUMMARY:}

Mitochondrial serine protease - High temperature requirement A2 (HtrA2), is associated with various diseases including neurodegenerative disorders and cancer. Despite availability of structural details, the reports on HtrA2's mechanistic regulation that varies with the type of activation signals still remain non-concordant. To expound the role of regulatory PDZ domains in promoting synergistic coordination between HtrA2 subunits, we generated heterotrimeric HtrA2 variants comprising different numbers of PDZs and/or active-site mutations. Sequential deletion of PDZs from the trimeric ensemble significantly affected its residual activity in a way that proffered a hypothesis advocating intermolecular allosteric crosstalk via PDZ domains in trimeric HtrA2. Furthermore, structural and computational snapshots affirmed the role of PDZs in secondary structural element formation and coordinated reorganization of the N-terminal region and regulatory loops. Therefore, apart from providing cues for devising structure-guided therapeutic strategies, this study establishes a physiologically relevant working model of complex allosteric regulation through a multifaceted trans-mediated cooperatively-shared energy landscape.

KEYWORDS: allostery, HtrA2, ensemble, intermolecular crosstalk, PDZ, trans-mediated activation 


\section{INTRODUCTION:}

Cells respond to different environmental cues by channelizing distinct signal transduction pathways, often controlled by conformational adaptations of multidomain allosteric enzymes. The modulations include subtle loop movements and/or larger domain reorientations upon ligand binding at the allosteric pocket, leading to enhanced substrate catalysis [1, 2]. Interestingly, these conserved regulatory domains that confer a distinct spatial scaffold to the overall structure have been evolutionarily tuned to perform specific functions in several unrelated proteins by accommodating themselves to global structural plasticity in diverse macromolecular milieu [3, $4]$.

PDZ (Postsynaptic density-95/Discs large/Zonula occludens-1) domains are modular proteinprotein interaction moieties specialized for binding to C-terminal motifs of various proteins [57]. The multiple ligand-binding sites in PDZ contribute toward their unparallel conformational plasticity relay signals from a distal binding region to the functional site. Human HtrA2 (High temperature requirement protease A2), a PDZ-bearing mitochondrial proapoptotic serine protease that is associated with neurodegeneration and cancer, is an important therapeutic target $[8,9]$. Despite exhibiting evolutionarily conserved higher-order architecture with one or two PDZs and a well-defined active site pocket [10], prevalence of low sequence identity and ligandinduced distinct structural signatures provide the basis for the specificity and functional diversity in the HtrA family [9, 11]. One of the unique features of HtrA2 is removal of N-terminal transmembrane region upon apoptotic induction. This leads to its cytoplasmic translocation and subsequent cleavage of inhibitor of apoptosis proteins (IAPs) through an exposed AVPS motif thus promoting cell death through a binary activation mode [12]. Furthermore, HtrA2 promotes non-classical caspase-independent apoptosis [13, 14] via its intricate serine protease activity. 
However, the complex mechanism through which it promotes initiation of apoptotic cascades or regulates protein quality control remains elusive.

The crystal structure of the catalytically inactive apo-HtrA2 [15] proposed a mechanistic model wherein the otherwise catalytically competent protease domain are stereochemically shielded by PDZ from the same monomer in its basal state thus implicating a cis-mediated inhibitory role of PDZs. However, subsequent reports that demonstrated a significant decrease in the protease activity of a PDZ-lacking variant $[16,17]$ as well as multiple activation routes of HtrA2 [18-21] challenged this pre-existing tenet. Revisitation of the mode of HtrA2 activation highlighted a dual regulatory inter-molecular switch (through allosteric ligand-binding to PDZs or N-terminus) promoted coordinated structural reorganizations at the regulatory loops essential for HtrA2 activation [17, 22]. However, these studies as well as multiple structural reports [15, 18, 23] fail to elucidate the intricate real-time conformational dynamics with respect to different modes of activation viz. substrate binding or temperature thus leaving a huge vacuity toward understanding the multifaceted role of HtrA2.

Evolutionarily, PDZs are known to govern the active and/or inactive state(s) of HtrA oligomers. Apart from the N-terminal-mediated regulation in HtrA2 [22], the transition from apo-form to the proteolytically active state seems to be strictly governed by PDZs, owing to its specific interactions with numerous C-terminal binding partners such as cytoskeletal proteins and other apoptosis-related molecules. The basic requirement of HtrA proteases to exist as large oligomeric ensembles, despite having all the functional elements present in a single subunit, is quite astonishingly profound. The aim of this study is to recognize the contribution of each HtrA2 subunit towards the dynamic energy landscape within the trimeric ensemble and to identify how individual PDZs, besides allosterically regulating HtrA2 function, help attain an 
active functional state upon multimodal stimulation. To achieve this, we generated different heterotrimeric HtrA2 variants that lack one or two PDZ domains and performed comprehensive enzyme kinetic analyses of each variant with a C-terminal binding partner. Through active-site chemical profiling [24], we established that binding of substrate to a single PDZ not only significantly affects the proteolytic activity of adjacent subunits, but also compensates for the loss of active-sites within the ensemble. In case of both substrate- and temperature-based activations, we demonstrated an intricate predominant interdomain networking (trans PDZprotease crosstalk) that leads to subtle reorganization of the active-site loops and formation of a protease-substrate complex that is tuned for efficient catalysis. This networking is responsible for maintaining the overall proteolytic activities of each HtrA2 subunit via cooperatively-coupled energy dynamism within the trimeric ensemble. Furthermore, this study emphasizes the importance of the evolutionarily conserved PDZ in determining the active functional state of HtrA2 that is catalytically more competent under a specific cellular scenario and is also energetically most favorable. The concept encompassing such functionally important events, which can be transmitted long-range to affect the affinity or catalytic efficiency of a distant active-site, poses a challenge and necessity to identify the communication pathway underlying the functional effects of such larger macromolecular assemblies within the cell.

\section{RESULTS:}

\section{Generation of heterotrimeric HtrA2 variants with different number of PDZ domains}

For understanding the specific function of PDZs in propagating allosteric communication, we generated heterotrimeric variants of HtrA2, which differed in the number of PDZs. The constructs used include the homotrimeric His6-tagged wildtype (WT or WWW) and the His3- 
tagged $\Delta \mathrm{PDZ}$ construct (N-SPD or $\Delta \Delta \Delta$ ) (Fig. 1A). The purified homotrimers were mixed in equimolar concentrations and subjected to mild denaturing conditions. Subsequent renaturation led to the formation of four plausible HtrA2 variant combinations - two homotrimers (WWW, $\Delta \Delta \Delta$ ) and two heterotrimers (WW $\Delta, \mathrm{W} \Delta \Delta$ ) (Fig. 1A). The additional N-terminal FLAG-tag in $\Delta \Delta \Delta$ imparted $\sim 0.2$ difference in $\mathrm{pI}$ (as estimated by ProtParam software) [25], thus enabling the variants in the renatured mixture to be well resolved in native PAGE (Fig. 1B). Furthermore, when probed with anti-FLAG antibody, three out of four bands (having $\Delta$ subunit) were spotted with varying intensities (different number of FLAG epitopes), confirming the distinctiveness of the heterotrimers (Fig. 1C). These renatured variants were further separated using a modified version of IMAC (Immobilized metal affinity chromatography), based on the differential affinity toward histidine tag. As $\mathrm{W}$ and $\Delta$ subunit differed in the number of C-terminal histidine tag residues, batch purification with a narrow imidazole gradient led to the elution of $\Delta \Delta \Delta$ in flowthrough, followed by $\mathrm{W} \Delta \Delta$, WW $\Delta$, and WWW eluting between 50-250 mM imidazole gradient (Fig. 1D). The heterotrimers obtained were stable, as estimated by native PAGE (Fig. 1E). The purity of WW $\Delta$ and $\mathrm{W} \Delta \Delta$ were found to be slightly lower ( 90\%) as compared to the other variants due to interference from the adjoining variant. For further enzymatic studies, the homotrimers were purified, denatured and renatured separately.

\section{Presence of single or multiple PDZ domains influences HtrA2 proteolytic activity}

To understand the effect of PDZ deletion on the overall residual activity and specificity of HtrA2 variants, time-based proteolytic assays were performed using the generic substrate $\beta$-casein. This substrate has a putative binding site (GPFPIIV) that interacts with 'YIGV' groove of HtrA2 PDZ [16] and mimics the canonical PDZ-mediated allosteric modulation. It was observed that although the HtrA2 variants exhibited similar cleavage pattern (with three distinct bands), the 
cleavage time differed significantly with removal of each PDZ from the trimer (Fig. 2A). While variants having at least one PDZ (WWW, WW $\Delta$ and $\mathrm{W} \Delta \Delta$ ) cleaved within 10 min of incubation with a serial decline in the cleavage rate with PDZ deletion, $\Delta \Delta \Delta$ displayed significant reduction of activity with 100\% substrate cleavage only by 60 min (Fig. 2B). For quantitative evaluation, fluorometric analysis was performed using varying concentrations of FITC-labeled $\beta$-casein as a substrate. Interestingly, a decreasing trend for $\mathrm{V}_{\max }$ was also observed with removal of each PDZ domain. WWW displayed the highest $\mathrm{V}_{\max }$, followed by WW $\Delta$ and $\mathrm{W} \Delta \Delta$, which demonstrated approximately two- and four-fold decrease respectively, while $\Delta \Delta \Delta$ showed a $\sim 10$-fold decrease in $\mathrm{V}_{\max }$ (Fig. 2C and Table 1).

Although PDZ deletion resulted in only minor changes in the positive co-operativity of heterotrimers, the apparent sequential decline in $\mathrm{V}_{\max }$ proposes a strong possibility of intersubunit crosstalk between PDZ and adjacent protease domain, which eventually influences the second step of enzyme kinetics i.e. substrate catalysis. Alternatively, $\Delta \Delta \Delta$ exhibited maximum substrate binding affinity $\left(\mathrm{K}_{\mathrm{m}}\right)$, followed by $\mathrm{W} \Delta \Delta$ and $\mathrm{WW} \Delta$. This is expected as removal of PDZ inhibition is known to open up the base of the pyramidal ensemble [15, 26], thereby increasing substrate accessibility leading to pseudo-affinity exhibited by enzyme. Despite this, we observed a marked decrease in $\mathrm{V}_{\text {max }}$ and substrate turnover $\left(\mathrm{k}_{\mathrm{cat}}\right)$ rates for $\mathrm{WW} \Delta, \mathrm{W} \Delta \Delta$ and $\Delta \Delta \Delta$. These results hint toward the presence of a malformed oxyanion hole in PDZ-lacking variants that also corroborates well with our previous studies [16, 17]. To assess the relative orientation of oxyanion hole residues, comparative 100 ns MDS analyses of these $\Delta$-containing variants were performed, keeping WWW as the reference structure. In-depth structural comparison revealed a $90^{\circ}$ anti-clockwise flip in the imidazole ring of F303 away from the catalytic triad residue $\mathrm{H} 198$ for WW $\Delta, \mathrm{W} \Delta \Delta$ and $\Delta \Delta \Delta$ (Fig. S1). This distal movement disrupts 
the nucleophilic exchange between F303 and H198 that is indispensable for stabilizing the negatively charged intermediates generated due to H198-induced deprotonation of the catalytic serine S306 [9, 16]. This further explains the decrease in catalytic efficiencies, with $\Delta \Delta \Delta$ showing approximately four-fold, while $\mathrm{W} \Delta \Delta$ and WW $\Delta$ approximately two- and $\sim 1.5$-fold decrease respectively, as compared to WWW. Overall, these results suggest that all the three PDZs of the HtrA2 trimeric ensemble govern its proteolytic activation by maintaining proper orientation of the catalytic triad, which is a prerequisite for a stabilized transition state formation and subsequent substrate catalysis.

\section{Catalytic pocket reorientation and structural perturbation captured through MDS}

To investigate the role of PDZ in modulating substrate accessibility, in silico docking analysis was performed using $\beta$-casein peptide (GPFPIIV) and HtrA2 variants. Comparison of the apparent binding affinities showed $\Delta \Delta \Delta$ having higher docking score than WW $\Delta$ and W $\Delta \Delta$ (Table S1). Interaction analysis of the docked complexes revealed the propensity of the peptide to bind with H394, P409 and G410 of PDZ (Figs. S2A, S2B and S2C). However, due to higher accessibility in $\Delta \Delta \Delta$, the peptide directly forms interactions with the active-site residues (H198, D228 and S306), resulting in higher binding score compared to the PDZ-containing variants (Fig. S2D). Furthermore, to discern the underlying mechanism contributing to differences in catalytic efficiency, MDS analyses were performed with these docked complexes. Previous reports showed that HtrA2 undergoes catalytic triad reorientation by moving H198 towards D228, and S306 away from H198, upon activator/substrate binding at the distant PDZ domain [9, 16]. Comparative distance analysis of 1000 ns MDS of the peptide-bound and unbound HtrA2 variants showed similar catalytic triad movement for WWW, resulting in successful opening of the pocket. However, with removal of PDZ, the opening of the catalytic pocket decreased 
gradually in WW $\Delta$ and $\mathrm{W} \Delta \Delta$ (Table S1 and S2). Although these movements enabled slight opening of the catalytic pocket for WW $\Delta$ and $\mathrm{W} \Delta \Delta$, the opening might not be sufficient to exhibit catalysis as efficiently as WWW and hence supports our aforementioned enzyme kinetic studies. $\Delta \Delta \Delta$, on the other hand, showed closing of the catalytic pocket upon peptide binding, as the distance between H198 and S306 was found to be decreased by $0.4 \AA, 0.5 \AA$ and $0.4 \AA$ in chains A, B and C, respectively (Table S2).

Apart from substrate-based activation, the apo-form of HtrA2 is known to be activated by increase in temperature [26], with optimum activation temperature ranging between $45{ }^{\circ} \mathrm{C}-55$ ${ }^{\circ} \mathrm{C}$ [17]. To investigate whether HtrA2 undergoes similar catalytic triad reorientation with temperature, the variants were subjected to MDS with temperature elevated to $323 \mathrm{~K}$. Temperature-induced activation also demonstrated a similar trend in opening of the catalytic pocket, with WWW showing the highest deviation of $1.4 \AA$, whereas for WW $\Delta$ and $\mathrm{W} \Delta \Delta$, the maximum deviation was not more than $0.4 \AA$ (Table S2). However, as expected, $\Delta \Delta \Delta$ demonstrated reverse movement of the active-site residues upon temperature induction that might result in improper catalytic pocket formation.

To further dissect the underlying conformational changes, metadynamics MDS was performed for the $\beta$-casein bound and temperature-afflicted forms of HtrA2 variants that generated freeenergy landscape, representing segregated conformations of the systems calculated over a collective set of catalytic triad distances [27]. In both the cases, two separate conformations were evident for WWW, corresponding to their H198-S306 or H198-D228 distances. The two lowest conformations were observed at 1.6 and $1.5 \mathrm{kcal} / \mathrm{mol}$, where the former $\Delta \mathrm{G}$ value tends to reside at higher catalytic triad residue distances when bound to the activating peptide (Fig. 3A). The two separate lowest conformations of WWW at optimum catalytic pocket distances indicate that 
opening up is a direct effect of the global conformational changes (Fig. 3B). Similar effect was observed in WWD with two discrete conformations at different free energy levels (Figs. 3C and 3D). However, for $\mathrm{W} \Delta \Delta$, though there were a few separately placed conformations, distinct energy barriers among them were missing, which is indicative of transient conformational changes that are not conducive toward enhancing its catalytic efficiency (Figs. 3E and 3F). Furthermore, in $\Delta \Delta \Delta$, single rigid conformation was observed indicating zero transitional state (Figs. 3G and 3H). This structural rigor might be due to the absence of any regulatory domain such as PDZ.

\section{Intermolecular PDZ-protease crosstalk governs different HtrA2 activation modes}

From the aforementioned studies, it is evident that extensive conformational changes with PDZ movement are imperative for the activation of HtrA2 variants. To understand the role of intraand/or intermolecular crosstalk in allosteric induction, an activity-based chemical profiling using TAMRA fluorophosphonate (TAMRA-FP) was performed. TAMRA-FP specifically labels the catalytic serine of an active serine hydrolase through covalent modification of the side chain hydroxyl group [24], thus facilitating their detection through the linked fluorescein dye. This study was envisaged to delineate how the two activation modes (substrate- or temperatureinduced) influence the active-site reactivity of the attached (cis) and/or neighboring (trans) protease domain, and the subsequent variations in the conformational dynamics upon activation.

\section{$\underline{\text { Substrate-induced activation }}$}

Upon activation, labeling of each subunit (W or $\Delta$ ) was efficiently determined through fluorescence-based SDS-PAGE. The active-site chemical profiling was performed with PDZ activator-cum-substrate $\beta$-casein. As expected, neither WWW nor $\Delta \Delta \Delta$ subunits were TAMRA- 
labeled in their basal states (Fig. S3A). Lack of modification in WWW might be due to overcrowding of PDZs in the apo-form. However, in the unbound WW $\Delta$ and $\mathrm{W} \Delta \Delta$ variants, W subunit showed $\sim 2.4$ - and $\sim 1.8$-fold increase respectively, while $\Delta$ subunit exhibited $\sim 2.8$ - and 5.3 fold respectively as compared to their respective homotrimers; thus suggesting occurrence of favorable conformational changes in and around the active-site of adjacent $\Delta$ subunit(s) with removal of PDZ(s) (Fig. S3A and S3C). On the other hand, the bound WWW and $\Delta \Delta \Delta$ complexes exhibited highest and lowest fluorescence labeling respectively (Fig. S3A). This confirms that the binding event engages PDZs and greatly alters the activity of the entire trimeric ensemble. Interestingly, $\Delta$ subunit of WW $\Delta$ showed greater modification than $\mathrm{W} \Delta \Delta$ (Fig. S3C). This might be attributed to the simultaneous influence of two PDZs on the active-site of a single $\Delta$ subunit through an intricate intermolecular PDZ-protease interplay. In $\mathrm{W} \Delta \Delta$, although the PDZ-crowding is less, the lesser fluorescence equates to modification of two $\Delta$ subunits with a single PDZ. These observations ascertain presence of a predominant trans PDZ-protease crosstalk between the ligand-bound PDZ of one subunit with the protease domain of the adjacent subunit. For quantitative validation, the TAMRA-FP intensities were compared with respect to their corresponding basal states so as to determine the fold change in active-site modification (Table S3A). While bound WWW represents 11-fold increase in TAMRA fluorescence compared to the unbound form, the W subunit of bound WW $\Delta$ and $\mathrm{W} \Delta \Delta$ exhibit five- and fourfold increase respectively (Fig. 4A). Interestingly, each $\Delta$ subunit of these heterotrimeric variants, WW $\Delta$ and $\mathrm{W} \Delta \Delta$, demonstrates increase in labeling by seven- and three-fold respectively compared to their unbound forms (Fig. 4A), thus correlating TAMRA-modification of the $\Delta$ subunit on the total number of engaged PDZs. The observed 2.5-fold fluorescence increase in the substrate-bound $\Delta \Delta \Delta$ might be attributed to greater accessibility to the active site 
in absence of PDZs that is in concurrence with the lower $\mathrm{K}_{\mathrm{m}}$ value earlier obtained for this variant.

Taken together, our studies affirm occurrence of trans-mediated allosteric communication that is extremely essential for altering the active-site environment of the trimeric ensemble.

\section{Temperature-based activation}

For understanding heat-induced PDZ-protease plasticity, similar enzyme-modification assays were carried out by incubating each variant at $50{ }^{\circ} \mathrm{C}$, a temperature at which both WWW and $\Delta \Delta \Delta$ are structurally stable with enhanced proteolytic activity [17] (Figs. S3B and S3D). Interestingly, although W subunit in WWW showed maximum modification, the difference in activity fold-change with respect to the basal state in WWW, WW $\Delta$ and $\mathrm{W} \Delta \Delta$ were quite comparable (Fig. 4B and Table S3B). Strikingly, the modification of $\Delta$ subunit was found to be inversely proportional to the number of PDZs present in each variant, with $\Delta \Delta \Delta$ exhibiting maximum change in the heat-induced activation. This apparent behavioral anomaly might be attributed to the difference in structural dynamism of the protease in the two distinct activation modes. The heat-induced activation is primarily dependent on increased plasticity of PDZs in the trimer due to increase in the overall kinetic energy of the ensemble. This might lead to enhanced movement at the regulatory loops and the PDZ-protease interface. With no substrate-mediated conformational changes, the mechanism plausibly relies predominantly on the spatial dynamism of the PDZs. Thus, while WWW and WWD experience substantial steric hindrance from the adjoining PDZs, a single PDZ of $\mathrm{W} \Delta \Delta$ remains relatively free to modify the adjacent $\Delta$ subunits. In $\Delta \Delta \Delta$, however, increase in activity might correlate with opening up of the active-site as a function of heat, which corroborates well with MDS analysis (Fig. 3H) and a previous FRET- 
based study [17]. Overall, these results hint towards a trans-mediated allosteric communication within the heat-activated HtrA2 protease.

\section{Trans PDZ-protease crosstalk predominates the allosteric communication in HtrA2}

To further validate the intermolecular communication, we generated heterotrimeric HtrA2 variants that contained inactivated protease domain in parallel with the PDZ(s). To develop such variants, we replaced active-site S306 residue(s) with alanine (denoted as 'I') in WWW and $\Delta \Delta \Delta$ constructs. Using proper subunit combinations, we generated four different heterotrimeric combinations $-\mathrm{W}_{\mathrm{I}} \mathrm{W}_{\mathrm{I}} \Delta, \mathrm{W}_{\mathrm{I}} \Delta \Delta, \mathrm{WW} \Delta_{\mathrm{I}}$ and $\mathrm{W} \Delta_{\mathrm{I}} \Delta_{\mathrm{I}}$. Comparison of the enzymatic parameters of these engineered proteases with their corresponding active counterparts (WW $\Delta$ and $\mathrm{W} \Delta \Delta$ ) showed reduction in the $\beta$-casein substrate cleavage rate. Interestingly, despite presence of two active-sites in $\mathrm{W}_{\mathrm{I}} \Delta \Delta$, it cleaved $\sim 90 \%$ of the substrate in $30 \mathrm{~min}$; while $\mathrm{W}_{\mathrm{I}} \mathrm{W}_{\mathrm{I}} \Delta$ (with a single active-site in $\Delta$ ) showed complete cleavage within the same time span (Fig. S4A). Although $\mathrm{W}_{\mathrm{I}} \mathrm{W}_{\mathrm{I}} \Delta$ exhibits similar enzymatic parameters as WW $\Delta$ (Fig. 4C), there is a marked 1.5-fold decrease in $\mathrm{V}_{\max }$ and $\mathrm{k}_{\mathrm{cat}}$ values of $\mathrm{W}_{\mathrm{I}} \Delta \Delta$ with respect to $\mathrm{W} \Delta \Delta$ (Table 1). The catalytic efficiencies of $\mathrm{W}_{\mathrm{I}} \mathrm{W}_{\mathrm{I}} \Delta$ and $\mathrm{W}_{\mathrm{I}} \Delta \Delta$ are slightly lesser than their active counterparts. Interestingly, the catalytic efficiency of $\mathrm{W}_{\mathrm{I}} \mathrm{W}_{\mathrm{I}} \Delta$ is $\sim 1.5$-fold greater than that of $\mathrm{WW} \Delta_{\mathrm{I}}$, thus strongly ascertaining the existence of trans-mediated allosteric communication via the PDZs. Moreover, $\mathrm{WW} \Delta_{\mathrm{I}}$ exhibited similar catalytic efficiency as that of $\mathrm{W} \Delta_{\mathrm{I}} \Delta_{\mathrm{I}}$, despite having two active-sites in subunits with intact PDZs. Thus, the number of active-sites within the trimeric ensemble is not the primary defining factor for the total residual activity. This is conceivably because loss of active-sites is compensated by PDZ-mediated conformational changes that might be necessary for a well-formed oxyanion hole and subsequent substrate catalysis. In both, substrate- and heatinduced activity-based chemical profiling assays, the $\Delta$ subunit in $\mathrm{W}_{\mathrm{I}} \mathrm{W}_{\mathrm{I}} \Delta$ showed slightly 
greater modification by TAMRA than $\mathrm{W}_{\mathrm{I}} \Delta \Delta$. Thus, similar observations from two distinct and independent studies strongly hint towards adoption of a unique compensatory mechanism, where the PDZ domain(s) strive(s) to indemnify the loss of activity of the attached subunit(s). Taken together, these results evidently prove the existence of a strong trans-mediated allosteric communication via a coordinated PDZ movement within the multimeric protein ensemble (Fig. 4D).

\section{Removal of PDZ affects formation of secondary structural elements}

To delineate the role of PDZ deletion on the overall structural integrity of HtrA2 trimeric ensemble, we determined the crystallographic structure of $\Delta \Delta \Delta$ (S306A) variant (PDB ID: 7VGE) at $4 \AA$ resolution (Table S4). Following the trend of relatively lower resolution structures of other available $\triangle \mathrm{PDZ} \operatorname{HtrAs}(\sim 3 \AA)$, it can be directed to the inherent dynamism and reduced compactness of the protease sans PDZ rather than any experimental deficit. Interestingly, majority of the $\triangle \mathrm{PDZ}$ structures in HtrA family displayed presence of more than one trimer molecule in the crystal asymmetric unit, probably to increase stabilization and encourage crystal packing. The structural asymmetric unit of $\Delta \Delta \Delta \operatorname{Htr} A 2$ also contains six subunits assembled into two discrete trimers (chains - $\mathrm{ABC}$ and DEF) that stack against each other in a nonsuperimposable tail-to-tail arrangement (Fig. 5A), resulting in occlusion of the funnel-shaped surface with a buried surface area of $12610 \AA^{2}$. The individual $\Delta$ subunits of each trimer are symmetrically packed around a three-fold molecular axis, with each subunit exhibiting slight variations in the catalytic and loop regions. Although overall the trimers are comparable to that of full-length HtrA2 (PDB ID: 1LCY) with main-chain backbone RMSD of $<0.45 \AA$ and both missing the sensory L3 loop, each $\Delta$ subunit displayed deviations near the activation domain and regulatory loops. Fortunately, despite relatively low resolution, the activation domain loops viz. 
LA (residues 170-174) in Chain D and L2 (residues 323-329) in Chains A and C could be successfully constructed.

\section{$\underline{\text { Inter-trimer interactions and interface analyses }}$}

Interface analysis pertaining to trimer-trimer interactions revealed that LA loop from each subunit of the upper trimer (ABC) stabilized the corresponding LA loop from the alternate subunit of the bottom trimer (DEF) (Figs. 6B and S4A). This might be the plausible reason for the two trimers to co-exist in a single asymmetric unit during crystal packing. Since PDZs are spatially close to the corresponding LA loops in the full-length structure, we speculate that absence of PDZ (especially $\beta 15-\alpha 6$ region) affects the stabilization of LA loop in $\Delta \Delta \Delta$. The association of two trimers may further assist in the stabilization of highly dynamic LA loop (Fig. 5B). In addition, the inter-trimer interactions in $\Delta \Delta \Delta$ are mostly non-bonded contacts between residues of LA, LD and L2 loop, with L2 residues (324-327) forming major non-bonded interactions in the inner core of the trimer association. Thus, in the absence of PDZs, LA, LD and L2 loops might play critical role in stabilizing the trimeric ensemble of $\Delta \Delta \Delta \mathrm{Htr} A 2$.

\section{$\underline{\text { Structural comparisons and subunit interaction analysis }}$}

Notably, LA loop in chain $\mathrm{D}$ of $\Delta \Delta \Delta$ assumed a greatly disordered conformation in contrast to the extended anti-parallel $\beta$ sheet ( $\beta$ 1 and $\beta 2$ ) observed in 1LCY (Fig. 5C). Alternatively, $\beta 7$ gained an extended $\beta$-sheet conformation with respect to the positioning of the regulatory loop LD in $\Delta \Delta \Delta$ (Figs. 6C and S4C). The $\beta 11-\beta 12$ region of L2 loop in HtrA2 (M323, V325, I329, F331) has been reported to interact with $\alpha 5$ and $\beta 14$ of PDZ (M365, L367, I373 and L377) [15]. The abrogation of this interaction in $\Delta \Delta \Delta$ resulted in reduced electron density for L2 loop residues in the $\Delta$ subunits (except chains A and C) (Figs. 6C and S4B). The deletion might have 
adversely affected the protein stability by abrogating a series of intramolecular PDZ-protease hydrophobic contacts that subsequently culminate in a disordered L2 loop with enhanced plasticity. Although the intermolecular salt bridges in most $\Delta$ subunits are similar to the wildtype structure (1LCY), PDZ deletion also affected the number of intramolecular salt bridges in $\Delta \Delta \Delta$. However, one of the conserved salt bridges between D190 (OD2) and R233 (NH2) remained intact despite PDZ deletion, thus underscoring its importance in maintaining intramolecular integrity of HtrA2 subunits. The structure depicts that all the disordered regulatory loops i.e., LA, L2 and LD are poised close to the comparatively more exposed catalytic triad in $\Delta \Delta \Delta$, than in wildtype (1LCY). Interestingly, deletion of PDZ also breaks the inter-subunit hydrogen bond between Y147-Q146 at the N-terminus. Thus, PDZ deletion significantly influences the Nterminal conformation of each subunit of HtrA2 trimeric ensemble. Furthermore, structural comparison of $\Delta \Delta \Delta$ with relatively active bacterial counterpart, DegS ${ }^{\Delta \mathrm{PDZ}}$ (PDB ID: 3LGI) demonstrated several significant differences in the regulatory loop movements (RMSD <2.9 $\AA$ ). Although there were no prominent changes in the orientation of the catalytic triad residues, major differences existed in the orientation of loops LA, LD, L2, L3 and their associated secondary structures (Fig. 5D).

Taken together, secondary structural perturbations around activation domain and the regulatory loops seem to play a significant role for decline in $\Delta \Delta \Delta$ enzyme activity. These structural analyses are in concurrence with our functional assays, thereby highlighting the importance of PDZs in restructuring the orientation of regulatory loops near $\Delta \Delta \Delta$ catalytic triad.

\section{DISCUSSION:}


Unlike its homologs, regulation of HtrA2 is multi-layered, plausibly to safeguard untimely proteolysis of its wide array of substrates. Despite numerous efforts, both discrete and collective roles of HtrA2 subunits in promoting the allosteric coordination within the trimeric ensemble remained abstruse till date. Although human HtrAs contains a single PDZ (unlike bacteria), the varying PDZ-mediated regulation suggests an evolutionary diversification of the protease structure across and within species to cater to distinct functional requirements. While various studies emphasize on HtrA2 allostery from a global perspective, the minutiae of the intricate intra- and inter-subunit networking via the regulatory PDZs remain unperceived. Furthermore, a recently published parallel study [28, 29] depicting mathematical model of interdomain interaction raises several questions due to its inability to portray the biological relevance behind experimental interpretations. In this current study, we demonstrate PDZ-mediated synergistic coordination among different domains and subunits of the protease from a biological perspective. Using heterotrimeric HtrA2 variants with varying number of PDZs, we elucidated how individually and/or in unison this domain regulates protease activation. The decline in substrateinduced proteolytic activity of the heterotrimers (WW $\Delta$ and $\mathrm{W} \Delta \Delta$ ) strongly suggests that the contribution of each subunit towards the total residual enzyme activity is defined by the structural identity of both the neighboring subunits, and that the subunits together exist in a cooperatively-shared energy landscape. Interestingly, in contrast to DegS, where the basal activity is inversely proportional to the number of PDZs, our findings demonstrate positive regulation of HtrA2 enzymatic functions by this regulatory domain. Moreover, our work reiterates that sequential removal of PDZs adversely affects thermal stability [17] thus highlighting its role in maintaining structural integrity. 
The structural intricacy in HtrA family has often complicated the understanding of their mechanisms and hence, presence of multiple contradicting reports on their allosteric modulation is not unanticipated. For example, similar to opposing theories on DegS activation [10, 30-32], Chaganti et. al., [17] challenged HtrA2 structural studies [15] and proposed a trans PDZprotease crosstalk-mediated allosteric regulation of HtrA2. The use of activator-cum-substrate $\beta$ casein for the chemical profiling studies assisted in capturing the concerted and synergistic distal binding as well as cleavage mechanism from a more physiological viewpoint. The TAMRAbased active-site modification studies strongly imply the existence of a concerted trans-mediated pathway, wherein absence of one or two PDZs in heterotrimers (mimicking ligand-bound PDZs in cellular milieu) provided an opportunity to the adjacent subunit with intact PDZ(s) to have greater access to the PDZ-lacking subunit, thus allosterically modifying its active-site and preparing it for subsequent substrate binding. The very crowded trimeric ensemble in the apoHtrA2 does not provide much room for such dynamic behavior and hence, might be energetically unfavorable for catalysis. This string of riveting information stimulates a precisely controlled concerted activation mechanism, which might be critical towards participating in several pathways involving a wide repertoire of substrates Similar TAMRA-based studies with heterotrimers lacking one or more active-site serine, along with quantitative enzyme kinetic analyses decidedly substantiate our claim. We have also demonstrated a concerted intermolecular PDZ-protease crosstalk in case of temperature-induced activation of HtrA2. . However, the observed distinct behavior of the heat-based activation might be attributed to the overall dynamism accrued by the PDZs due to rise in temperature. With removal of the PDZ inhibition $(\Delta \Delta \Delta)$, activation via temperature might cause certain favorable rearrangements at the 
N-terminal region [17] and around the active-site such that it compensates for the lost PDZs by opening up the ensemble.

To resolve the ambiguities pertaining to distinct activation pattern in HtrA2 and $\Delta \Delta \Delta$, we reported the structure of catalytically inactive (S306A) version of $\Delta \Delta \Delta$. As the $\Delta \Delta \Delta$ and wild type (PDB ID: 1LCY) structures share similar orientation of catalytic triad residues, we believe that increase in temperature or the binding of specific ligands (activator or substrate) greatly influences the PDZ dynamics and subsequently the active-site reactivity by bringing about suitable regulatory loop rearrangements with respect to the oxyanion hole. For DegS, which is a more open ensemble, and where PDZs act as inhibitory modules, deletion of PDZs do not greatly affect the catalysis of this variant [33-35]. On the contrary, PDZs in HtrA2 seem to play a greater role in structural stabilization by assisting secondary structure formation in protease domains, especially around the regulatory loops that together form the activation domain, which is lost in the $\Delta \Delta \Delta$ variant. Thus, in the absence of any form of activation, PDZs maintain the pyramidal architecture in a closed conformation by stabilizing intramolecular PDZ-protease domain interactions. Absence of inter- and intra-subunit interactions in $\Delta \Delta \Delta$, which could have otherwise favorably assisted establishment of the required transitions (L3 to LD* loop via L1*/L2* (asterisk denotes adjacent subunits)) to the catalytically competent state [16], and also energetically stabilized the protease, reiterates the indispensability of these regulatory domains in upholding the overall protease structural integrity and functions.

Overall, our observations propound a working model for HtrA2 activation, where the initial step of ligand binding or heat activation shifts the intrinsic equilibrium from an inactive state (E) to a higher affinity conformation (E') that eventually promotes a more catalytically competent state 
$\left(\mathrm{E}^{*}\right)$ through a synergistic trans PDZ-protease coordination, as elaborated in Figure 6. Briefly, ligand binding at the exposed substrate binding pocket of PDZ brings about subsequent conformational changes at the PDZ-protease interface that are different from the original inactive state (E). The primary binding leads to displacement of a single PDZ (Es'state), which further commences a synergistic coordination and formation of new interaction networks between the PDZ and protease domains of adjacent subunits, subsequently increasing the affinity of other subunits by opening up the ensemble as well as reorienting the catalytic triad for enhanced catalysis. On the other hand, in temperature-mediated activation, (Fig. 6) the increase in temperature promotes inter-domain PDZ movement in a way that not only reduces the steric crowd but also leads to subtle distal conformational changes conducive toward competent activesite formation. Thus, under cellular conditions, stress-related temperature change might induce initial opening of PDZs, in turn making the catalytic pocket more accessible for substrate binding and proteolysis. Furthermore, this temperature-induced reordering of the active-site ( $\mathrm{E}_{\mathrm{T}}$ 'state) might be particularly important in scenarios where the protease-domain defines initial substrate binding and its subsequent cleavage (unpublished data). Thus, this model describes an energy landscape that might be allosterically tuned in multiple ways to help the protease to conform to different cellular requirements for optimal biological leverage. This kind of dynamic perpetuity, observed in a multimeric protease ensemble through a discrete and sequential inter-domain coupling, allows a robust coalescence of regulatory strategies amongst heterogeneous subensembles that successfully culminate in an amenable functional conformation [36].

In conclusion, this manuscript unequivocally describes the trans-mediated allosteric mechanism of HtrA2 in two different activation modes using a unique retrospective approach through dissecting and rebuilding the protein structure. This study might open up new avenues for 
devising tailor-made strategies toward modulating diverse functions of HtrA2 with desired characteristics for therapeutic benefit. Most importantly, delineation of this multidimensional allosteric model system that amalgamates both activation and stabilization of the macromolecular ensemble through an intricate intermolecular crosstalk for transmission of information within and outside cellular pathways provides an advanced understanding of allostery in general from a more quantitative perspective.

\section{DATA AVAILABILITY}

The newly solved crystal structure reported in this publication is available in RCSB PDB database (http://www.wwpdb.org) under accession number 7VGE (PDB DOI: https://doi.org/10.2210/pdb7VGE/pdb). All the other data are available in the main text or the supplementary materials. Additional data related to this paper may be requested from the authors. 


\section{ACKNOWLEDGEMENTS}

We thank LK. Chaganti, N. Singh and A. Kalarikkal for their intellectual inputs and helpful suggestions related to experimentation. We are grateful to the XRD facility IIT Bombay for initial crystallization screening and crystallographic data collection. This work is supported by the Department of Biotechnology (DBT), Govt. of India (grant number BT/HRD/NWBA/37/01/2015) and intramural research grant received from ACTREC-TMC, India (IEC project no. 162).

\section{AUTHOR CONTRIBUTIONS}

Conceptualization: ALP and KB

Methodology: ALP, VM and PB (structure), SD (in-silico)

Investigation: ALP, VM and PB (structure), SD (in-silico)

Visualization: ALP, VM, SD

Supervision: ALP, PB, KB

Writing - original draft: ALP, SD (in-silico) and KB

Writing — review \& editing: ALP, VM, SD, PB and KB

\section{DECLARATION OF INTERESTS}

The authors declare no competing interests. 


\section{FIGURE LEGENDS}

Figure 1: HtrA2 variants with one or two PDZ domains. (A) Schematic model depicting the denaturation-renaturation strategy that is used to generate heterotrimeric HtrA2 variants (WW $\Delta$ and $\mathrm{W} \Delta \Delta$ ). (B) Native PAGE showing generation of precisely four combinations of HtrA2 variants. Due to significant differences in $\mathrm{pI}$, the electrophoretic separation of the four HtrA2 variant combinations in the renatured reaction mixture (RM) was observed in comparison to the parent homotrimers- wildtype (WWW) and PDZ-deleted construct $(\Delta \Delta \Delta)$. The concentration of the homotrimers used in this analysis was same as that used in the denaturation process to check the presence of any higher order oligomer formation at this concentration. (C) Western Blot analysis confirming the identity of heterotrimers. Native PAGE, as described in (B), was used for transfer of the proteins and subsequent blotting analysis. As the parent $\Delta \Delta \Delta$ construct consisted of an N-terminal FLAG tag, the trimers containing $\Delta$ subunit (W $\Delta \Delta$, WW $\Delta$ and $\Delta \Delta \Delta$ ) were easily probed in the reaction mixture using Anti-FLAG antibody. No band was probed in the WWW lane, while presence of few non-specific bands was observed in $\Delta \Delta \Delta$. The renatured reaction mixture was loaded in diluted form $\left(R M^{D}\right)$ and in the original concentration $\left(R M^{O}\right)$ to confirm the identity of heterotrimers. (D) SDS-PAGE showing the separation of heterotrimeric HtrA2 variants. The variants were separated from the reaction mixture using a very narrow gradient of imidazole in the elution buffer. The separation occurred on the basis of number of histidine residues present in the C-terminal His tag. $\mathrm{W} \Delta \Delta$ eluted in the initial gradients, followed by WWA and WWW. M: Protein marker. (E) Native PAGE showing the separation of heterotrimeric HtrA2 variants. The separated heterotrimeric variants (W $\Delta \Delta$ and $\mathrm{WW} \Delta$ indicated with filled black triangles in their respective lanes) were identified on the basis of their 
electrophoretic mobility with respect to their relative position in the reaction mixture $\left(\mathrm{RM}^{\mathrm{D}}\right.$ and $\left.\mathrm{RM}^{\mathrm{O}}\right)$

Figure 2: Time-course proteolytic cleavage assays of HtrA2 variants. (A) Qualitative gelbased proteolytic cleavage of $\beta$-casein by HtrA2 variants. $2 \mu \mathrm{g}$ of each enzyme variant was incubated individually with $6 \mu \mathrm{g}$ of substrate $\beta$-casein at $37^{\circ} \mathrm{C}$ for different time-points between 0-90 min. The reaction at each time point was stopped with Laemmli buffer at $100{ }^{\circ} \mathrm{C}$. Reaction samples were resolved by $12 \%$ SDS-PAGE and the cleavage pattern was visualized with Coomassie brilliant blue staining. M: Protein marker; EC: Enzyme control; SC: Substrate control. Arrows indicate the cleaved products. (B) Semi quantitative analysis of HtrA2 protease assays. Percentage substrate cleaved at the end of each reaction time point was calculated by quantifying the intensity of residual $\beta$-casein at each time point relative to the uncleaved substrate control (SC) using GelQuant.NET software and was plotted using Graphpad Prism software. WWW displayed maximum substrate cleavage activity within $5 \mathrm{~min}$, followed by $\mathrm{WW} \Delta$ and $\mathrm{W} \Delta \Delta$ that cleaved $90 \%$ substrate by 5 and $10 \mathrm{~min}$ respectively. $\Delta \Delta \Delta$ displayed $100 \%$ substrate cleavage between 30-60 min. The error bars represent the standard errors (SE) calculated from three independent experiments. (C) Quantitative assessment of the HtrA2 proteolytic activity. Initial velocities $\mathrm{V}_{0}$ of HtrA2 and its variants were calculated using FITC labeled- $\beta$ casein as the substrate. The solid lines are the nonlinear least squares fit of the data to the Hill form of the Michaelis-Menten equation: Velocity $=\mathrm{V}_{\max } /\left[1+\left(\mathrm{K}_{0.5} /[\text { substrate }]\right)^{\mathrm{n}}\right]$ using Graphpad Prism software.

Figure 3: Free energy landscape diagrams of HtrA2 variants generated on the basis of catalytic triad orientation. Contour plots for $\beta$-casein bound and temperature-induced (A, B) WWW, (C, D) WW $\Delta(\mathbf{E}, \mathbf{F}) \mathrm{W} \Delta \Delta$ and $(\mathbf{G}, \mathbf{H}) \Delta \Delta \Delta$, respectively, where each contour joined all 
the structures with equal free energies (in $\mathrm{kcal} / \mathrm{mol}$ ). Free energies for all the variants were calculated using the intermolecular distances among the catalytic residues (H198, D228 and S306) as collective variables. The energy gradients have been represented by blue-yellow spectrum where blue represents the lowest and yellow represents the highest free energy.

Figure 4: Trans-mediated allosteric communication under different activation modes. (A) Active-site modification assay performed in presence of substrate. Each variant $(2 \mu \mathrm{M})$ was reacted with TAMRA-FP $(20 \mu \mathrm{M})$ in absence and presence of $\beta$-casein at $37^{\circ} \mathrm{C}$ for $30 \mathrm{~min}$. The reaction mixtures were run on SDS-PAGE, and fluorescence scan of the complete gel was taken using ChemiDoc ${ }^{\mathrm{TM}} \mathrm{MP}$ Imaging System. The fluorescence intensities for the $\mathrm{W}$ and $\Delta$ subunits in each variant were quantified using the Image $\mathrm{Lab}^{\mathrm{TM}}$ software (version 6.0.0 build 25). The values obtained for each subunit from multiple independent experiments were averaged and the fold changes between the tests with respect to their corresponding controls were plotted using Graphpad Prism software. (B) Active-site modification assay performed at increased temperature. Each variant $(2 \mu \mathrm{M})$ was reacted with TAMRA-FP $(20 \mu \mathrm{M})$ and incubated either at $37{ }^{\circ} \mathrm{C}$ (Control) or at $50{ }^{\circ} \mathrm{C}$ (Test) for $30 \mathrm{~min}$. The fluorescence imaging analysis and quantification were done similarly as in (A). (C) Quantitative assessment of proteolytic activity of HtrA2 variants with active-site mutation. Initial velocities $\mathrm{V}_{0}$ of HtrA2 and its variants were calculated using FITC labeled- $\beta$ casein as the substrate. The solid lines are the nonlinear least squares fit of the data to the Hill form of the Michaelis-Menten equation: Velocity $=\mathrm{V}_{\max } /[1+$ $\left.\left(\mathrm{K}_{0.5} /[\text { substrate }]\right)^{\mathrm{n}}\right]$ using Graphpad Prism software. (D) Model representing the trans-mediated allosteric communication between the adjacent subunits of HtrA2 trimeric ensemble.

Figure 5: Structural features of inactive $\operatorname{HtrA2} \Delta \Delta \Delta$ variant (PDB ID: 7VGE). (A) Surface representation of the two $\Delta \Delta \Delta$ trimers (ABC and DEF) obtained in the asymmetric unit. Left 
panel: Top-view of the association, with the N-terminus of one trimer (DEF) pointing outwards. Right panel: Side-view of the association, with the two trimers having their respective Nterminus pointing in opposite directions. Chains A, B, C, D, E and F are represented in pale green, pale blue, pink, pale yellow, mauve and grey respectively. (B) Cross-section view showing the arrangement of LA loop and the corresponding $\beta 1-\beta 2$ between two alternate chains. In the absence of C-terminal PDZ domain, a single trimer (ABC) appears to stabilize itself by associating with another similar trimer (DEF) via the LA loop. (C) Superposition of full-length HtrA2 (PDB ID: 1LCY - light blue) with $\Delta \Delta \Delta$ (pale yellow). Left panel: Orientation of LA loop of 1LCY (salmon) and $\Delta \Delta \Delta$ (Chain D) shows the absence of secondary structure for $\beta 1-\beta 2$ in $\Delta \Delta \Delta$. Middle panel: $\beta 7-\beta 8$ followed by LD loop (orange in 1LCY) forms an extended secondary structure in $\Delta \Delta \Delta$. Right panel: Absence of $\alpha 5$ helix (green cyan) of the PDZ domain might result in a partial loss of secondary structure of $\beta 11-\beta 12$, and absence of L2 loop (deep olive in 1LCY) in $\Delta \Delta \Delta$ variant. (D) Superposition of DegS ${ }^{\Delta P D Z}$ (PDB ID: 3LGI - green cyan) with $\Delta \Delta \Delta$ (Chain D - pale yellow; Chain A - pale green). Conformational changes in 3LGI and $\Delta \Delta \Delta$ are depicted in orange and green, respectively. Left panel: The LD loop shows minor conformational changes, while the L3 loop is completely missing in $\Delta \Delta \Delta$ (chain D). Middle panel: The LA loop displays significant difference in its orientation in 3LGI and $\Delta \Delta \Delta$ (chain D). Right panel: Differences in orientation of L2 loop in 3LGI and $\Delta \Delta \Delta$ (chain A). All the images were generated using PyMOL Version 1.3 (Schrodinger, LLC).

Figure 6: Model depicting different modes of HtrA2 activation. HtrA2 in the basal state exhibits very little activity, as the PDZs guard the entrance of the pyramidal ensemble and restrict the substrate entry (step Ia). Initial binding of the ligand at the exposed allosteric pocket of the PDZ of the original inactive state (E) induces subsequent conformational changes at the 
PDZ-protease domain interface (Es') (step II). On the contrary, in case of activation via heat shock (step Ib), the exposed PDZs of the mature inactive protease experience increased thermal motion and induce favorable conformational changes around the active-sites in the adjacent subunits ( $\left.\mathrm{E}_{\mathrm{T}}{ }^{\prime}\right)$. The primary binding or activation event leads to displacement of a single PDZ, which further commences the synergistic coordination and formation of new interaction networks between the PDZ and protease domains of adjacent subunits (Es' or $\mathrm{E}_{\mathrm{T}}$ '), subsequently increasing the affinity of other subunits towards the substrate by opening up the ensemble (step III) including the $\mathrm{N}$-terminal region as well as rearranging the catalytic triad orientation ( $\left.\mathrm{E}^{*}\right)$ for enhanced catalysis. 


\section{TABLES}

Table 1: Steady state enzyme kinetic parameters for HtrA2 variants. The initial rates for the cleavage of FITC labeled- $\beta$ casein by different HtrA2 variants were measured and fitted to the Hill form of the Michaelis-Menten equation to determine the steady-state kinetic parameters (Figs 2 and S2). Values are the mean \pm SEM and are generated from data points obtained from at least three independent experiments.

\begin{tabular}{|c|c|c|c|c|c|}
\hline $\begin{array}{c}\text { HtrA2 } \\
\text { Variants }\end{array}$ & $\begin{array}{c}V_{\max }\left(\mathrm{Ms}^{-1}\right) \\
\times 10^{-10}\end{array}$ & $\mathbf{K}_{0.5}(\boldsymbol{\mu M})$ & $\begin{array}{r}k_{\text {cat }}\left(\mathrm{s}^{-1}\right) \\
\text { X } 10^{-05}\end{array}$ & $k_{c a t} / K_{m}\left(M^{-1} s^{-1}\right)$ & Hill constant \\
\hline WWW & $2.892 \pm 0.0002$ & $2.513 \pm 0.2$ & 14.46 & 57.534 & $2.357 \pm 0.3$ \\
\hline WW & $1.360 \pm 0.0007$ & $1.738 \pm 0.4$ & 6.81 & 39.154 & $1.231 \pm 0.3$ \\
\hline $\mathbf{W} \Delta \boldsymbol{\Delta}$ & $0.713 \pm 0.0001$ & $1.293 \pm 0.1$ & 3.57 & 27.578 & $1.272 \pm 0.1$ \\
\hline$\Delta \mathbf{\Delta} \boldsymbol{\Delta}$ & $0.289 \pm 0.0001$ & $0.869 \pm 0.1$ & 1.44 & 16.607 & $1.367 \pm 0.3$ \\
\hline $\mathrm{W}_{\mathrm{I}} \Delta \mathbf{\Delta}$ & $0.569 \pm 0.00008$ & $0.931 \pm 0.1$ & 2.84 & 30.546 & $1.824 \pm 0.2$ \\
\hline $\mathbf{W}_{\mathrm{I}} \mathbf{W}_{\mathrm{I}} \Delta$ & $1.219 \pm 0.0003$ & $1.771 \pm 0.2$ & 6.09 & 34.401 & $2.353 \pm 0.5$ \\
\hline $\mathbf{W} \Delta_{I} \Delta_{I}$ & $0.157 \pm 0.00004$ & $0.423 \pm 0.1$ & 0.78 & 18.504 & $2.714 \pm 0.9$ \\
\hline$\overline{W W} \Delta_{I}$ & $0.412 \pm 0.0002$ & $0.877 \pm 0.2$ & 2.11 & 24.022 & $2.077 \pm 0.8$ \\
\hline
\end{tabular}




\section{STAR METHODS:}

\section{Recombinant protein expression and purification}

The mature form of HtrA2 full length protein, comprising residues 134-458, is considered as Wildtype (WT or WWW) and the PDZ lacking HtrA2 variant containing the N-terminus-SPD domain (residues 134-210) is considered as N-SPD or $\Delta \Delta \Delta$. These proteins are cloned and expressed in pET-20b (Addgene, Cambridge, MA, USA) and modified pMAL-c5E vectors (New England Biolabs, Ipswich, MA, USA) respectively, as previously described [17]. The latter had a TEV (Tobacco Etch Virus) protease cleavage site introduced for removal of the fusion tag. An additional FLAG octapeptide (DYKDDDDK) tag at the N-terminal end in $\Delta \Delta \Delta$ construct and single amino acid substitution of S306 residue to Alanine in both the constructs (WWW and $\Delta \Delta \Delta$ ) was performed using site-directed mutagenesis (Stratagene, Austin, TX, USA). The insertion and point mutations were verified by automated DNA sequencing. Recombinant proteins were expressed in E. coli BL21 (DE3) pLysS and Rosetta (DE3) pLysS strains (Novagen, Billerica, MA, USA). These cells were grown at $37^{\circ} \mathrm{C}$ until the optical density at 600 $\mathrm{nm}$ reached $\sim 0.6 \mathrm{AU}$ and the protein expression was then induced by adding isopropyl $\beta$-D-1thiogalactopyranoside (IPTG) to a final concentration of $0.3 \mathrm{mM}$. The cells were further cultured at $18{ }^{\circ} \mathrm{C}$ for $18 \mathrm{~h}$ post induction and then harvested for protein production. Protein variants with a C-terminal Histidine tag were purified by affinity chromatography using Ni-NTA resin (Nucleo-pore, Genetix Biotech Asia Pvt. Ltd), while those with N-terminal maltose binding protein (MBP) fusion tag were purified using amylose resin (New England Biolabs). All the protein variants were purified in buffer A (20 mM Na $2 \mathrm{HPO}_{4} / \mathrm{NaH}_{2} \mathrm{PO}_{4}(\mathrm{pH}$ 8.0) containing 100 $\mathrm{mM} \mathrm{NaCl}$ and 3\% (v/v) glycerol) and the proteins obtained were $>95 \%$ pure, as estimated by SDS PAGE. 


\section{Generation and purification of heterotrimeric HtrA2 variants}

For obtaining heterotrimeric variants, the use of simultaneous heat and chemical denaturation process was employed. Purified WWW and $\Delta \Delta \Delta$ trimers were mixed in equimolar concentrations and diluted up to 15-fold in buffer A, additionally containing $6 \mathrm{M}$ guanidinium chloride ( $\mathrm{pH}$ 8.0). This reaction mixture was further subjected to heat by incubating at $50{ }^{\circ} \mathrm{C}$ for 1-2 h for efficient denaturation into monomeric subunits. The solution was further dialyzed extensively against a renaturation buffer (buffer A (pH 8.0) containing $300 \mathrm{mM}$ arginine with varying content of glycerol) at $4{ }^{\circ} \mathrm{C}$. The final step involved dialysis in buffer A (to remove arginine) and concentrating the renatured protein mixture using an Ultracel-30k centrifugal filter (Merck Millipore). For further separation of the different HtrA2 variants (homo- and heterotrimers), this renatured mixture was bound to Ni-IDA column pre-equilibrated with buffer A. Since the variants differed in the total number of histidine residues (WWW-His6 and $\Delta \Delta \Delta$-His 3 ) at their C-terminal end, a very shallow gradient of imidazole (10-250 mM) in the elution buffers was used for their separation. The impurities eluted in the flow-through fraction and up to 30 $\mathrm{mM}$ imidazole buffer washes, followed by $\mathrm{W} \Delta \Delta, \mathrm{WW} \Delta$ and WWW eluting between $30-250$ $\mathrm{mM}$ imidazole containing elution buffers. The elution fractions were loaded on SDS PAGE for analyzing the fractions containing the heterotrimers.

\section{Native PAGE and Western Blot}

To confirm the identity of the generated HtrA2 heterotrimers, protein samples from the appropriate purification fractions were mixed with native PAGE sample buffer (62.5 mM Tris$\mathrm{Cl}$, pH 6.8, 15\% glycerol, and $0.01 \%$ bromophenol blue) and loaded on $7.5 \%$ native PAGE. The gels were run at a constant voltage of $50 \mathrm{~V}$ for $5 \mathrm{~h}$ at $4{ }^{\circ} \mathrm{C}$ and were analyzed by Coomassie Blue 
staining. Since all the homo- and hetero-trimers also differed in the number of FLAG tag (originally introduced in $\Delta \Delta \Delta$ construct), the unstained native PAGE gel was additionally probed with monoclonal anti-FLAG antibody by performing Western blot. The separated protein bands were transferred on nitrocellulose membrane (Millipore, Italy) using Invitrogen wet transfer mini blot module (Thermo Fisher Scientific, USA) in 1X transfer buffer (25 mM Tris, $192 \mathrm{mM}$ glycine, $20 \%(\mathrm{v} / \mathrm{v})$ methanol, $0.025-0.1 \%$ SDS, $\mathrm{pH}$ 8.3) at a constant voltage of $15 \mathrm{~V}$ for $60-90$ min. The membrane was then blocked with $5 \%$ bovine serum albumin (BSA) prepared in $1 \mathrm{X}$ TBST buffer (Tris-buffered saline (TBS) containing $0.1 \%$ Tween-20) for $1 \mathrm{~h}$, followed by overnight incubation with 1:2000 dilution of anti-FLAG antibody (Sigma Aldrich) at $4{ }^{\circ} \mathrm{C}$. The membrane was further incubated at room temperature with 1:5000 dilution of goat antimouse IgG HRPO (Sigma Aldrich) secondary antibody for $1 \mathrm{~h}$. After each incubation period mentioned above, the membrane was washed three to six times with $1 \mathrm{X}$ TBST solution. Antibody dilutions were prepared in TBS containing 0.01\% Tween-20 and 1\% BSA. Bands were developed using an enhanced chemiluminescence kit (Thermo Fischer, USA).

\section{Protease assays}

Protease activity of different HtrA2 variants (homo- and hetero-trimers) was semi-quantitatively determined using the generic serine protease substrate $\beta$-casein (Sigma, St. Louis, MO, U.S.A.). For each $30 \mu \mathrm{l}$ reaction, $2 \mu \mathrm{g}$ of respective protein variant was incubated with $6 \mu \mathrm{g}$ of $\beta$-casein in buffer $\mathrm{A}$ at $37^{\circ} \mathrm{C}$ for a course of different time points (0-90 min). The reaction was stopped with a 5X SDS sample loading buffer, followed by boiling for $10 \mathrm{~min}$. The cleavage products were resolved on 15\% SDS PAGE and the results were analyzed by Coomassie staining. Protein bands were quantitated using GelQuant.NET software. The data was normalized with respect to the uncleaved substrate control and was plotted using the GraphPad Prism 5 (GraphPad Software, 
San Diego, CA, USA). For all quantitative studies, fluorescein isothiocyanate (FITC) $\beta$-casein (Sigma, St. Louis, MO, U.S.A.) was used as the substrate. For each $100 \mu$ l reaction mixture, 2 $\mu \mathrm{M}$ protein was incubated with increasing concentrations $(0-20 \mu \mathrm{M})$ of FITC $\beta$-casein at $37^{\circ} \mathrm{C}$ in buffer A. The rate of proteolytic cleavage was monitored in Cytation ${ }^{\mathrm{TM}} 5$ multi-mode plate reader (BioTek Instruments, Inc.) using an excitation wavelength of $485 \mathrm{~nm}$, followed by emission at $535 \mathrm{~nm}$. For each HtrA2 variant, the initial velocities $\mathrm{V}_{0}(\mu \mathrm{M} / \mathrm{min})$ were calculated using linear regression analysis. The steady-state kinetic parameters were calculated from the reaction rates by fitting the data to the Hill form of Michaelis-Menten equation, Velocity = $\mathrm{V}_{\max } /\left[1+\left(\mathrm{K}_{0.5} /[\text { substrate }]\right)^{\mathrm{n}}\right]$ (where $\mathrm{V}_{\max }$ represents the maximum velocity and $\mathrm{K}_{0.5}$ is the substrate concentration at half maximal velocity) in GraphPad Prism version 5.01 for Windows (GraphPad Software, San Diego, California USA). All the experiments were performed independently in triplicates and the mean SEM values were reported.

\section{Activation assay using chemical probe}

For trapping the active-site reactivity, chemical profiling of the enzyme activity was performed using ActivX TAMRA-FP Serine Hydrolase Probe (Thermo Scientific, USA). 0.1mM stock of TAMRA-FP was prepared by dissolving the commercially available probe in anhydrous dimethyl sulfoxide, according to the manufacturer's protocol. For each reaction mixture, a final concentration of $2 \mu \mathrm{M}$ probe was added and the reactions were incubated for $30 \mathrm{~min}$. For substrate-based and temperature-induced activation, the incubation temperatures were set to 37 ${ }^{\circ} \mathrm{C}$ and $50{ }^{\circ} \mathrm{C}$ respectively. The reactions were terminated by adding 5X SDS sample loading buffer, followed by boiling for $10 \mathrm{~min}$. The labeled proteins were resolved on 15\% SDS PAGE and the results were analyzed using ChemiDoc ${ }^{\mathrm{TM}}$ MP Imaging System (Bio-Rad laboratories, Inc.) with $C y \circledast 3$ filters. For better quantitative analysis, the fluorescence intensities of each 
subunit band were measured using Image $\mathrm{Lab}^{\mathrm{TM}}$ - version 6.0.0 build 25 (2017, Bio-Rad laboratories, Inc.) and the fold change between the intensities of the subunits in the test samples with respect to their corresponding controls were then plotted for graphical representation.

\section{In silico preparation of the heterotrimeric models of HtrA2}

For preparation of in silico heterotrimeric models, three-dimensional (3D) crystal structure of HtrA2 (PDB ID: 5M3N) was retrieved from Protein Data Bank [37]. The 3D structure lacked crystal information for few loop regions- an N-terminal loop $\left({ }^{1}\right.$ AVPSPPPA $\left.{ }^{8}\right)$, a loop in the SPD region $\left({ }^{280} \mathrm{RPARDLGLPQTNV}{ }^{292}\right)$ and the linker region $\left({ }^{344} \mathrm{RGEKKNSSSGISGS}{ }^{357}\right)$ [37]. Hence, these missing regions were modeled using $a b$ initio mode of loop filling and protein preparation programs in Prime (Prime, Schrödinger, LLC, New York, NY, 2020) [38]. Loop filling was done on the basis of permissive dihedral angle values for different residues, followed by repetitive rounds of sample loop clustering, optimizing the side-chain, and energy minimization of the loops [38]. The resultant loop-filled wildtype HtrA2 monomer was energy minimized using Desmond (Desmond, Schrödinger, LLC, New York, NY, 2020). Using the crystal symmetry data of HtrA2 monomer [37], the trimeric ensemble of HtrA2 wildtype (WWW) was generated, which was further modified by deletion of the linker and PDZ domain in subsequent chains, viz, WW $\Delta$ (with linker and PDZ deleted in chain C), W $\Delta \Delta$ (with linker and PDZ deleted in chain B and C), and, $\Delta \Delta \Delta$ (with linker and PDZ deleted in all the three chains). These models were then subjected to docking using a $\beta$-casein peptide denoted by GPFPIIV (a generic substrate). Docking was performed using Bioluminate software (Bioluminate, Schrödinger, LLC, New York, NY, 2020) and scored using MM-GBSA (Molecular Mechanics using Generalized Born and Surface Area continuum) scoring method [39]. The top five docked 
complexes for $\beta$-casein-bound HtrA2 variants were analysed for the identification of interacting surface residues using PDBSum online server [40].

\section{Molecular dynamic simulation (MDS) analysis of bound and unbound HtrA2 variants}

Both peptide-bound and unbound forms of WWW, WW $\Delta, \mathrm{W} \Delta \Delta$ and $\Delta \Delta \Delta$ were subjected to MDS using Desmond (Desmond, Schrödinger, LLC, New York, NY, 2020) where OPLS3e (Optimized Potentials for Liquid Simulations Version 3e) force field was used to generate topology and parameter files [41, 42]. Each protein structure was surrounded by a cubic box of TIP3P water molecules with the nearest distance from the protein to the box boundary being no more than $10 \AA$ [43]. The generated systems were subsequently neutralized (net charge was brought to zero) by adding adequate number of positive $\left(\mathrm{Na}^{+}\right)$ions. Each system underwent one round of steepest- descent minimization, followed by one round of conjugated gradient for 5000 picosecond (ps) [44]. The systems were then equilibrated in NVT (constant number of particles, volume, and temperature) and NPT (constant number of particles, pressure, and temperature) ensembles with two sets of restrained NVT (for 24 ps and 2000 ps respectively) and one set of restrained (for 24 ps) and unrestrained (for 5000 ps) NPT each [45]. During equilibration, LINCS (LINear Constraint Solver) constraint algorithm was used to apply position restraining force on all the atomic bonds present in the systems [46]. All the systems were then subjected to final MD simulation run for 1000 nanosecond (ns) under no-restrained NPT ensemble. For peptide-bound systems, the final production runs were performed at $300 \mathrm{~K}$. However, for analyzing the temperature dependent changes in the unbound systems, the final temperature was kept at $323 \mathrm{~K}$. Post-simulation these systems were further subjected to meta-dynamics analysis for 50 ns that generated free energy landscape data on the basis of the active-site conformation. All the MD 
simulation data analysis was carried using MD simulation analysis tools available in Desmond and Maestro platform (Desmond and Maestro, Schrödinger, LLC, New York, NY, 2020).

\section{Protein crystallization}

To eliminate the possibility of partial protein degradation at higher concentrations required for crystallization, the inactive variant of $\mathrm{N}-\mathrm{SPD}(\Delta \Delta \Delta-\mathrm{S} 306 \mathrm{~A})$ was used. The protein was purified in buffer containing 10 mM HEPES (pH 8.0), $100 \mathrm{mM} \mathrm{NaCl}$, and 3\% glycerol, in a similar way as described for active $\Delta \Delta \Delta$. Four commercial crystallization screens from Hampton Research (HR2-110 and HR2-112) and Molecular Dimensions (Structure Screens MD1 -01 and MD1 -02) were used for setting crystallization trials using protein sample with concentration of $15 \mathrm{mg} / \mathrm{ml}$. The optimization screen was set using hanging drop vapor diffusion after mixing protein ( 6 $\mathrm{mg} / \mathrm{mL}$ ) in 1:1 ratio with mother liquor comprising $0.5 \mathrm{M}$ Sodium acetate trihydrate, $2.0 \mathrm{M}$ Sodium formate $\mathrm{pH}$ 6.0, 3\% glycerol, and diffraction-quality crystals were obtained after two weeks at $16{ }^{\circ} \mathrm{C}$.

\section{Data collection and structure determination}

Prior to diffraction data collection, the crystals were soaked in a cryo-protectant solution (mother liquor with $20 \%$ ethylene glycol) and then flash frozen in a liquid nitrogen stream at $100 \mathrm{~K}$. Diffraction data were collected by the rotation method with a Rigaku MicroMax 007HF generator equipped with R-Axis IV++ detector using $\mathrm{CuK} \alpha \mathrm{X}$-ray radiation (1.5418 $\AA$ ) at the Protein Crystallography Facility, IIT Bombay, Mumbai. Diffraction data was collected with an exposure of $10 \mathrm{~min}$, at a detector distance of $370 \mathrm{~mm}$. The crystal was subjected to sequential steps of annealing (of $5 \mathrm{~s}$ each) for a total of $15 \mathrm{~s}$. The diffraction images were indexed, integrated, and scaled with XDS [47]. The intensities were converted to the structure factors with 
the programs F2MTZ and CAD of CCP4 [48]. The data collection statistics are presented in Table S4. The calculated Matthew's coefficient for $\Delta \Delta \Delta$ is $2.49 \AA^{3} \mathrm{Da}^{-1}$ that indicated the presence of six molecules in the asymmetric unit. The phases were obtained by molecular replacement with the program PHASER [49] using the coordinates of the N-terminal region and protease domain of the previously reported structure (PDB ID: ILCY). Further rounds of manual model building and electron density interpretation was performed in COOT [50] and refinement was executed with REFMAC5 [51]. The first few N-terminal residues in an asymmetric unit and the flexible loops of certain regions could not be modeled due to the lack of proper electron density. The final refinement statistics and validation parameters obtained with MolProbity [52] of the refined structure are summarized in Table S4. All structure-related figures were generated with PyMOL (https://www.pymol.org/). 


\section{REFERENCES}

1. Traut, T., Enzyme Activity: Allosteric Regulation. eLS, 2014.

2. $\quad$ Ma, B., et al., Dynamic Allostery: Linkers Are Not Merely Flexible. Structure, 2011. 19(7): p. 907-917.

3. Basu, M.K., E. Poliakov, and I.B. Rogozin, Domain mobility in proteins: functional and evolutionary implications. Briefings in bioinformatics, 2009. 10(3): p. 205-216.

4. Bagowski, C.P., W. Bruins, and A.J.W. Te Velthuis, The nature of protein domain evolution: shaping the interaction network. Current genomics, 2010. 11(5): p. 368-376.

5. Kim, E. and M. Sheng, PDZ domain proteins of synapses. Nature Reviews Neuroscience, 2004. 5(10): p. 771-781.

6. Hung, A.Y. and M. Sheng, PDZ Domains: Structural Modules for Protein Complex Assembly*. Journal of Biological Chemistry, 2002. 277(8): p. 5699-5702.

7. Lee, H.-J. and J.J. Zheng, PDZ domains and their binding partners: structure, specificity, and modification. Cell Communication and Signaling, 2010. 8(1): p. 8.

8. Joanna, S.-G., et al., HtrA Protease Family as Therapeutic Targets. Current Pharmaceutical Design, 2013. 19(6): p. 977-1009.

9. Singh, N., R.R. Kuppili, and K. Bose, The structural basis of mode of activation and functional diversity: A case study with HtrA family of serine proteases. Archives of Biochemistry and Biophysics, 2011. 516(2): p. 85-96.

10. Clausen, T., et al., HTRA proteases: regulated proteolysis in protein quality control. Nature Reviews Molecular Cell Biology, 2011. 12(3): p. 152-162.

11. Vierkotten, S., P.S. Muether, and S. Fauser, Overexpression of HTRA1 Leads to Ultrastructural Changes in the Elastic Layer of Bruch's Membrane via Cleavage of Extracellular Matrix Components. PLOS ONE, 2011. 6(8): p. e22959.

12. Yang, Q.-H., et al., Omi/HtrA2 catalytic cleavage of inhibitor of apoptosis (IAP) irreversibly inactivates IAPs and facilitates caspase activity in apoptosis. Genes \& development, 2003. 17(12): p. 1487-1496.

13. Suzuki, Y., et al., A Serine Protease, HtrA2, Is Released from the Mitochondria and Interacts with XIAP, Inducing Cell Death. Molecular Cell, 2001. 8(3): p. 613-621.

14. Suzuki, Y., et al., X-linked Inhibitor of Apoptosis Protein (XIAP) Inhibits Caspase-3 and -7 in Distinct Modes *. Journal of Biological Chemistry, 2001. 276(29): p. 27058-27063.

15. Li, W., et al., Structural insights into the pro-apoptotic function of mitochondrial serine protease HtrA2/Omi. Nature Structural Biology, 2002. 9(6): p. 436-441.

16. Bejugam, P.R., et al., Allosteric Regulation of Serine Protease HtrA2 through Novel NonCanonical Substrate Binding Pocket. PLOS ONE, 2013. 8(2): p. e55416.

17. Chaganti, L.K., R.R. Kuppili, and K. Bose, Intricate structural coordination and domain plasticity regulate activity of serine protease HtrA2. FASEB J, 2013. 27(8): p. 3054-66.

18. Gieldon, A., et al., Distinct 3D Architecture and Dynamics of the Human HtrA2(Omi) Protease and Its Mutated Variants. PLOS ONE, 2016. 11(8): p. e0161526.

19. Jarzab, M., et al., Intra- and intersubunit changes accompanying thermal activation of the HtrA2(Omi) protease homotrimer. Biochimica et Biophysica Acta (BBA) - Proteins and Proteomics, 2016. 1864(3): p. 283-296.

20. Martins, L.M., et al., Binding Specificity and Regulation of the Serine Protease and PDZ Domains of HtrA2/Omi*. Journal of Biological Chemistry, 2003. 278(49): p. 4941749427. 
21. Spiess, C., A. Beil, and M. Ehrmann, A Temperature-Dependent Switch from Chaperone to Protease in a Widely Conserved Heat Shock Protein. Cell, 1999. 97(3): p. 339-347.

22. Singh, N., et al., Dual regulatory switch confers tighter control on HtrA2 proteolytic activity. The FEBS Journal, 2014. 281(10): p. 2456-2470.

23. Merski, M., et al., Molecular motion regulates the activity of the Mitochondrial Serine Protease HtrA2. Cell Death \& Disease, 2017. 8(10): p. e3119-e3119.

24. Liu, Y., M.P. Patricelli, and B.F. Cravatt, Activity-based protein profiling: The serine hydrolases. Proceedings of the National Academy of Sciences, 1999. 96(26): p. 14694.

25. Gasteiger, E., et al., Protein Identification and Analysis Tools on the ExPASy Server, in The Proteomics Protocols Handbook, J.M. Walker, Editor. 2005, Humana Press: Totowa, NJ. p. 571-607.

26. Martins, L.M., et al., Binding Specificity and Regulation of the Serine Protease and PDZ Domains of HtrA2/Omi. Journal of Biological Chemistry, 2003. 278(49): p. 4941749427.

27. Bussi, G. and A. Laio, Using metadynamics to explore complex free-energy landscapes. Nature Reviews Physics, 2020. 2(4): p. 200-212.

28. Toyama, Y., R.W. Harkness, and L.E. Kay, Dissecting the role of interprotomer cooperativity in the activation of oligomeric high-temperature requirement A2 protein. Proceedings of the National Academy of Sciences, 2021. 118(35): p. e2111257118.

29. Toyama, Y., et al., Oligomeric assembly regulating mitochondrial HtrA2 function as examined by methyl-TROSY NMR. Proceedings of the National Academy of Sciences, 2021. 118(11): p. e2025022118.

30. Hasselblatt, H., et al., Regulation of the sigmaE stress response by DegS: how the PDZ domain keeps the protease inactive in the resting state and allows integration of different OMP-derived stress signals upon folding stress. Genes \& development, 2007. 21(20): p. 2659-2670.

31. Mauldin, R.V. and R.T. Sauer, Allosteric regulation of DegS protease subunits through a shared energy landscape. Nature Chemical Biology, 2013. 9(2): p. 90-96.

32. Wilken, C., et al., Crystal Structure of the DegS Stress Sensor: How a PDZ Domain Recognizes Misfolded Protein and Activates a Protease. Cell, 2004. 117(4): p. 483-494.

33. Walsh, N.P., et al., OMP Peptide Signals Initiate the Envelope-Stress Response by Activating DegS Protease via Relief of Inhibition Mediated by Its PDZ Domain. Cell, 2003. 113(1): p. 61-71.

34. Sohn, J., R.A. Grant, and R.T. Sauer, Allosteric Activation of DegS, a Stress Sensor PDZ Protease. Cell, 2007. 131(3): p. 572-583.

35. Sohn, J., R.A. Grant, and R.T. Sauer, Allostery is an intrinsic property of the protease domain of DegS: implications for enzyme function and evolution. The Journal of biological chemistry, 2010. 285(44): p. 34039-34047.

36. Motlagh, H.N., et al., The ensemble nature of allostery. Nature, 2014. 508(7496): p. 331339.

37. Merski, M., et al., Molecular motion regulates the activity of the Mitochondrial Serine Protease HtrA2. Cell death \& disease, 2017. 8(10): p. e3119-e3119.

38. Jacobson, M.P., et al., A hierarchical approach to all-atom protein loop prediction. Proteins: Structure, Function, and Bioinformatics, 2004. 55(2): p. 351-367.

39. Hou, T., et al., Assessing the performance of the molecular mechanics/Poisson Boltzmann surface area and molecular mechanics/generalized Born surface area methods. II. The 
accuracy of ranking poses generated from docking. Journal of computational chemistry, 2011. 32(5): p. 866-877.

40. Laskowski, R.A., et al., PDBsum: Structural summaries of PDB entries. Protein science : a publication of the Protein Society, 2018. 27(1): p. 129-134.

41. Jorgensen, W.L., D.S. Maxwell, and J. Tirado-Rives, Development and Testing of the OPLS All-Atom Force Field on Conformational Energetics and Properties of Organic Liquids. Journal of the American Chemical Society, 1996. 118(45): p. 11225-11236.

42. Harder, E., et al., OPLS3: A Force Field Providing Broad Coverage of Drug-like Small Molecules and Proteins. Journal of Chemical Theory and Computation, 2016. 12(1): p. 281-296.

43. Mark, P. and L. Nilsson, Structure and Dynamics of the TIP3P, SPC, and SPC/E Water Models at 298 K. The Journal of Physical Chemistry A, 2001. 105(43): p. 9954-9960.

44. Wardi, Y., A stochastic steepest-descent algorithm. Journal of Optimization Theory and Applications, 1988. 59(2): p. 307-323.

45. McDonald, I.R., NpT-ensemble Monte Carlo calculations for binary liquid mixtures. Molecular Physics, 1972. 23(1): p. 41-58.

46. Hess, B., et al., LINCS: A linear constraint solver for molecular simulations. Journal of Computational Chemistry, 1997. 18(12): p. 1463-1472.

47. Kabsch, W., XDS. Acta crystallographica. Section D, Biological crystallography, 2010. 66(Pt 2): p. 125-132.

48. Winn, M.D., et al., Overview of the CCP4 suite and current developments. Acta crystallographica. Section D, Biological crystallography, 2011. 67(Pt 4): p. 235-242.

49. McCoy, A.J., et al., Phaser crystallographic software. Journal of applied crystallography, 2007. 40(Pt 4): p. 658-674.

50. Emsley, P. and K. Cowtan, Coot: model-building tools for molecular graphics. Acta crystallographica. Section D, Biological crystallography, 2004. 60(Pt 12 Pt 1): p. 21262132.

51. Murshudov, G.N., et al., REFMAC5 for the refinement of macromolecular crystal structures. Acta crystallographica. Section D, Biological crystallography, 2011. 67(Pt 4): p. 355-367.

52. Davis, I.W., et al., MolProbity: structure validation and all-atom contact analysis for nucleic acids and their complexes. Nucleic Acids Research, 2004. 32(suppl_2): p. W615W619. 
A

HtrA2 WT

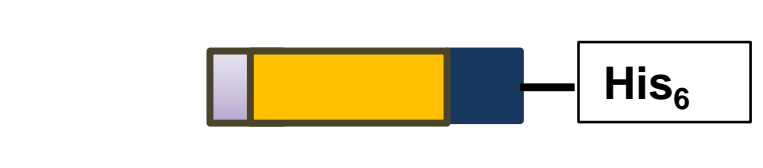

MBP $\stackrel{\text { TEV site }}{\downarrow}$ FLAG
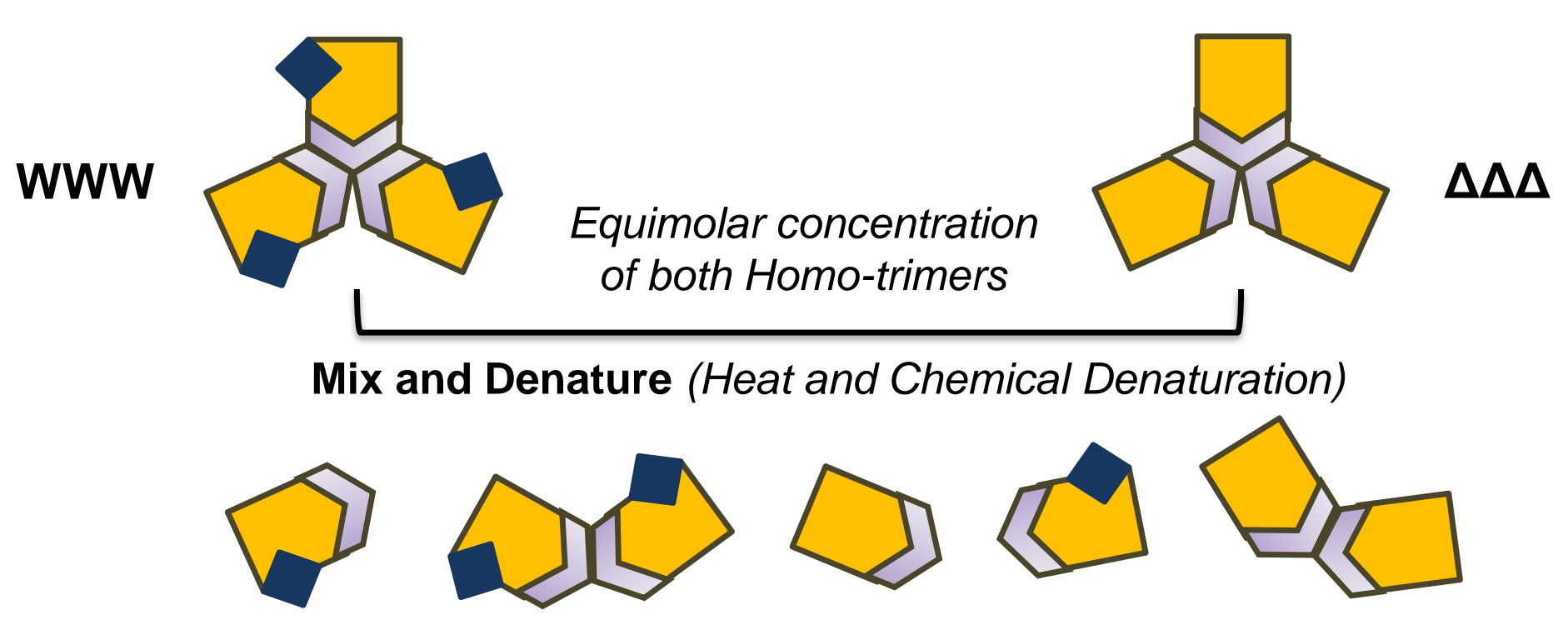

Renature (Refolding through Dialysis)
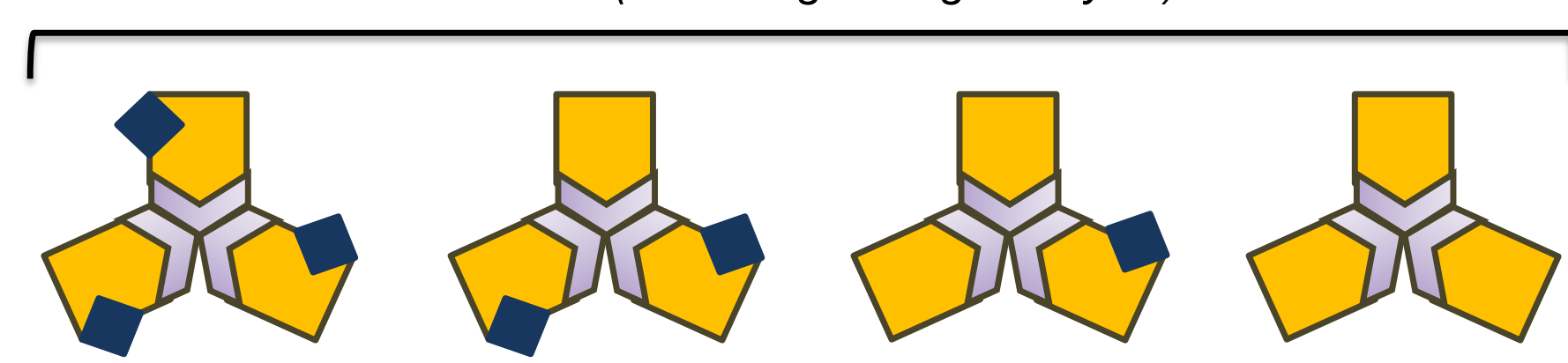

WWW
WWA
B

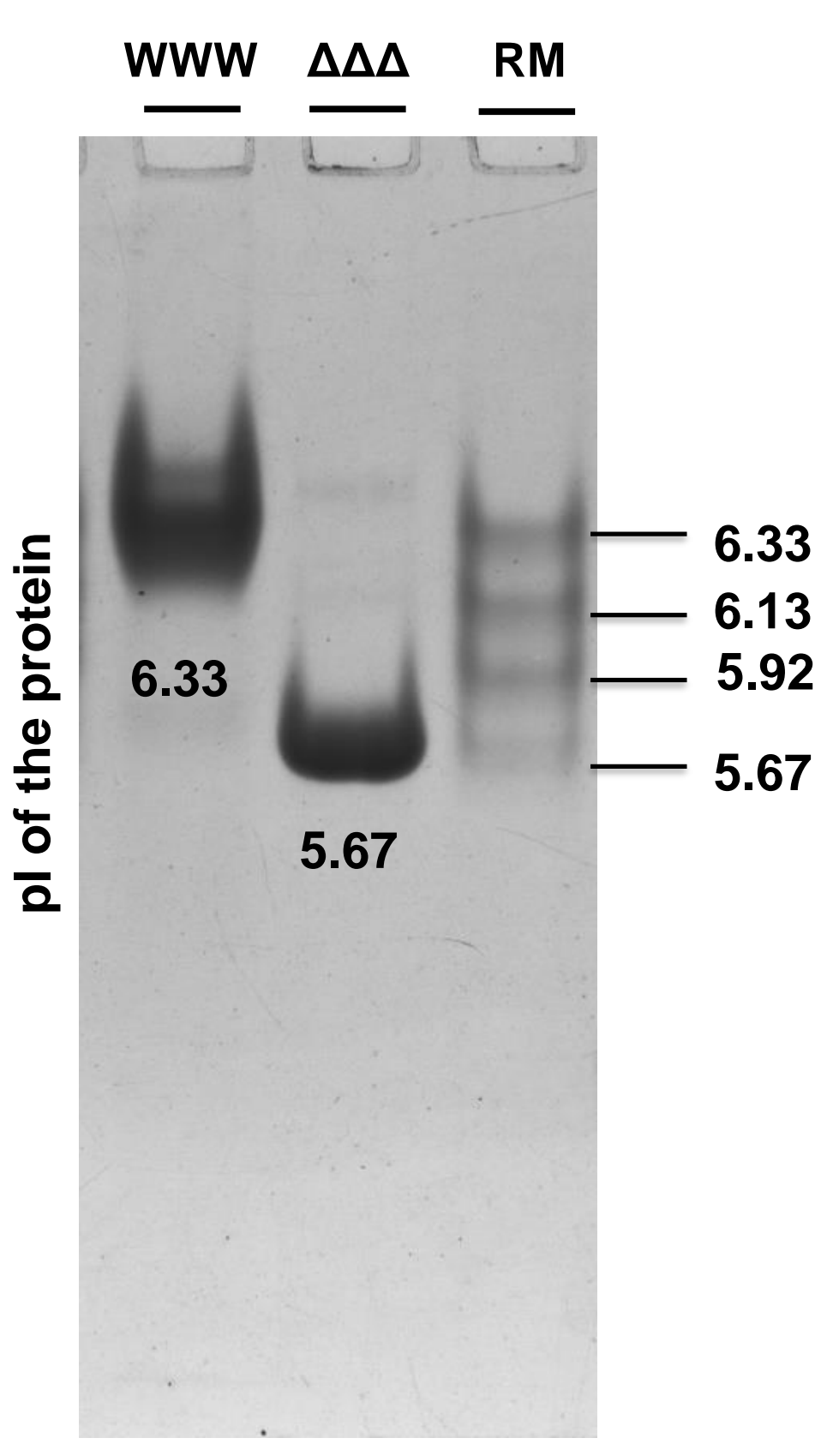

C

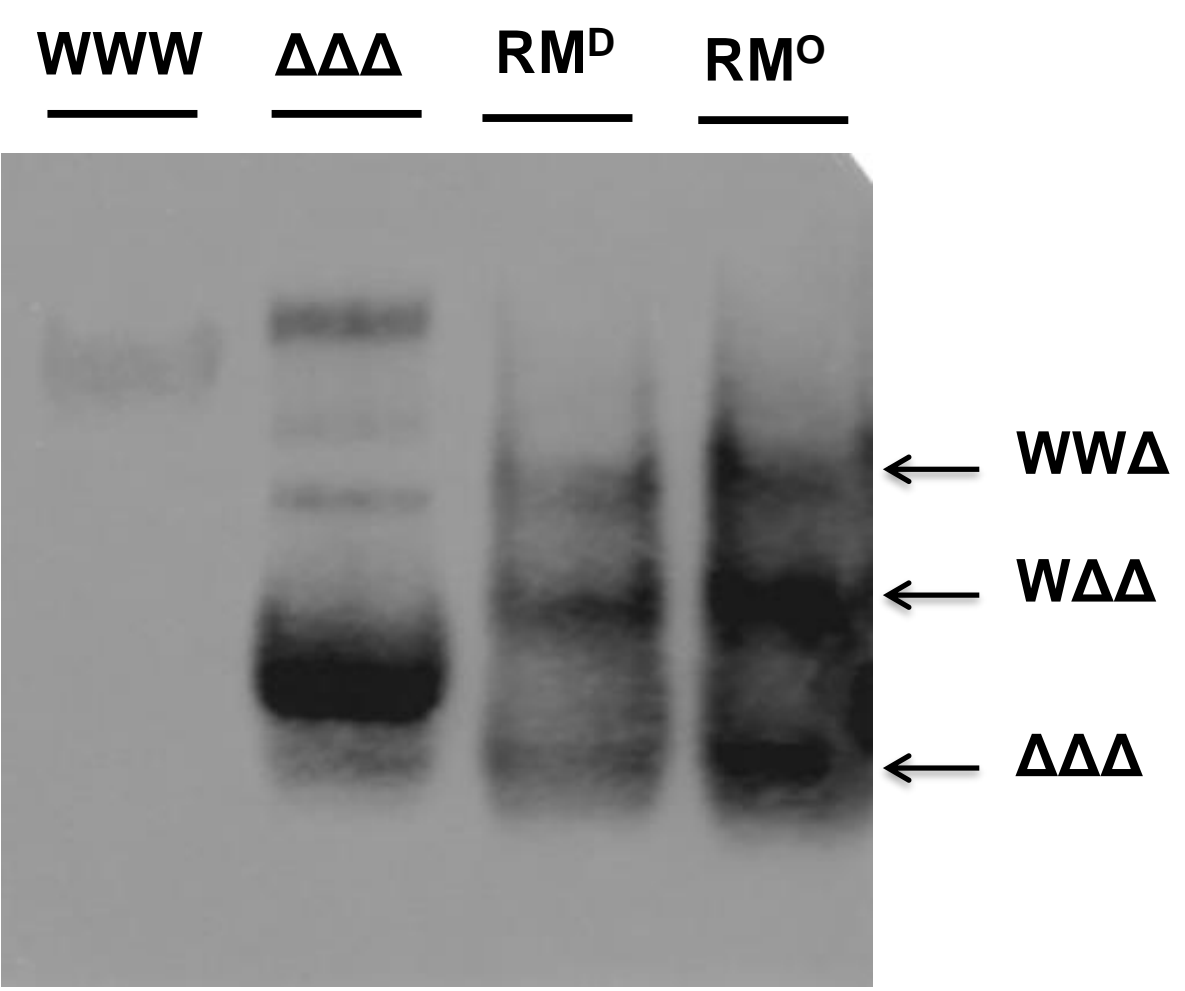

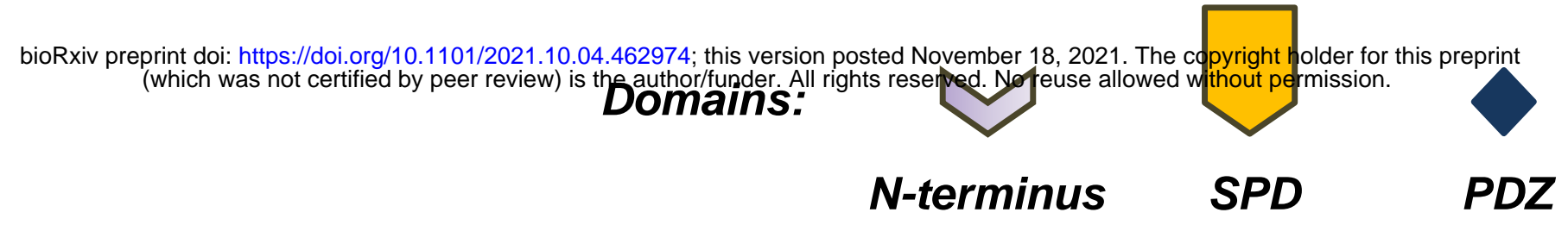

D

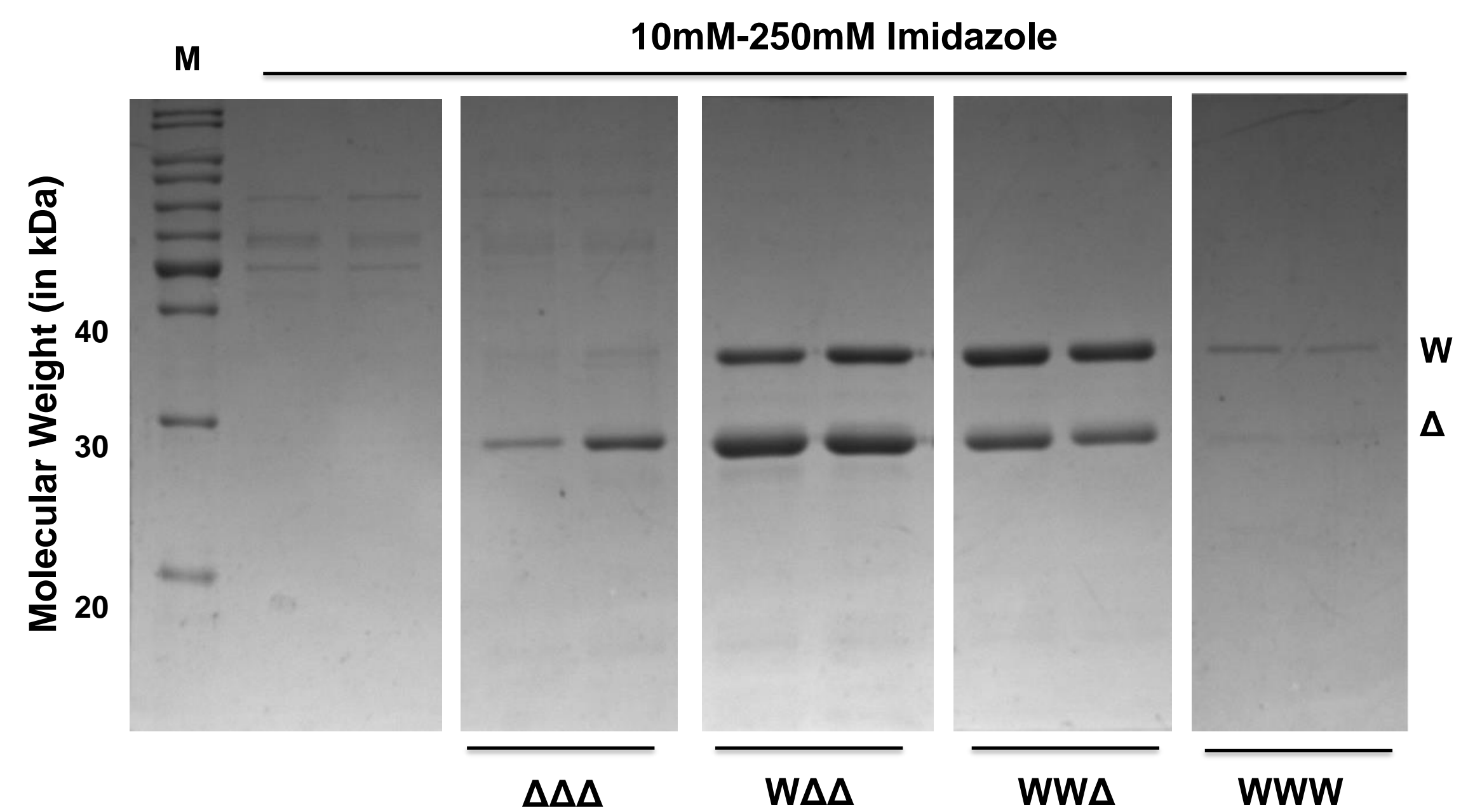

E

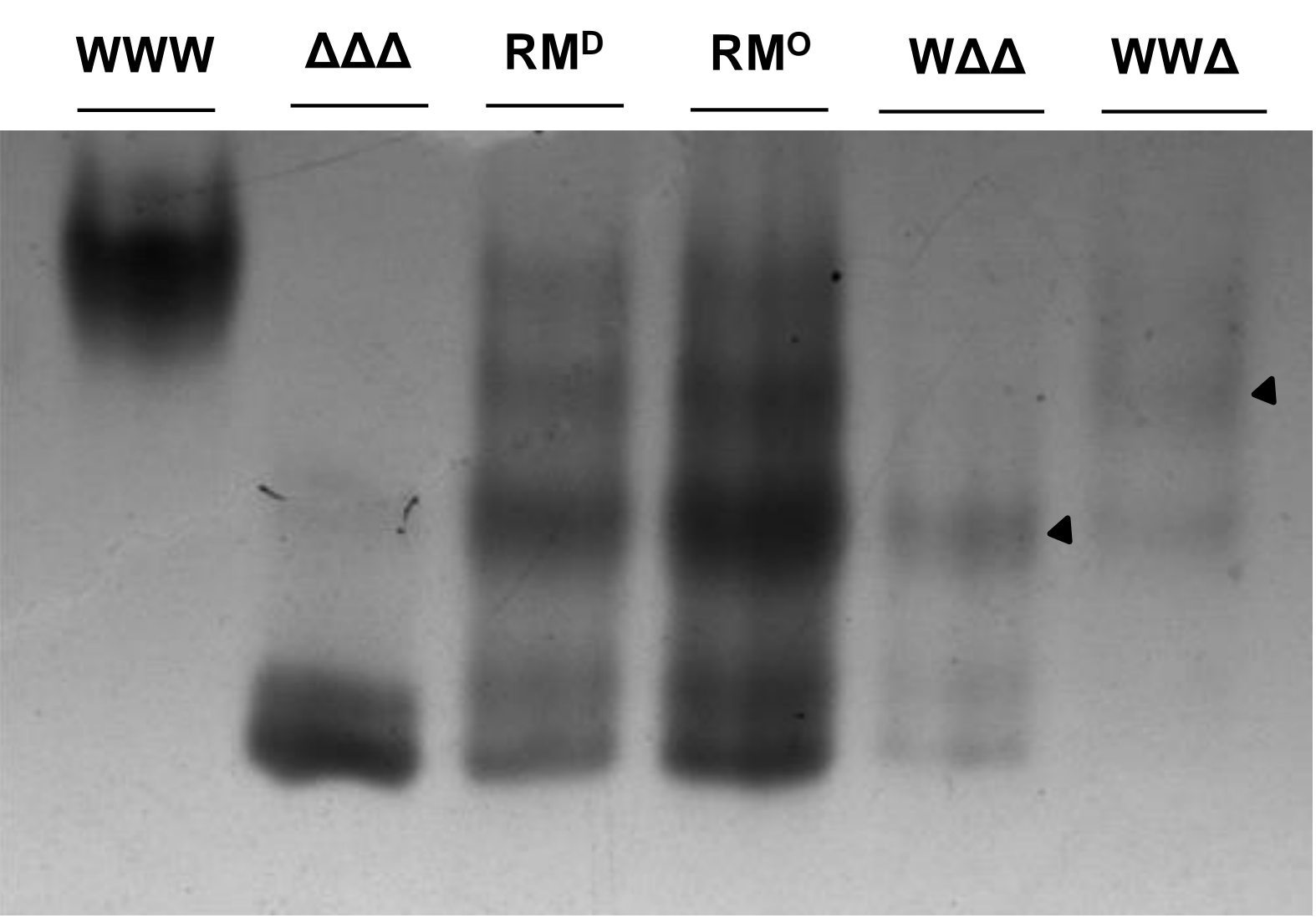


A
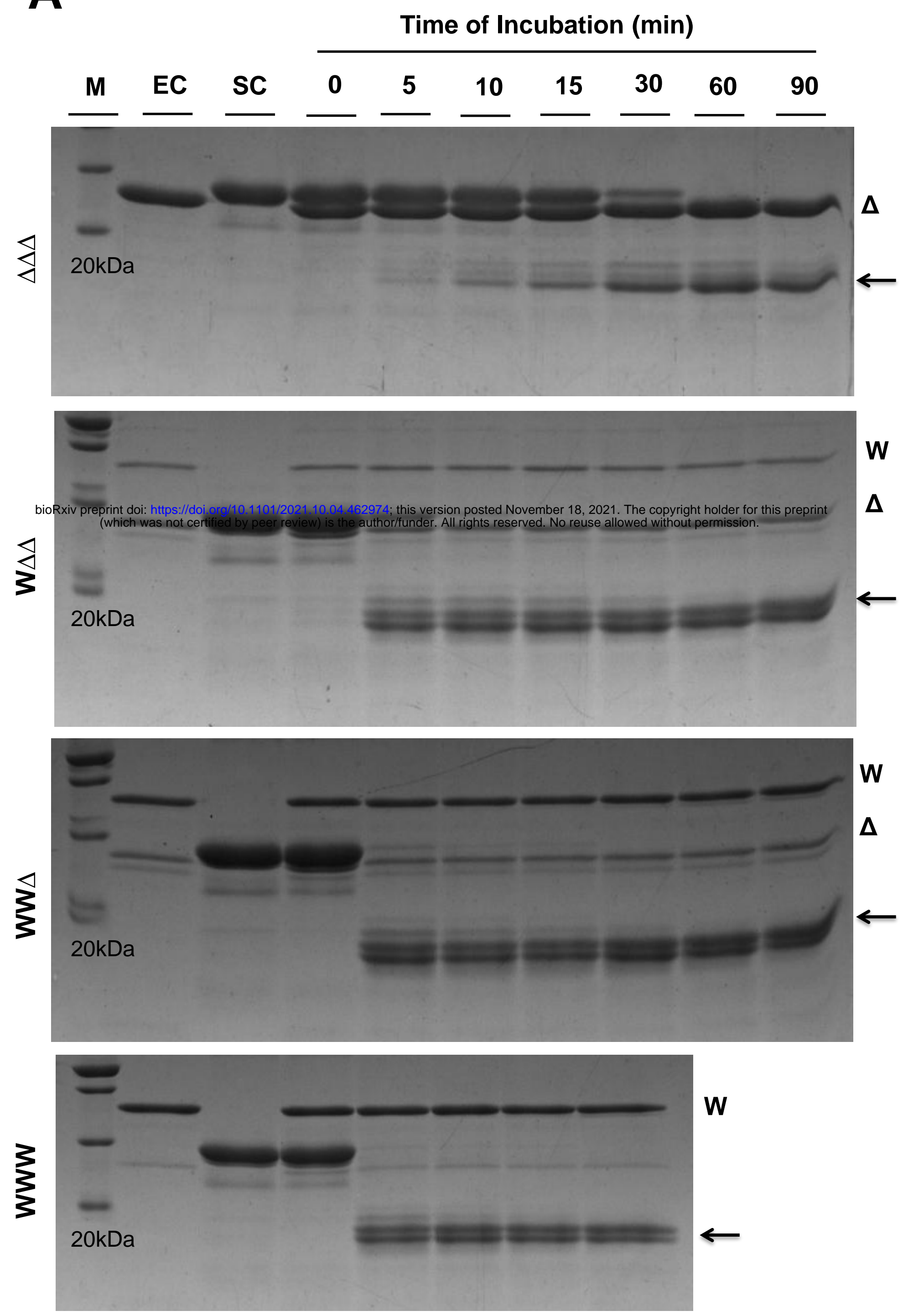

B

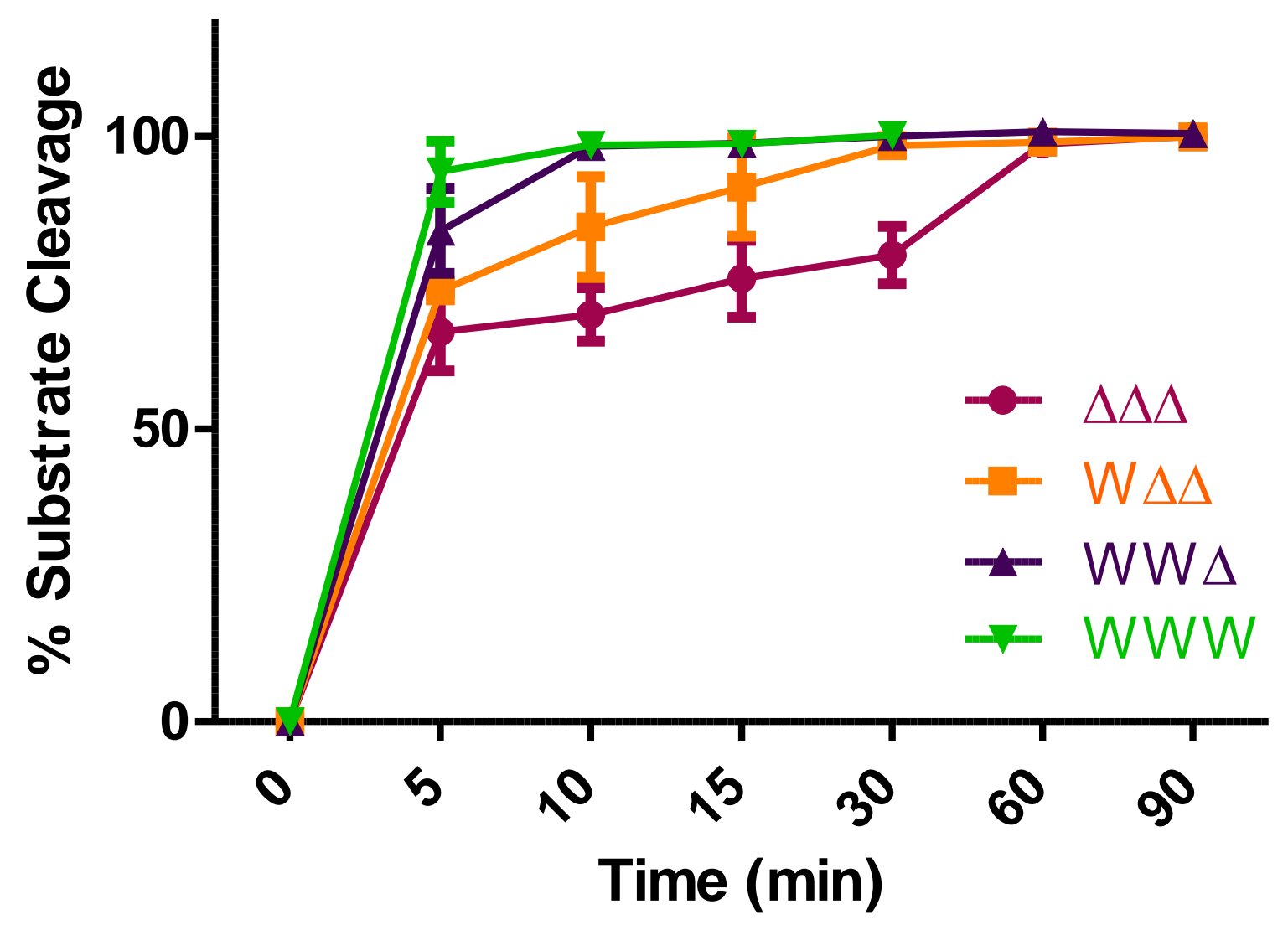

C

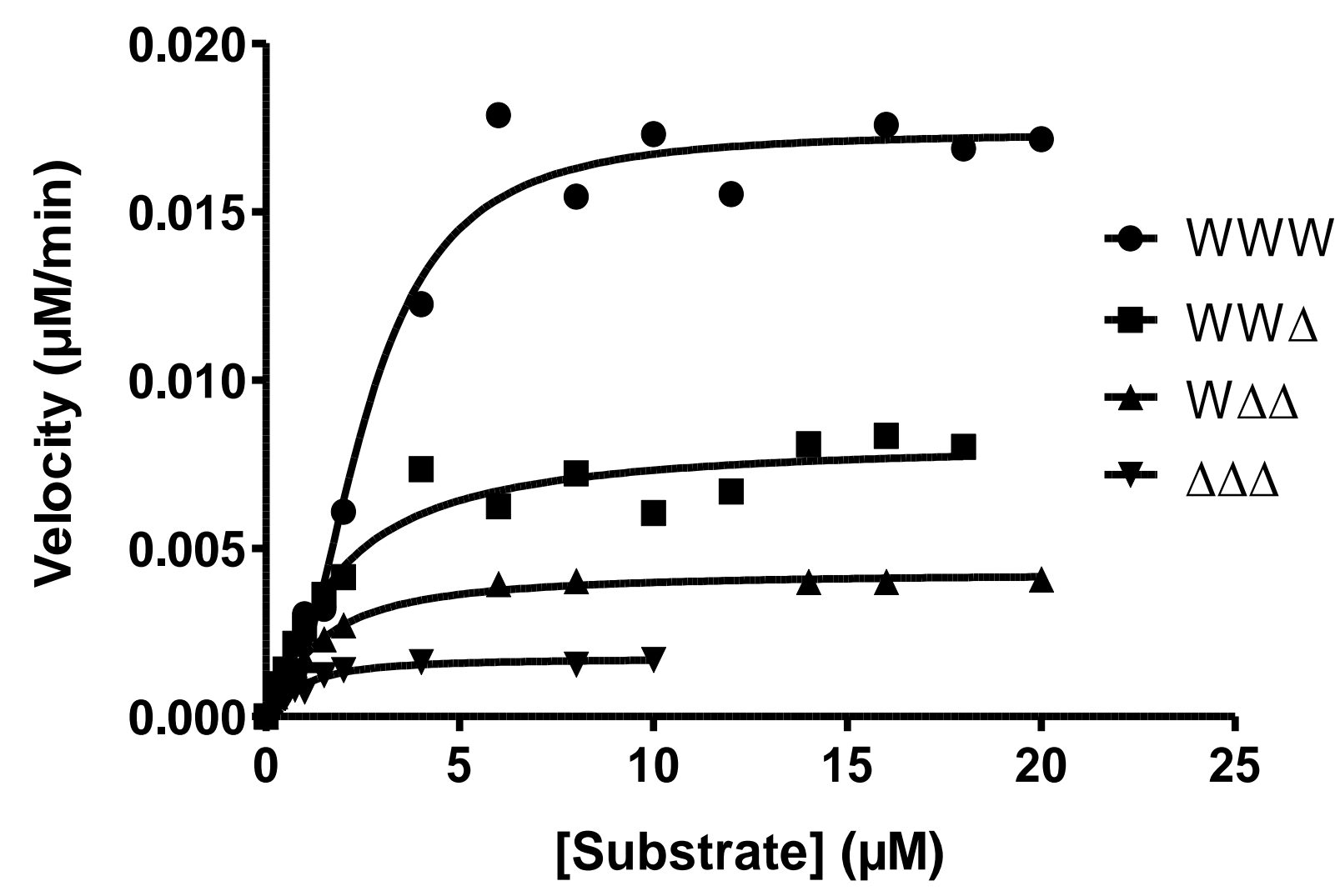


A

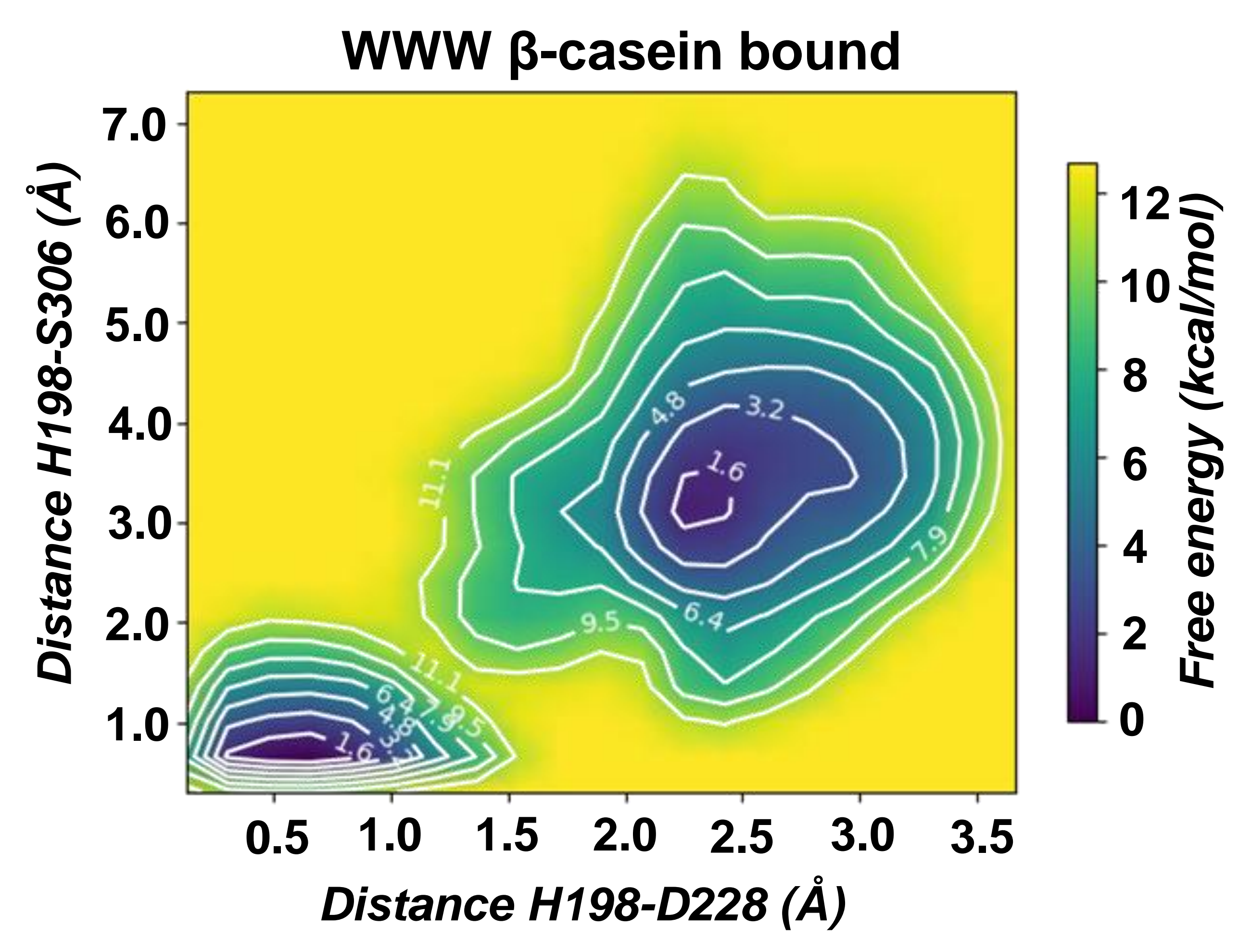

C

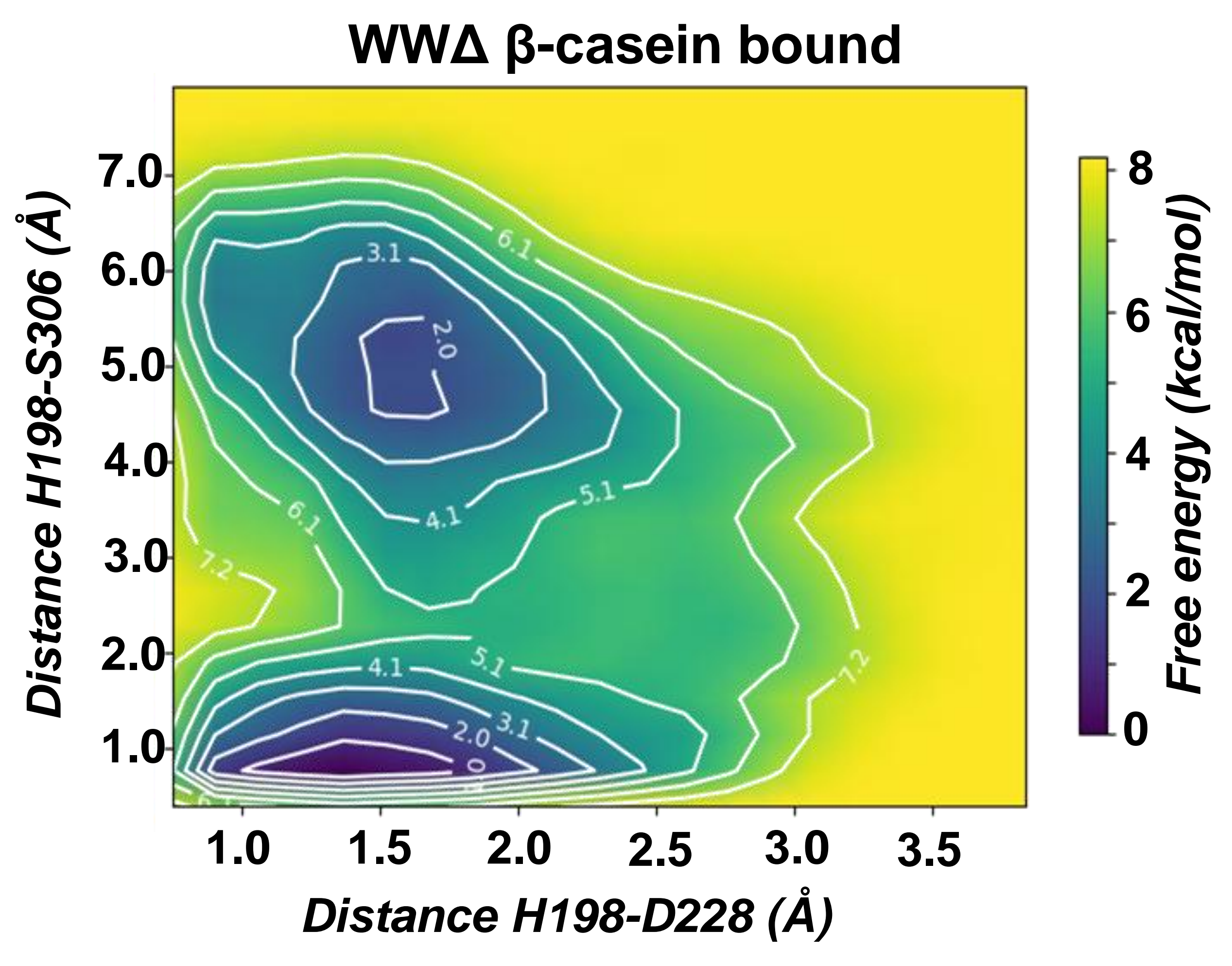

E

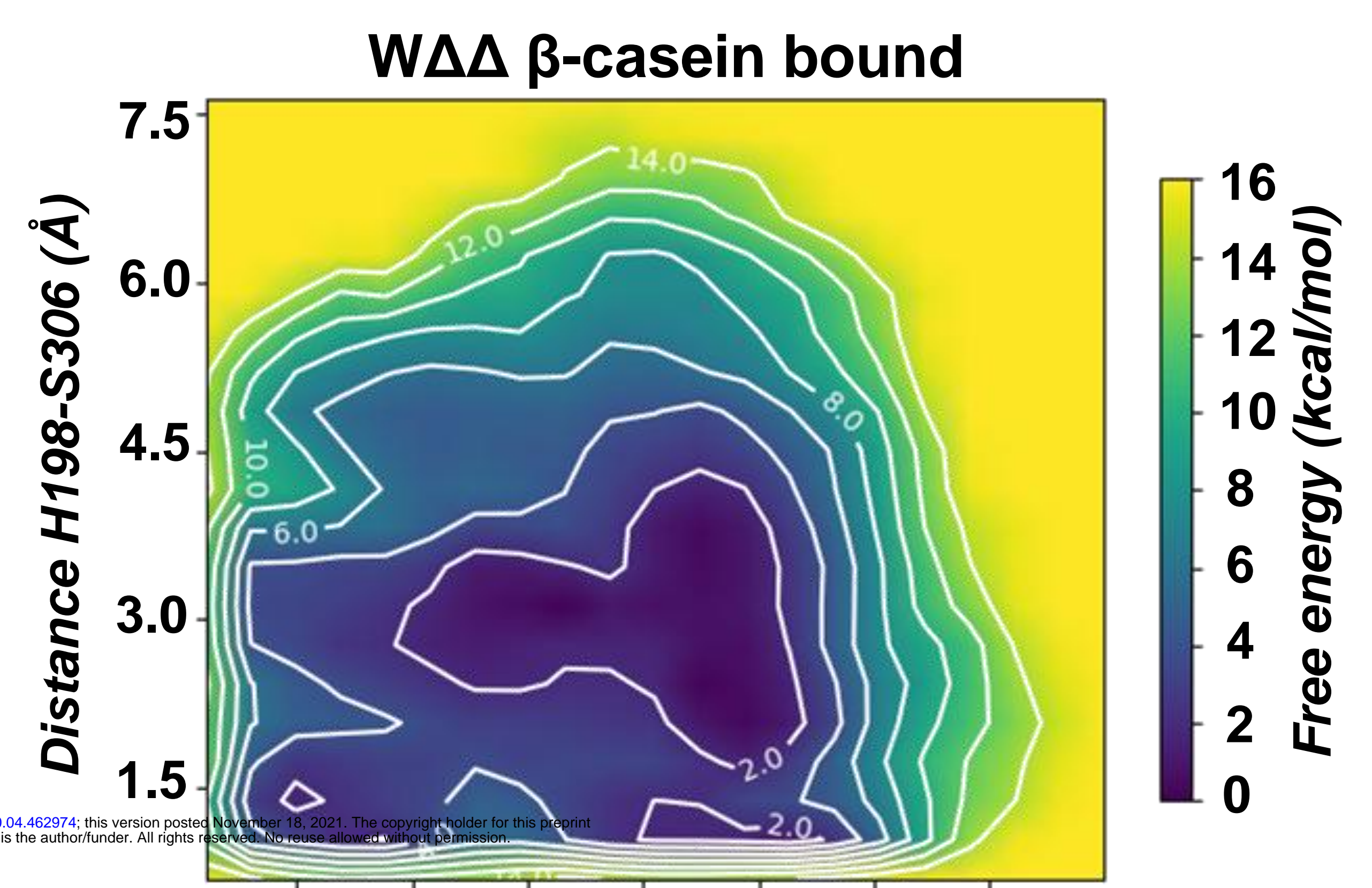

$\begin{array}{lllllll}0.5 & 1.0 & 1.5 & 2.0 & 2.5 & 3.0 & 3.5\end{array}$

Distance H198-D228 (Å)

G

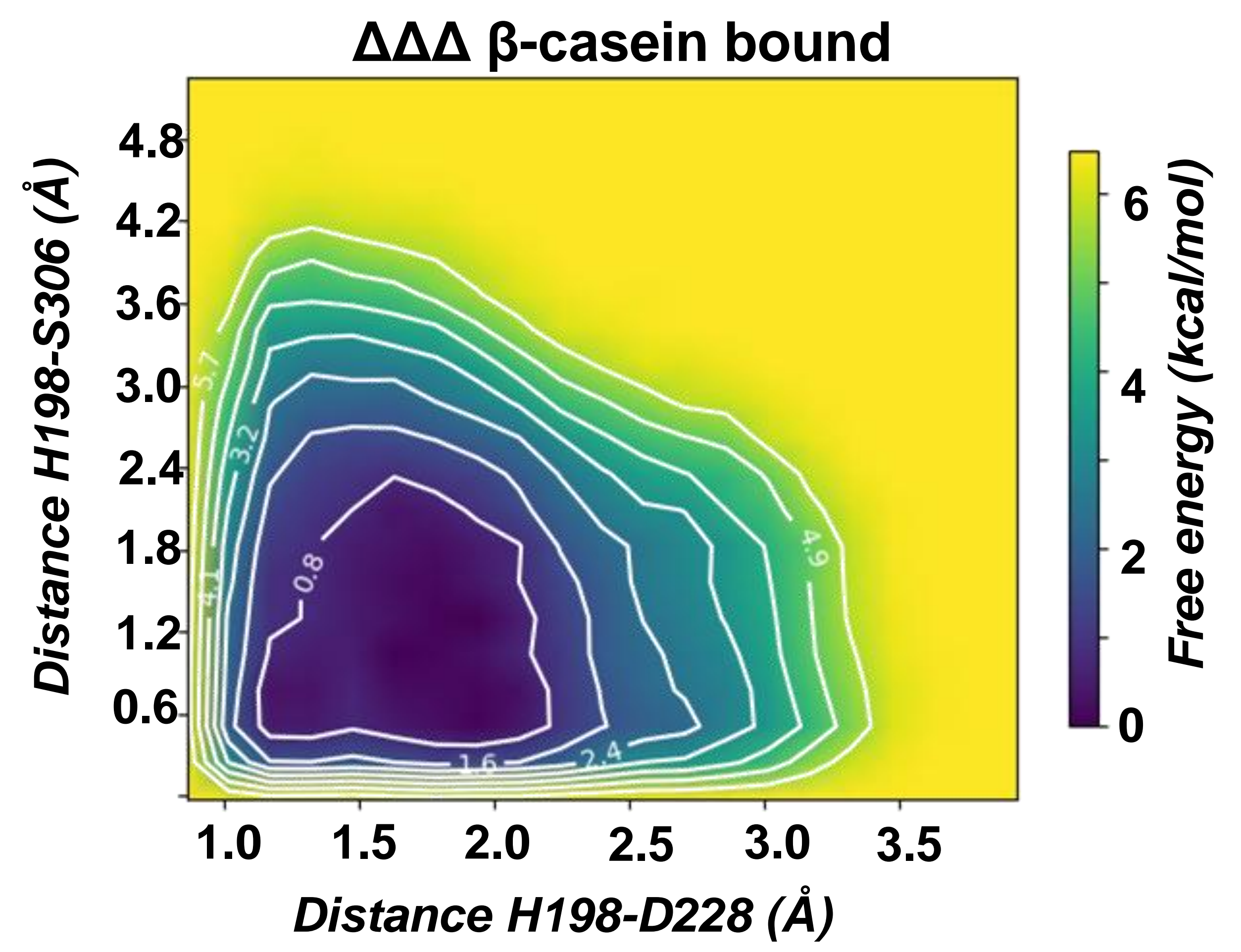

B

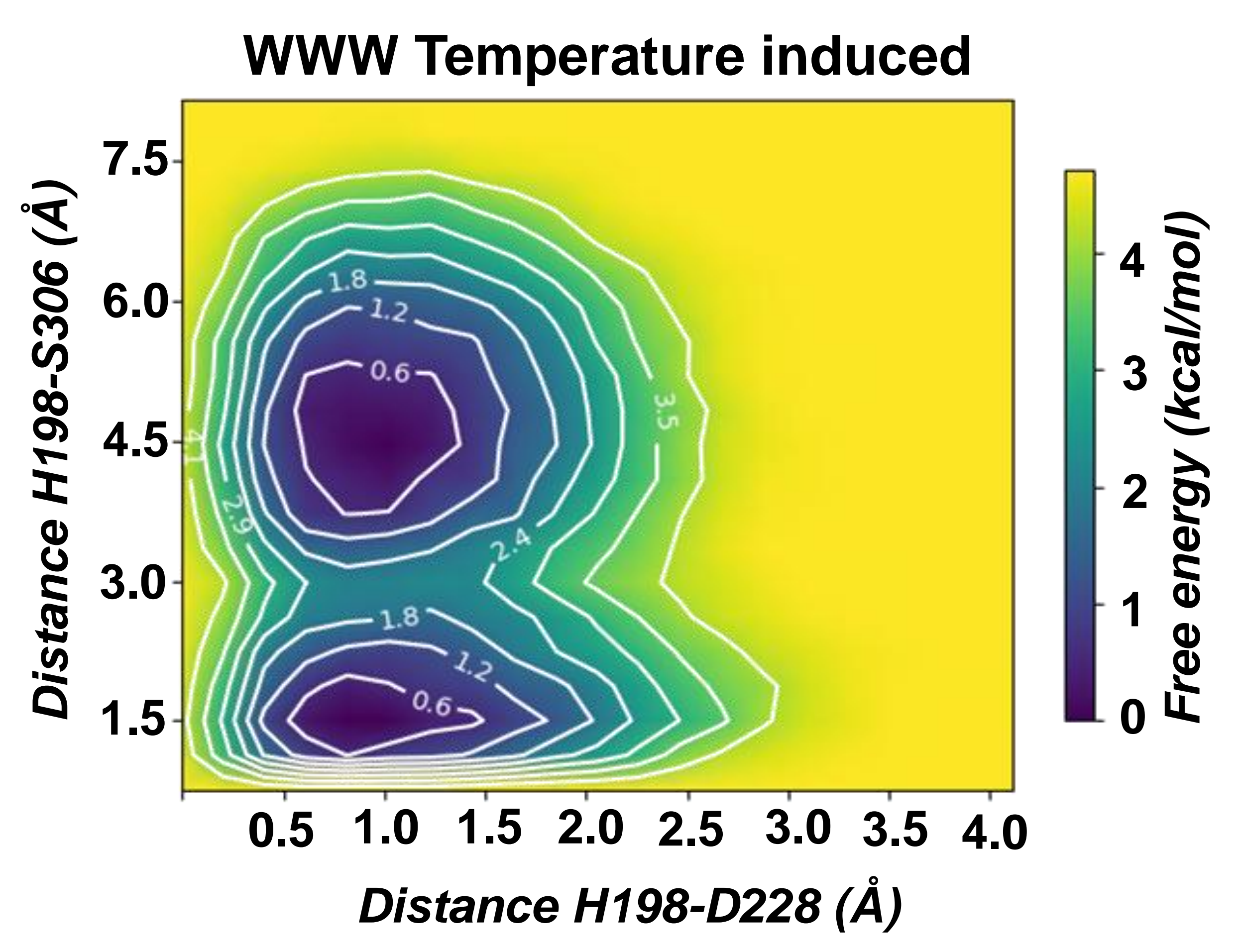

D

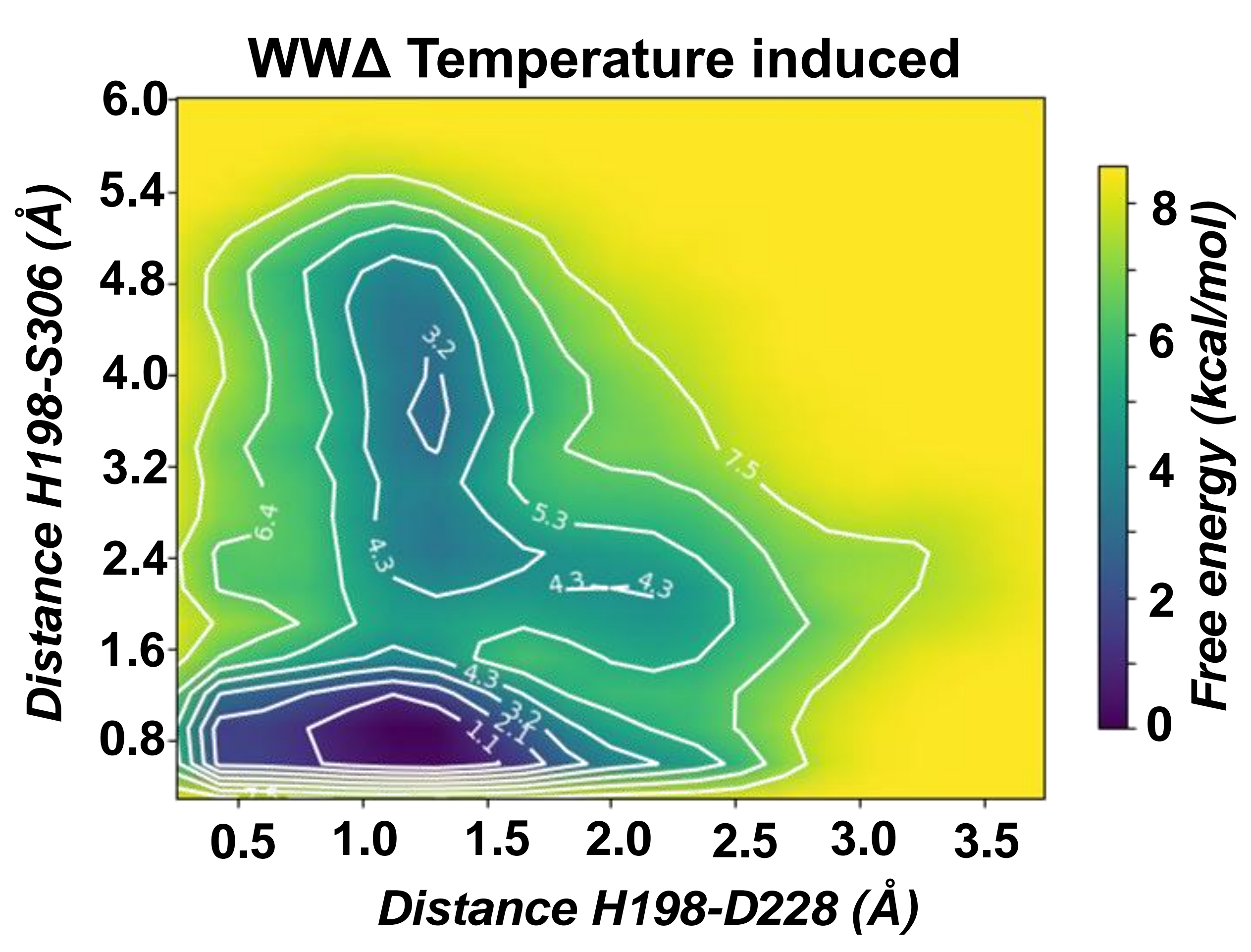

$\mathbf{F}$

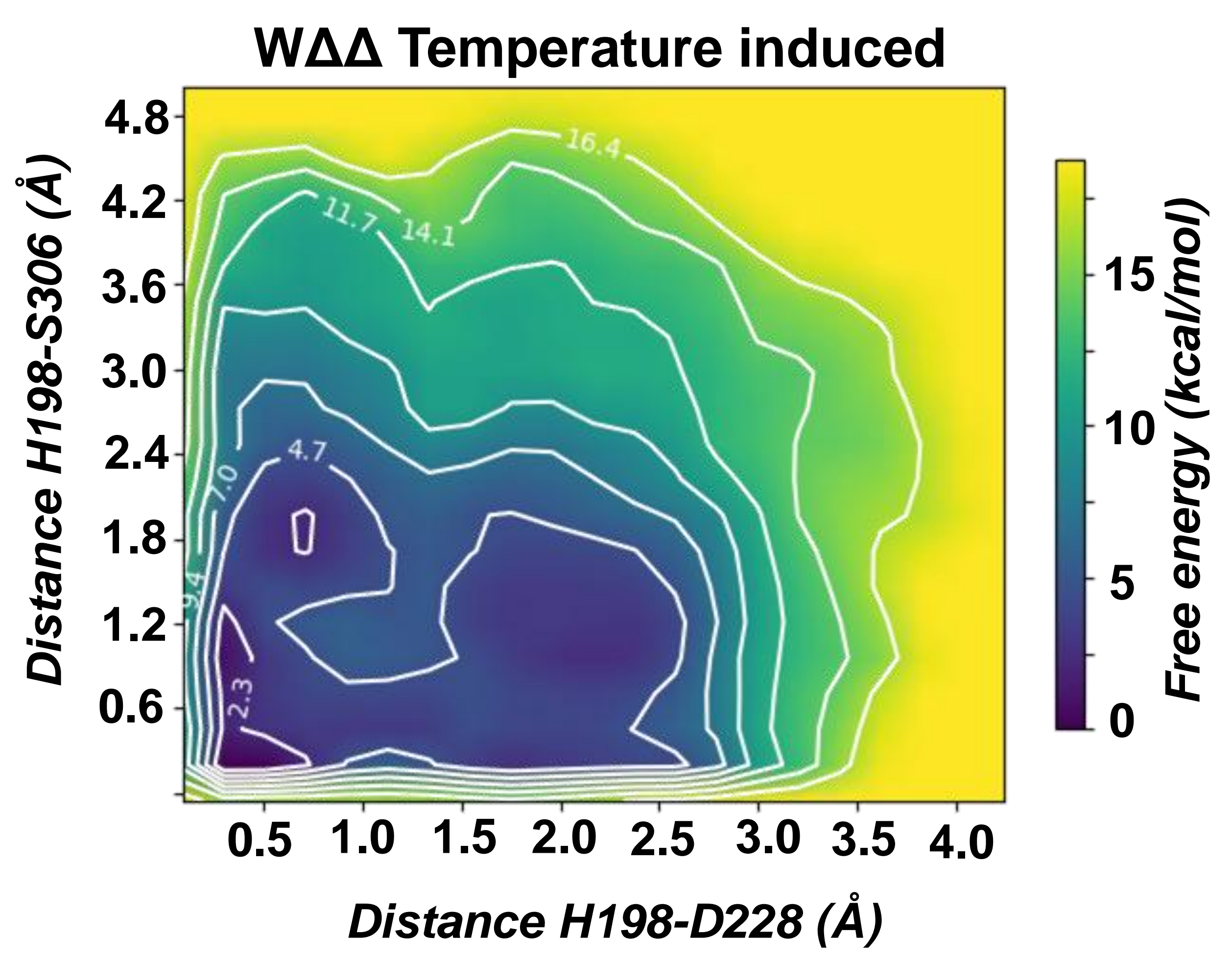

H

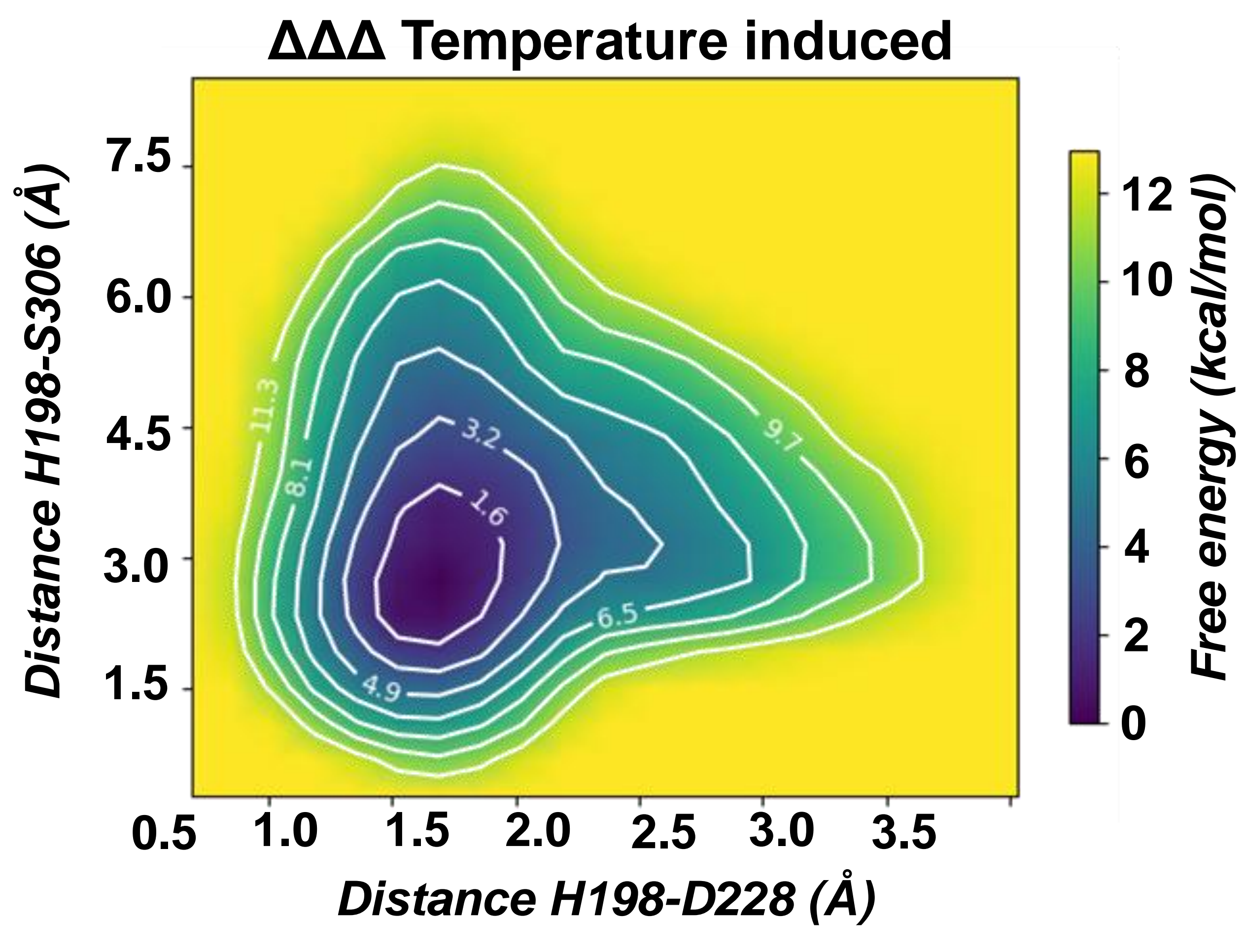


A

Substrate-induced activation

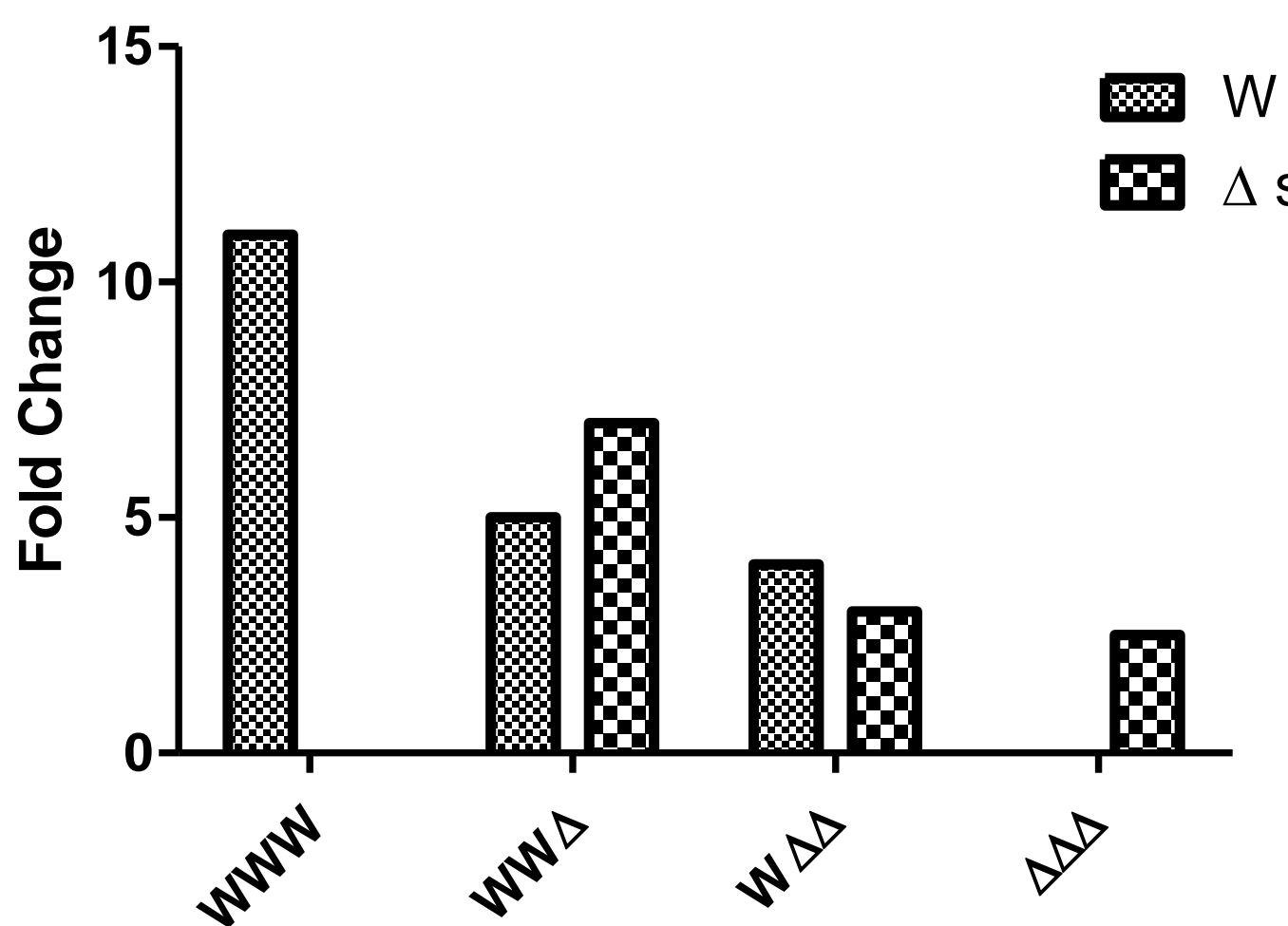

B

Temperature-induced activation

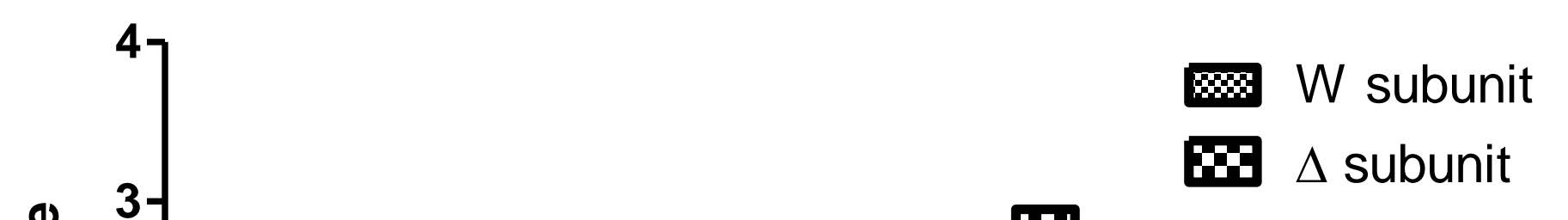

bioRxiv preprint doi: https:///doi.org/10.1101/2021.10.04.462974; this version posted November 18, 2021. The copyright holder for this preprint
(which was not certified by peer review) is the authorffunder. All rights reserved. No reuse allowed without permission.

C

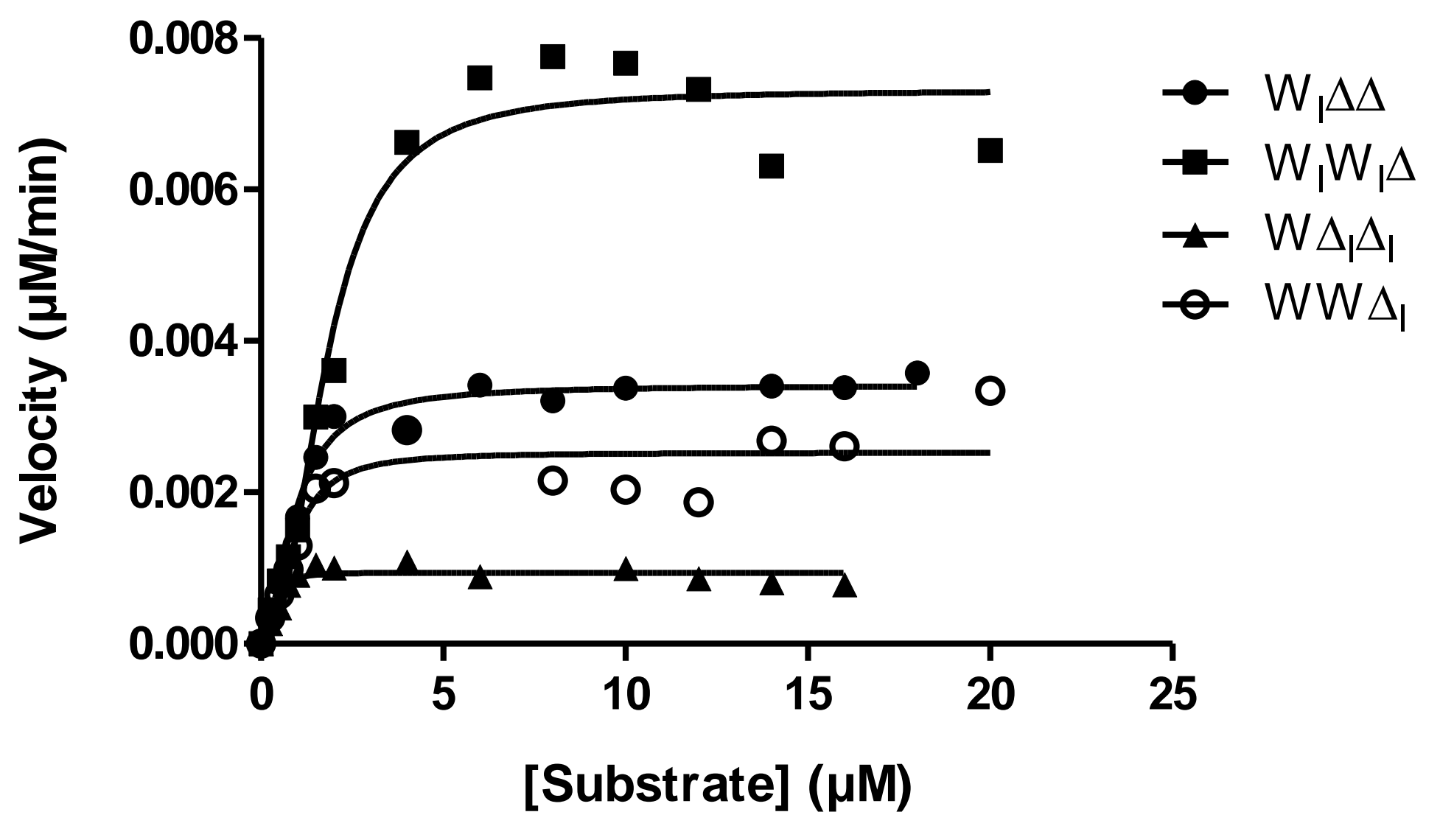

D

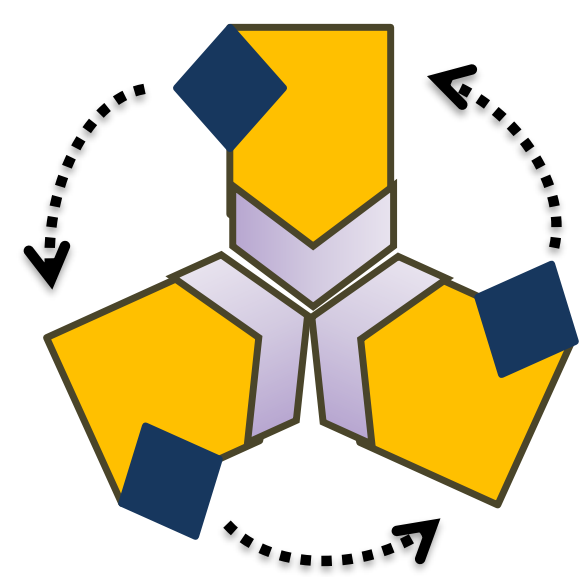

WWW

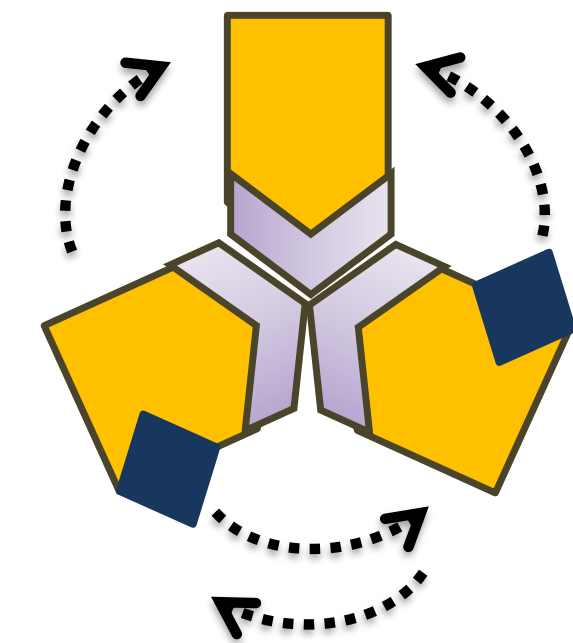

$\mathbf{W W} \Delta$

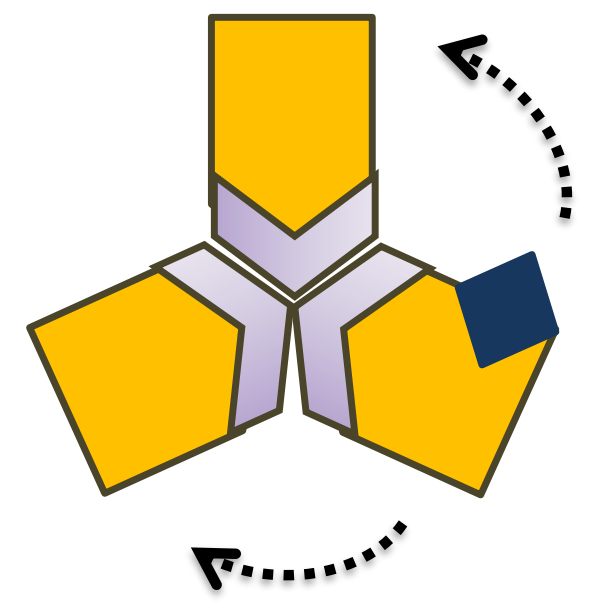

$\mathbf{W} \Delta \Delta$

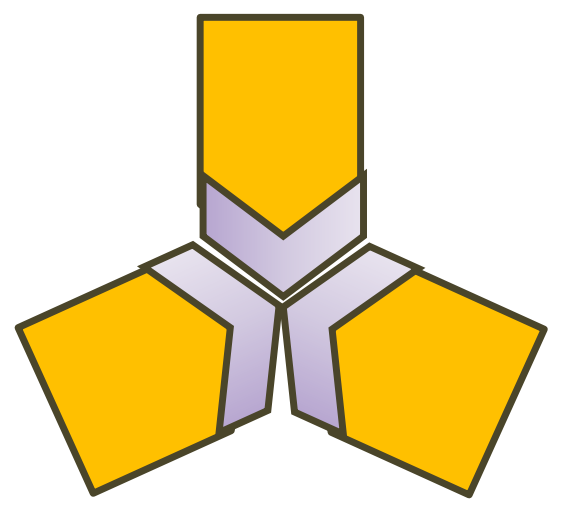

$\Delta \Delta \Delta$

trans-mediated allosteric communication 
A

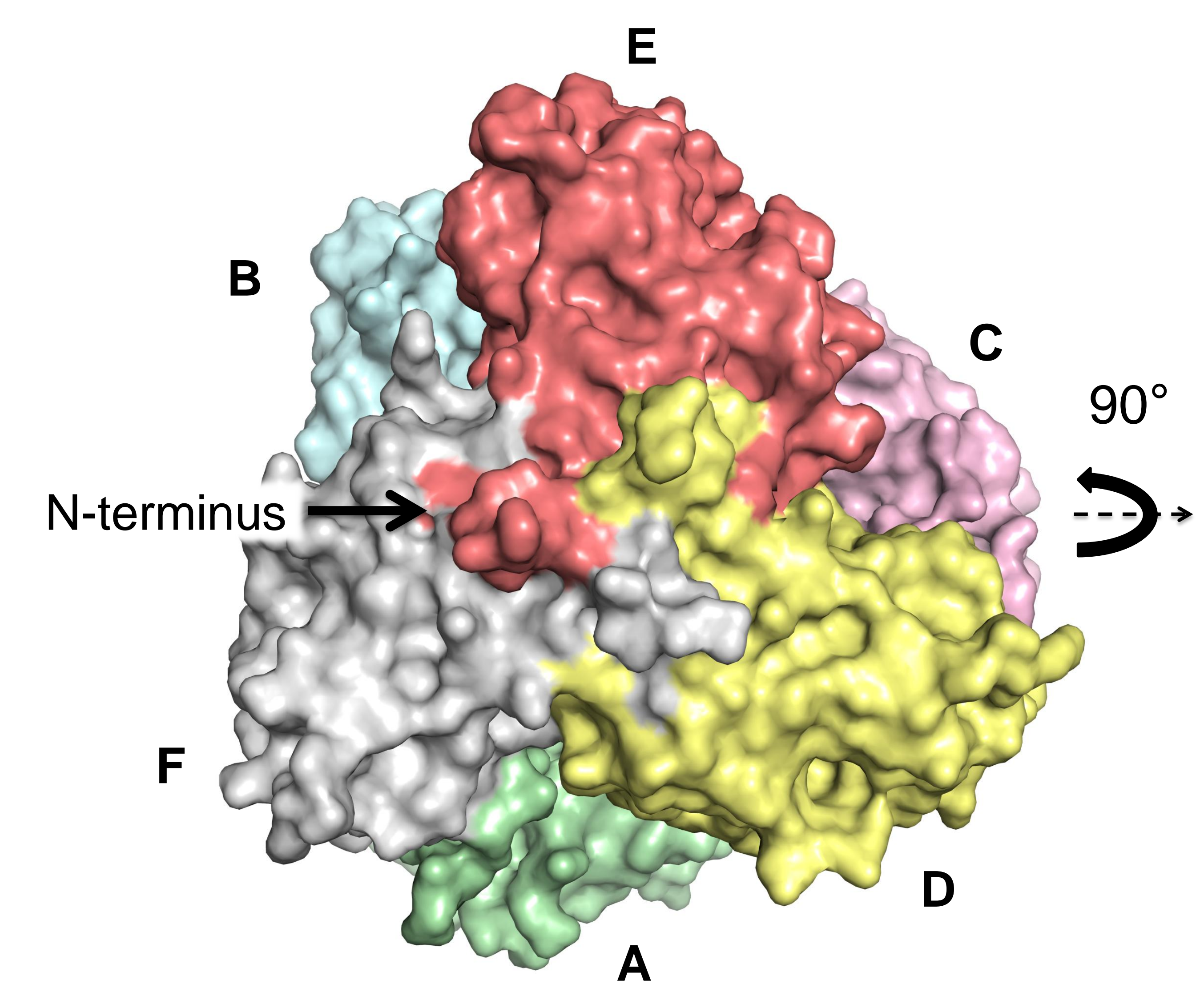

C

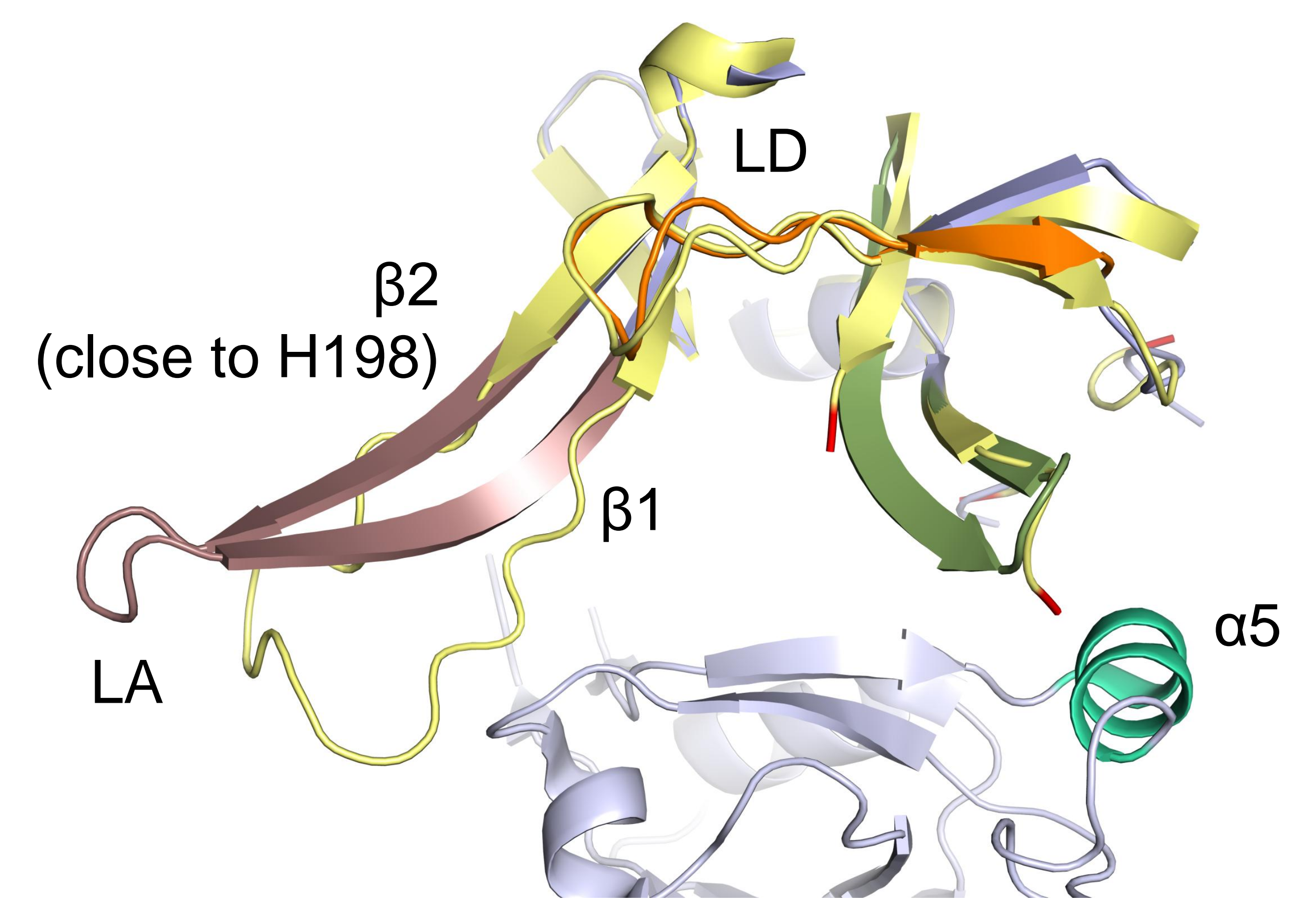

D

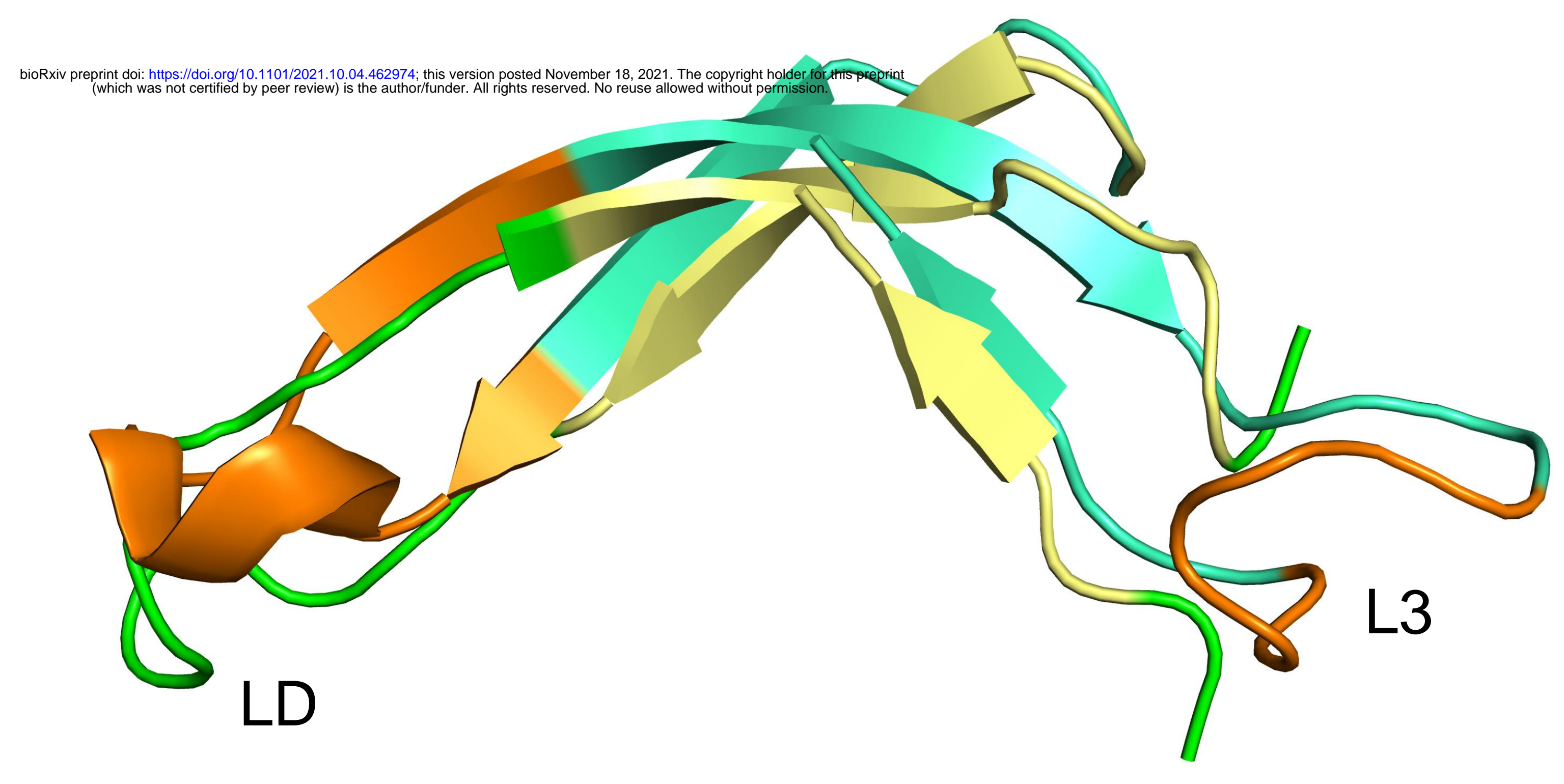

Trimer 2: Chains DEF

B

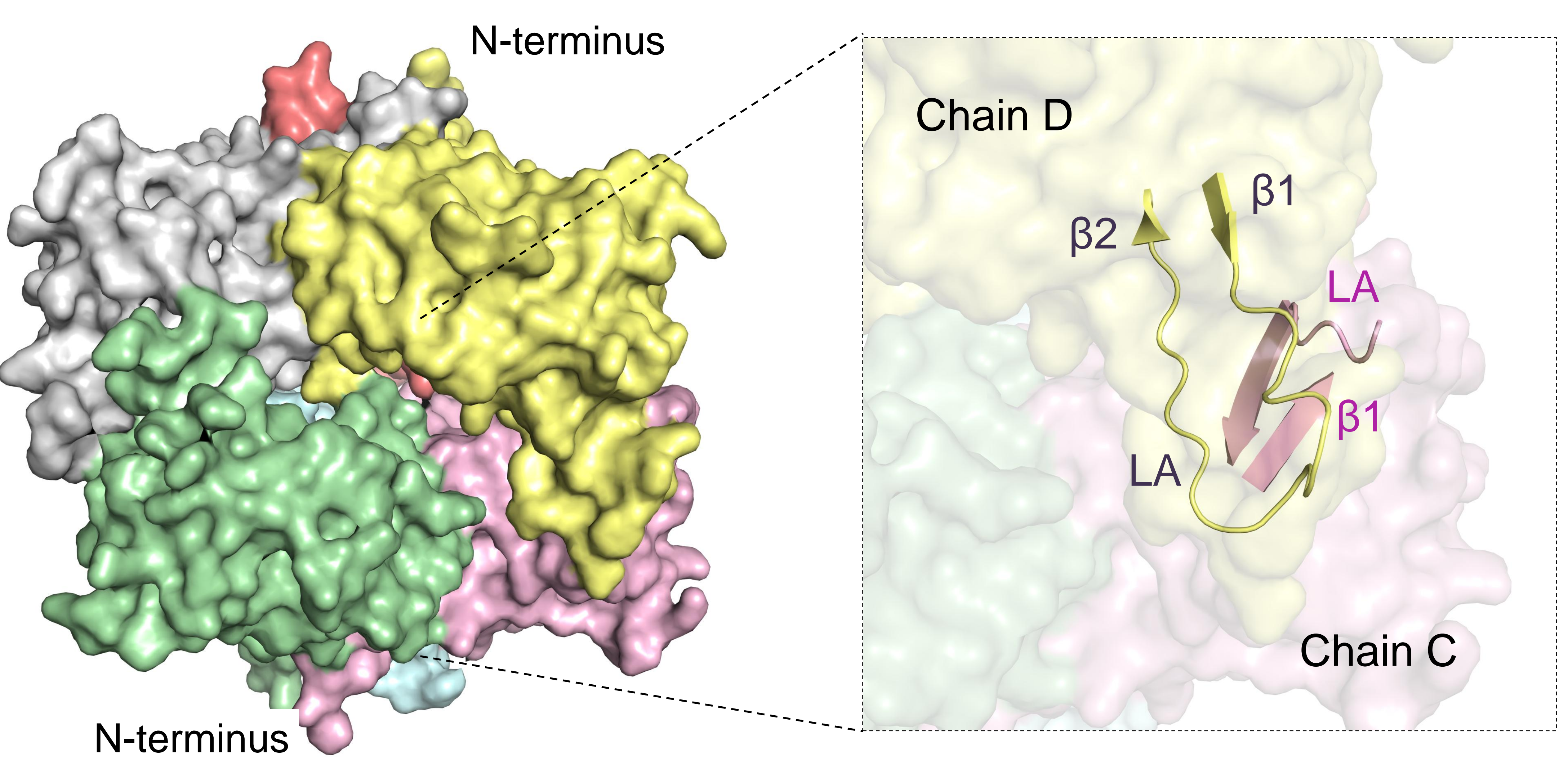

Trimer 1: Chains ABC
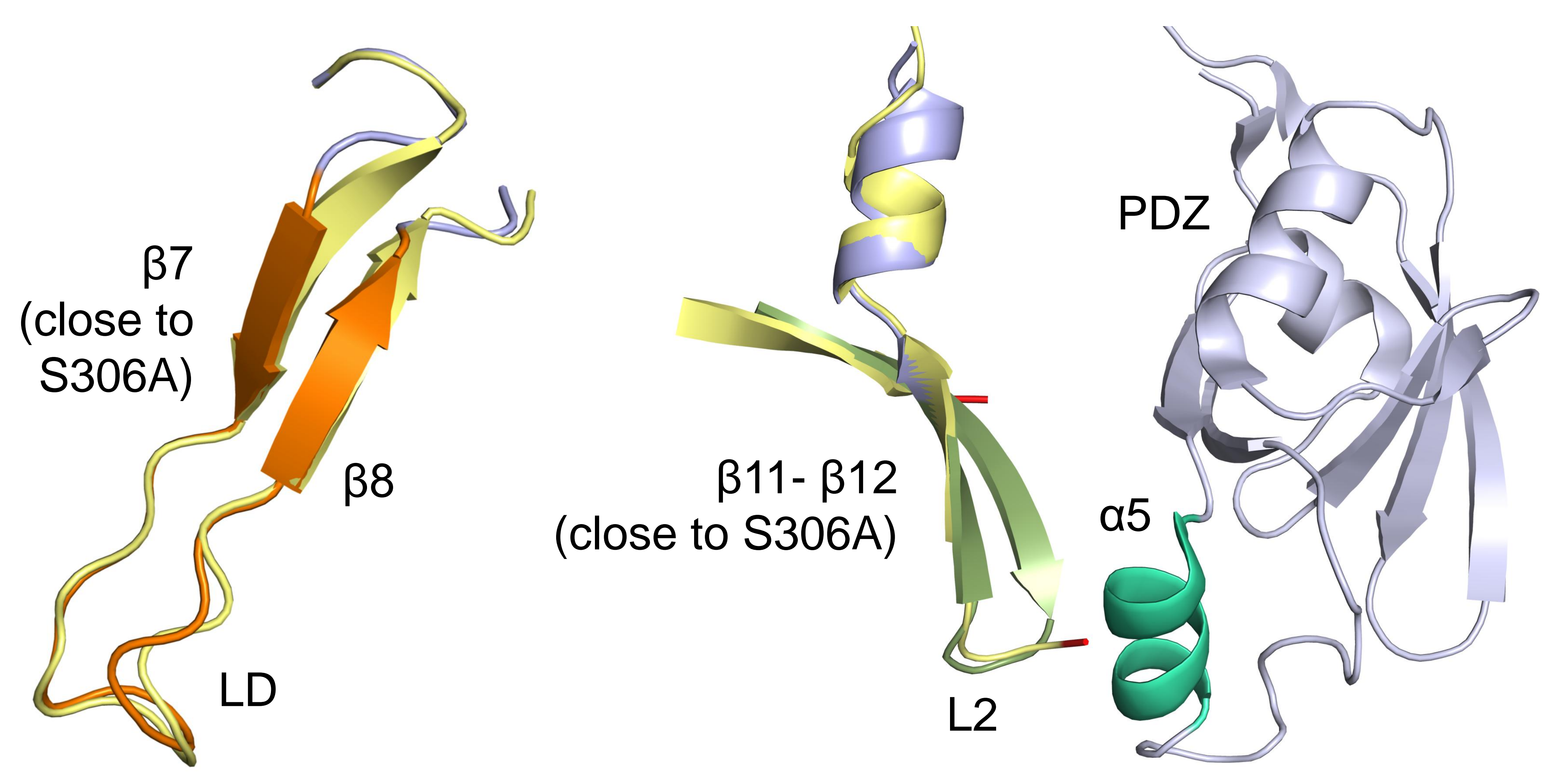

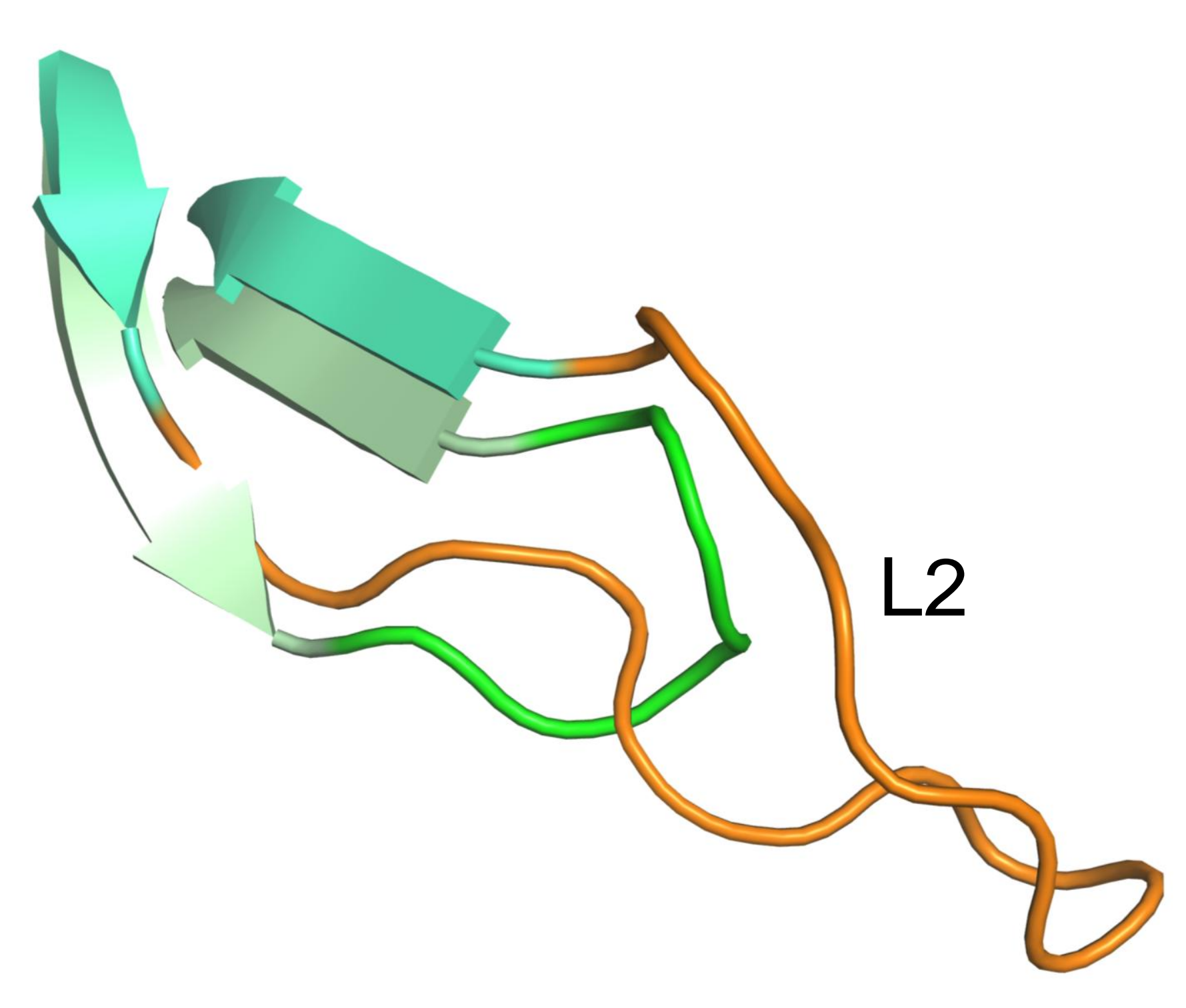


Initial binding displaces one PDZ and induces conformational changes in adjacent subunits (trans)
Increased N-terminal

region dynamics
(Ia)

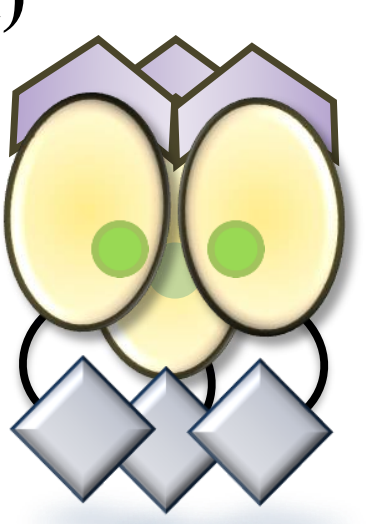

Inactive Basal state

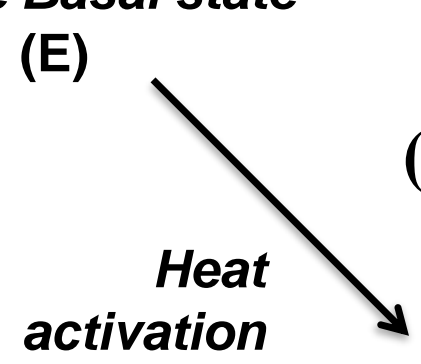

(II) ligand

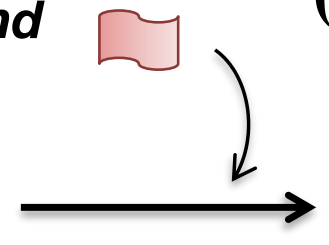

Substrate induced activation
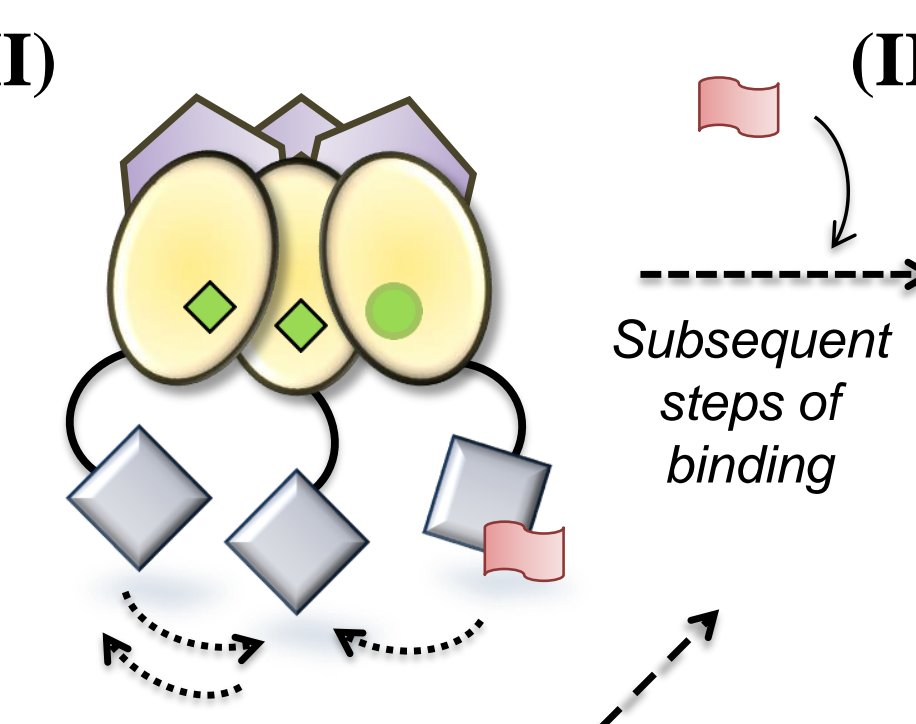

Subsequent steps of binding
(Ib)

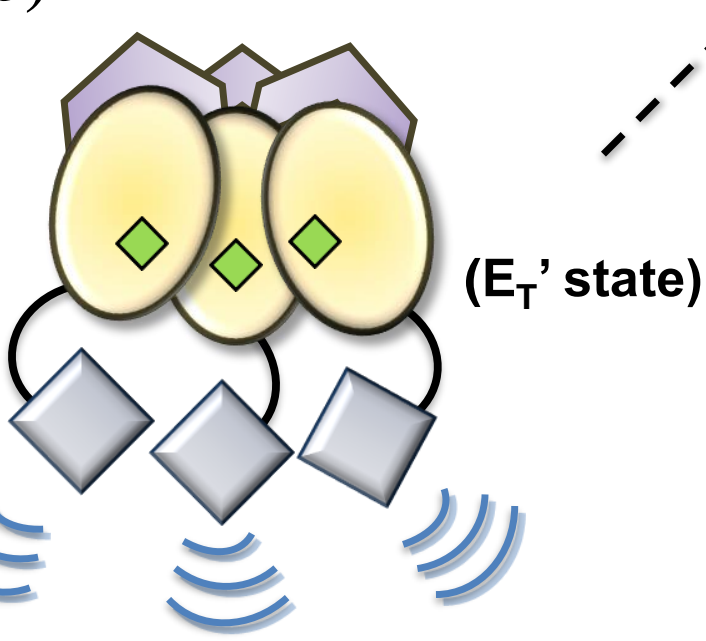

Increased thermal motion of the PDZ domains exposes the substrate binding pocket and induces active site rearrangement

\section{(III)}

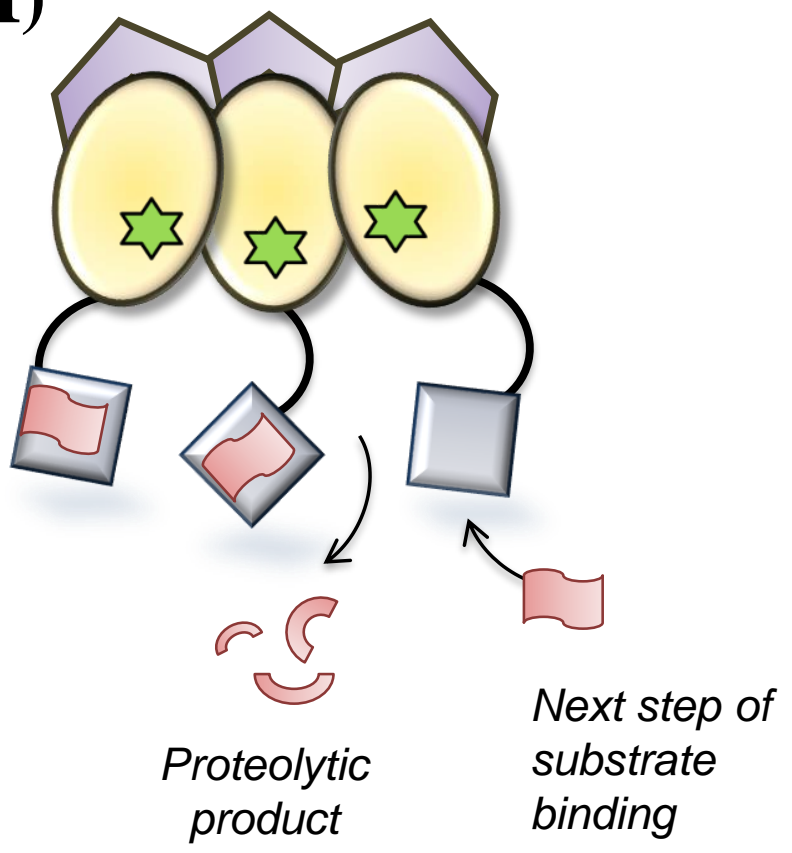

Catalytically active state

$\left(E^{*}\right)$
KEY:

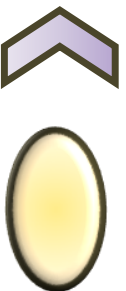
$N$-terminus
$\bigotimes P D Z$
Serine Protease
Domain
Flexible
Linker
Catalytic triad:
Inactive $\diamond$ Intermediate $\hat{\xi}$ Active 


\section{Supplemental Information}

Table S1: Top 5 docking scores generated for $\beta$-casein-bound $W W W, W W \Delta, W \Delta \Delta$ and $\Delta \mathbf{\Delta} \boldsymbol{\Delta}$

\begin{tabular}{|c|c|c|}
\hline HtrA2 Variant & Rank & $\begin{array}{c}\beta \text {-casein-bound } \\
\text { (kcal/mol) }\end{array}$ \\
\hline \multirow[t]{5}{*}{ WWW } & Rank 1 & -6.9 \\
\hline & Rank 2 & -6.5 \\
\hline & Rank 3 & -5.9 \\
\hline & Rank 4 & -5.5 \\
\hline & Rank 5 & -5.4 \\
\hline \multirow[t]{5}{*}{ WW } & Rank 1 & -7.3 \\
\hline & Rank 2 & -6.8 \\
\hline & Rank 3 & -6.3 \\
\hline & Rank 4 & -5.6 \\
\hline & Rank 5 & -5.6 \\
\hline \multirow[t]{5}{*}{$\mathrm{W} \Delta \Delta$} & Rank 1 & -7.9 \\
\hline & Rank 2 & -7.3 \\
\hline & Rank 3 & -6.5 \\
\hline & Rank 4 & -6.4 \\
\hline & Rank 5 & -5.5 \\
\hline \multirow[t]{5}{*}{$\Delta \Delta \Delta$} & Rank 1 & -8.7 \\
\hline & Rank 2 & -7.4 \\
\hline & Rank 3 & -7.2 \\
\hline & Rank 4 & -6.6 \\
\hline & Rank 5 & -6.5 \\
\hline
\end{tabular}


Table S2: Average distance (in $\AA$ ) analysis among the catalytic triad residues for HtrA2 variants. The distances were calculated over a $1000 \mathrm{~ns}$ time-scale for $\beta$-casein-bound and temperature induced WWW, WW $\Delta, \mathrm{W} \Delta \Delta$ and $\Delta \Delta \Delta$.

\begin{tabular}{|c|c|c|c|c|c|c|c|c|}
\hline \multirow{3}{*}{ Residue } & \multicolumn{8}{|c|}{$\beta$-casein-bound (in $\AA$ ) } \\
\hline & \multicolumn{2}{|c|}{ WWW } & \multicolumn{2}{|c|}{ WW } & \multicolumn{2}{|c|}{$\mathbf{W} \Delta \mathbf{\Delta}$} & \multicolumn{2}{|c|}{$\Delta \Delta \Delta$} \\
\hline & Unbound & Bound & Unbound & Bound & Unbound & Bound & Unbound & Bound \\
\hline $\begin{array}{c}\text { ChainA } \\
\text { H198 to } \\
\text { D228 }\end{array}$ & $2.9 \pm 0.2$ & $2.6 \pm 0.1$ & $2.9 \pm 0.3$ & $2.8 \pm 0.1$ & $3.0 \pm 0.2$ & $2.9 \pm 0.3$ & $2.9 \pm 0.2$ & $3.2 \pm 0.2$ \\
\hline $\begin{array}{c}\text { ChainA } \\
\text { H198 to } \\
\text { S306 }\end{array}$ & $4.1 \pm 0.2$ & $4.9 \pm 0.1$ & $4.2 \pm 0.2$ & $4.5 \pm 0.2$ & $4.2 \pm 0.2$ & $4.2 \pm 0.1$ & $4.1 \pm 0.2$ & $3.7 \pm 0.2$ \\
\hline $\begin{array}{c}\text { ChainB } \\
\text { H198 to } \\
\text { D228 }\end{array}$ & $2.9 \pm 0.2$ & $2.7 \pm 0.2$ & $2.9 \pm 0.3$ & $2.7 \pm 0.1$ & $3.0 \pm 0.3$ & $3.0 \pm 0.1$ & $2.9 \pm 0.2$ & $3.1 \pm 0.2$ \\
\hline $\begin{array}{c}\text { ChainB } \\
\text { H198 to } \\
\text { S306 }\end{array}$ & $4.1 \pm 0.2$ & $5.3 \pm 0.2$ & $4.2 \pm 0.2$ & $4.4 \pm 0.1$ & $4.2 \pm 0.2$ & $4.3 \pm 0.2$ & $4.1 \pm 0.2$ & $3.6 \pm 0.1$ \\
\hline $\begin{array}{c}\text { ChainC } \\
\text { H198 to } \\
\text { D228 }\end{array}$ & $2.9 \pm 0.2$ & $2.5 \pm 0.2$ & $2.9 \pm 0.3$ & $2.6 \pm 0.2$ & $3.0 \pm 0.3$ & $2.7 \pm 0.3$ & $2.9 \pm 0.2$ & $3.2 \pm 0.1$ \\
\hline $\begin{array}{c}\text { ChainC } \\
\text { H198 to } \\
\text { S306 }\end{array}$ & $4.1 \pm 0.2$ & $5.1 \pm 0.2$ & $4.2 \pm 0.2$ & $4.5 \pm 0.1$ & $4.2 \pm 0.2$ & $4.4 \pm 0.1$ & $4.1 \pm 0.2$ & $3.7 \pm 0.3$ \\
\hline \multirow{3}{*}{ Residue } & \multicolumn{8}{|c|}{ Temperature-induced activation (in $\mathbf{A}$ ) } \\
\hline & \multicolumn{2}{|c|}{ WWW } & \multicolumn{2}{|c|}{$\mathbf{W W \Delta}$} & \multicolumn{2}{|c|}{$\mathbf{W} \Delta \mathbf{\Delta}$} & \multicolumn{2}{|c|}{$\Delta \Delta \Delta$} \\
\hline & Uninduced & Induced & $\begin{array}{c}\text { Uninduce } \\
\text { d }\end{array}$ & Induced & $\begin{array}{c}\text { Uninduc } \\
\text { ed }\end{array}$ & Induced & $\begin{array}{c}\text { Uninduce } \\
\text { d }\end{array}$ & Induced \\
\hline $\begin{array}{c}\text { ChainA } \\
\text { H198 to } \\
\text { D228 }\end{array}$ & $2.9 \pm 0.2$ & $2.7 \pm 0.1$ & $2.9 \pm 0.3$ & $2.8 \pm 0.2$ & $3.0 \pm 0.3$ & $2.9 \pm 0.1$ & $2.9 \pm 0.2$ & $3.4 \pm 0.3$ \\
\hline $\begin{array}{c}\text { ChainA } \\
\text { H198 to } \\
\text { S306 }\end{array}$ & $4.1 \pm 0.2$ & $5.5 \pm 0.2$ & $4.2 \pm 0.2$ & $4.4 \pm 0.1$ & $4.2 \pm 0.2$ & $4.1 \pm 0.3$ & $4.1 \pm 0.2$ & $3.5 \pm 0.3$ \\
\hline
\end{tabular}




\begin{tabular}{|c|c|c|c|c|c|c|c|c|}
\hline $\begin{array}{c}\text { ChainB } \\
\text { H198 to } \\
\text { D228 }\end{array}$ & $2.9 \pm 0.2$ & $2.6 \pm 0.2$ & $2.9 \pm 0.3$ & $2.8 \pm 0.1$ & $3.0 \pm 0.3$ & $3.1 \pm 0.2$ & $2.9 \pm 0.2$ & $3.1 \pm 0.1$ \\
\hline $\begin{array}{c}\text { ChainB } \\
\text { H198 to } \\
\text { S306 }\end{array}$ & $4.1 \pm 0.2$ & $4.8 \pm 0.1$ & $4.2 \pm 0.2$ & $4.5 \pm 0.1$ & $4.2 \pm 0.2$ & $4.0 \pm 0.3$ & $4.1 \pm 0.2$ & $3.6 \pm 0.2$ \\
\hline $\begin{array}{c}\text { ChainC } \\
\text { H198 to } \\
\text { D228 }\end{array}$ & $2.9 \pm 0.2$ & $2.7 \pm 0.1$ & $2.9 \pm 0.3$ & $2.8 \pm 0.1$ & $3.0 \pm 0.3$ & $2.7 \pm 0.3$ & $2.9 \pm 0.2$ & $3.3 \pm 0.3$ \\
\hline $\begin{array}{c}\text { ChainC } \\
\text { H198 to } \\
\text { S306 }\end{array}$ & $4.1 \pm 0.2$ & $5.2 \pm 0.2$ & $4.2 \pm 0.2$ & $4.4 \pm 0.1$ & $4.2 \pm 0.2$ & $4.5 \pm 0.3$ & $4.1 \pm 0.2$ & $3.8 \pm 0.1$ \\
\hline
\end{tabular}

Table S3: Quantitative analyses of the active-site modification assay. The average fluorescence intensity values and the corresponding fold change for $\mathrm{W}$ and $\Delta$ subunit in the control and test samples for each HtrA2 variant.

(A) Substrate-induced activation

\begin{tabular}{|c|c|c|c|c|c|c|}
\hline \multirow{2}{*}{$\begin{array}{c}\text { HtrA2 } \\
\text { Variants }\end{array}$} & \multicolumn{3}{|c|}{ W Subunit } & \multicolumn{3}{|c|}{$\Delta$ Subunit } \\
\hline & Control* & Test* & $\begin{array}{c}\text { Fold } \\
\text { change }\end{array}$ & Control* & Test* & $\begin{array}{c}\text { Fold } \\
\text { change }\end{array}$ \\
\hline WWW & 4865 & 53518 & 11 & 0 & 0 & 0 \\
\hline WW & 11805 & 59415 & 5 & 7625.5 & 54542.5 & 7 \\
\hline $\mathrm{W} \Delta \Delta$ & 8629 & 35255 & 4 & 14469 & 46090 & 3 \\
\hline$\Delta \Delta \Delta$ & 0 & 0 & 0 & 2687 & 6648.5 & 2.5 \\
\hline
\end{tabular}

(B) Temperature-induced activation

\begin{tabular}{|c|c|c|c|c|c|c|}
\hline \multirow{2}{*}{$\begin{array}{c}\text { HtrA2 } \\
\text { Variants }\end{array}$} & \multicolumn{3}{|c|}{ W Subunit } & \multicolumn{3}{|c|}{$\Delta$ Subunit } \\
\hline & Control* & Test* & $\begin{array}{c}\text { Fold } \\
\text { change }\end{array}$ & Control* & Test* & $\begin{array}{c}\text { Fold } \\
\text { change }\end{array}$ \\
\hline WWW & 2682.5 & 6803.5 & 2.53 & 0 & 0 & 0 \\
\hline WW & 5954 & 14646 & 2.46 & 4436 & 10314 & 2.32 \\
\hline $\mathrm{W} \Delta \Delta$ & 5803.5 & 10582.5 & 1.82 & 10639.5 & 26644 & 2.5 \\
\hline$\Delta \Delta \Delta$ & 0 & 0 & 0 & 2456.5 & 7258 & 2.95 \\
\hline
\end{tabular}

* Arbitrary Unit 
Table S4: Crystallography data statistics for $\Delta \Delta \Delta$ (PDB ID: 7VGE)

\begin{tabular}{|c|c|}
\hline $\begin{array}{l}\text { Data collection and refinement } \\
\text { statistics }\end{array}$ & $\operatorname{HtrA2} \Delta \Delta \Delta$ \\
\hline \multicolumn{2}{|l|}{ Data collection statistics } \\
\hline Space group & $P 4_{3} 2_{1} 2$ \\
\hline $\begin{array}{l}\text { Unit cell parameters } \\
a, b, c(\AA) \\
\alpha, \beta, \gamma\left({ }^{\circ}\right)\end{array}$ & $\begin{array}{l}82.88,82.88,395.31 \\
90.00,90.00,90.00\end{array}$ \\
\hline Temperature (K) & 100 \\
\hline Wavelength $(\AA)$ & 1.5419 \\
\hline Resolution $(\AA)$ & $40.0-4.0(4.1-4.0)$ \\
\hline$R_{\text {merge }}(\%)$ & $39.8(144.8)$ \\
\hline Completeness (\%) & 90.7 (90.9) \\
\hline Mean $I / \sigma(I)$ & $4.36(1.27)$ \\
\hline Total reflections & 85456 (5452) \\
\hline Unique reflections & $20149(1441)$ \\
\hline Redundancy & $4.24(3.78)$ \\
\hline $\mathrm{CC}_{1 / 2}$ & $96.7(25.1)$ \\
\hline Wilson B factor $\left(\AA^{2}\right)$ & \begin{tabular}{|l|l|}
76.05 \\
\end{tabular} \\
\hline No. of molecules in ASU & 6 \\
\hline \multicolumn{2}{|l|}{ Refinement statistics } \\
\hline Resolution $(\AA)$ & $39.53-4.0$ \\
\hline Working set: no. of reflections & 11300 \\
\hline$R_{\text {factor }}(\%)$ & 26.51 \\
\hline Test set: no. of reflections & 595 \\
\hline$R_{\text {free }}(\%)$ & 33.64 \\
\hline Protein atoms & 8149 \\
\hline \multicolumn{2}{|l|}{ Isotropic average $B$-factor $(\AA)$} \\
\hline Protein & 116.3 \\
\hline \multicolumn{2}{|l|}{ Geometry statistics } \\
\hline R.m.s.d. bond length $(\AA)$ & 0.006 \\
\hline R.m.s.d. bond angle $\left(^{\circ}\right)$ & 1.5 \\
\hline \multicolumn{2}{|l|}{ Ramachandran plot (\%) } \\
\hline Most favoured region & 93.0 \\
\hline Allowed regions & 6.7 \\
\hline Outlier & 0.3 \\
\hline
\end{tabular}




\section{Figure S1}
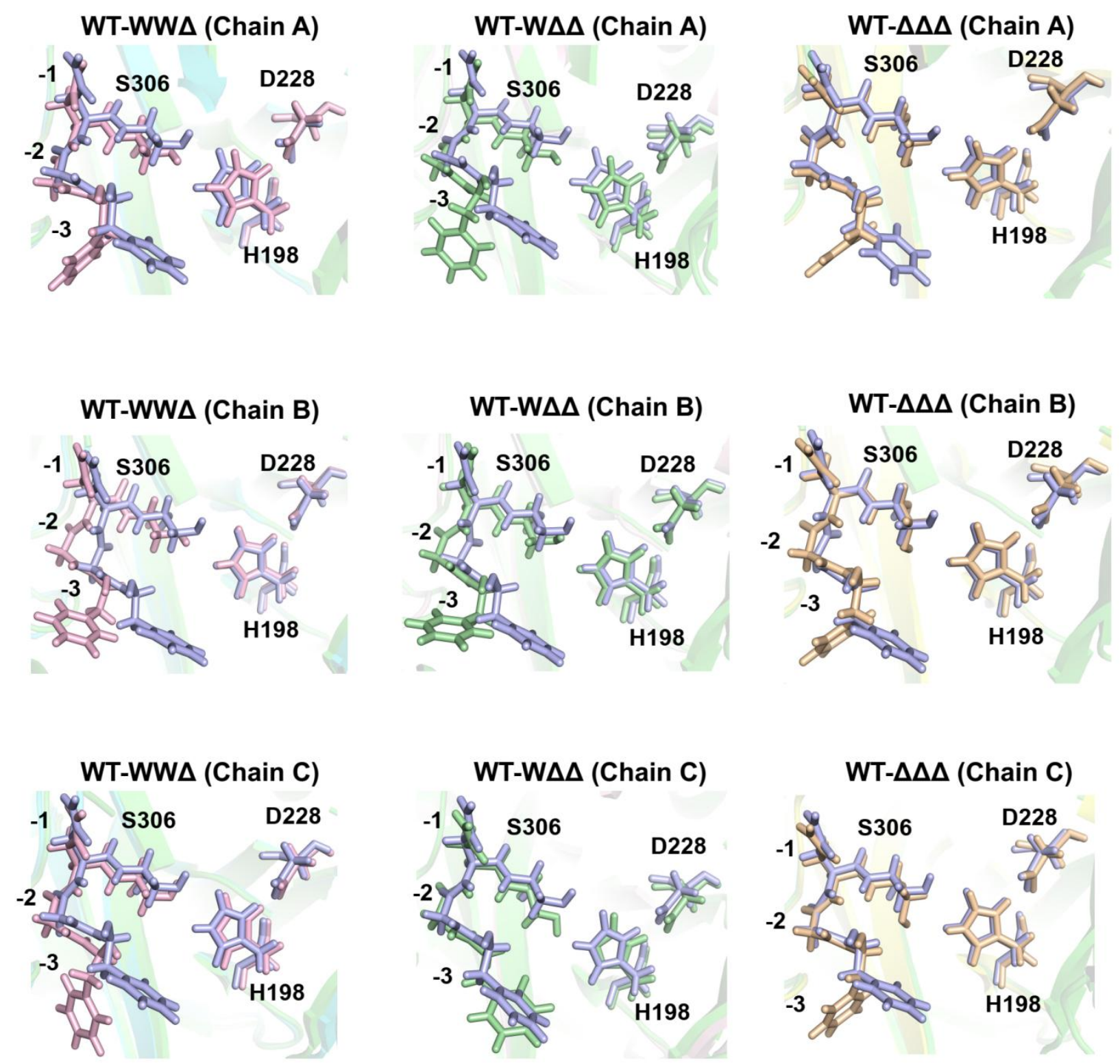

Figure S1: Orientation of the catalytic triad residues of HtrA2 variants. Stick diagram showing the alignment of the catalytic triad residues for heterotrimeric variants (WW $\Delta$ and $\mathrm{W} \Delta \Delta$ ) and $\Delta \Delta \Delta$ (N-SPD variant) with respect to the wild type (WWW). Catalytic residues are represented as H198, D228 and S306. Oxyanion hole residues are marked as $-1,-2$ and -3 
starting from the S306 residue which is considered as 0 . WWW, WW $\Delta, \mathrm{W} \Delta \Delta$ and $\Delta \Delta \Delta$ are denoted by light blue, pink, green and yellow sticks, respectively.

\section{Figure S2}

A

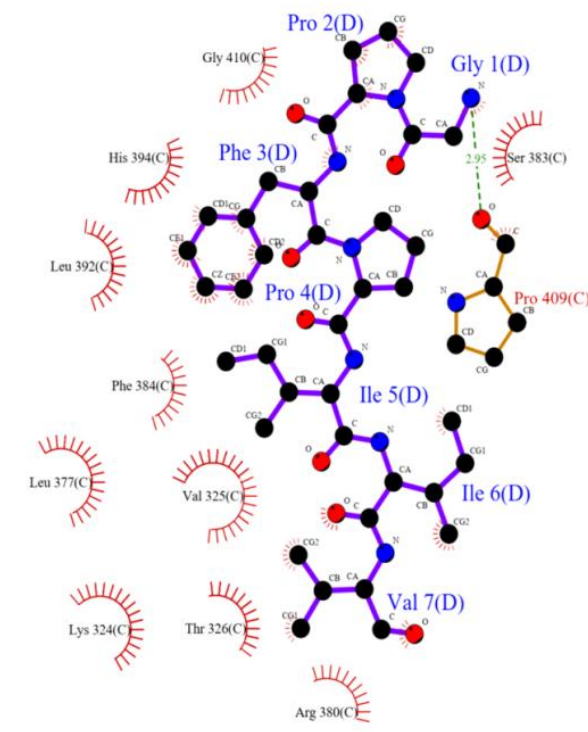

C

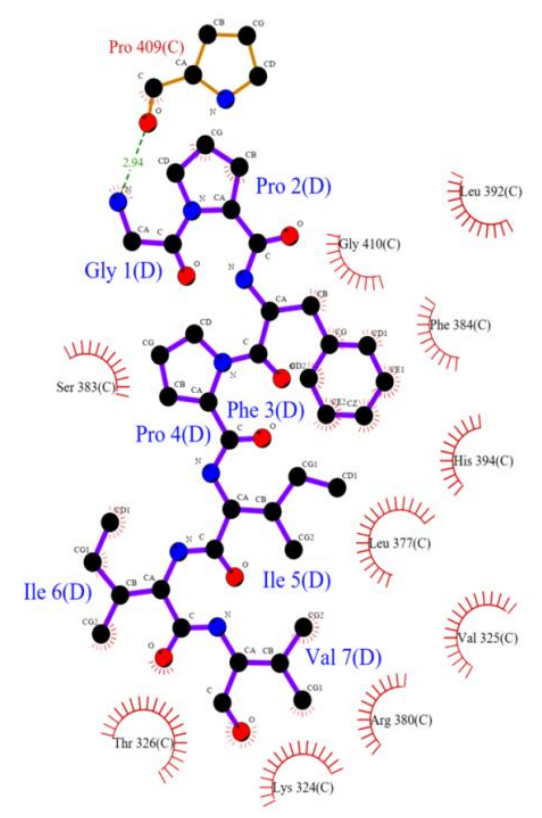

B

WWA-GPFPIIV

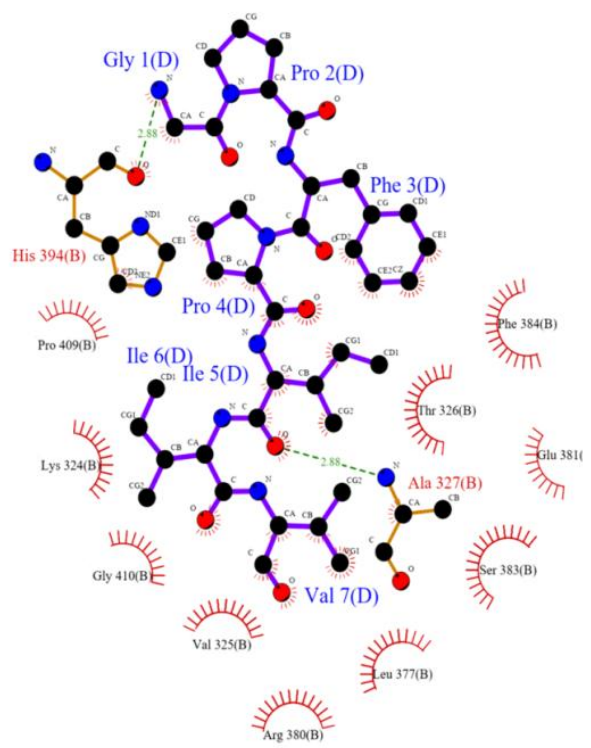

D $\Delta \Delta \Delta$-GPFPIIV

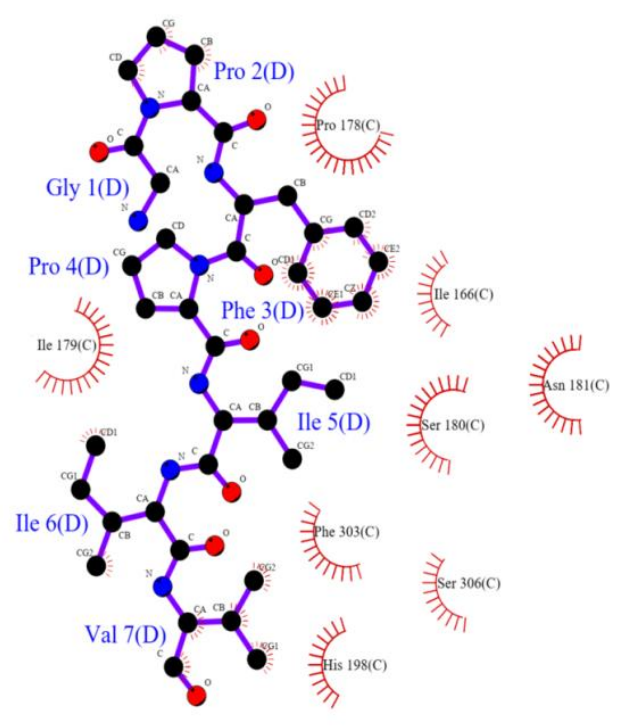


Figure S2: Interaction analyses of HtrA2 variants in presence of C-terminal ligand. Ligplot showing the interacting residues between the $\beta$-casein peptide (GPFPIIV) and A) WWW, B) WW $\Delta$, C) W $\Delta \Delta$ and D) $\Delta \Delta \Delta$ variants of HtrA2. Hydrogen bond interactions are denoted by green dotted lines.

\section{Figure S3}

A
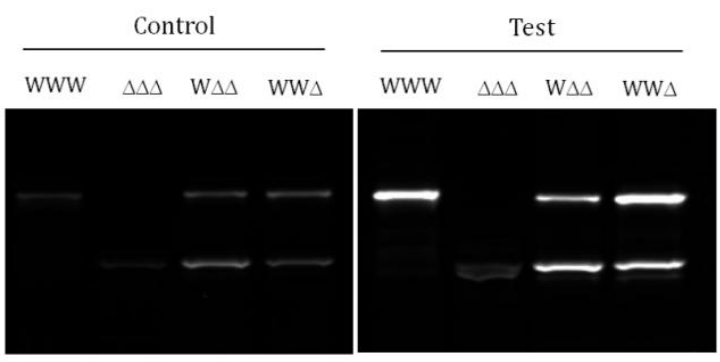

C

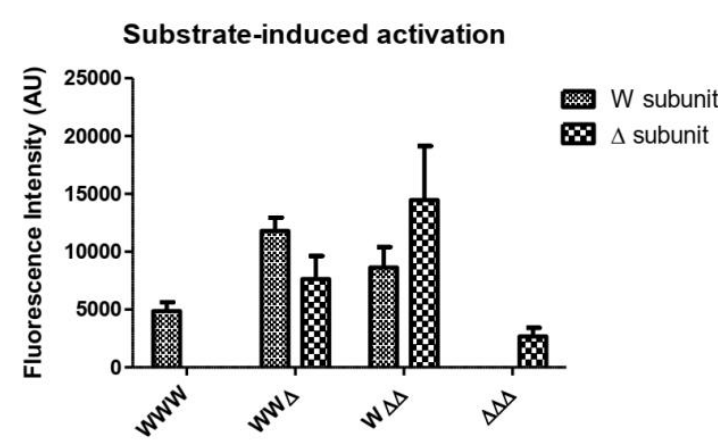

B

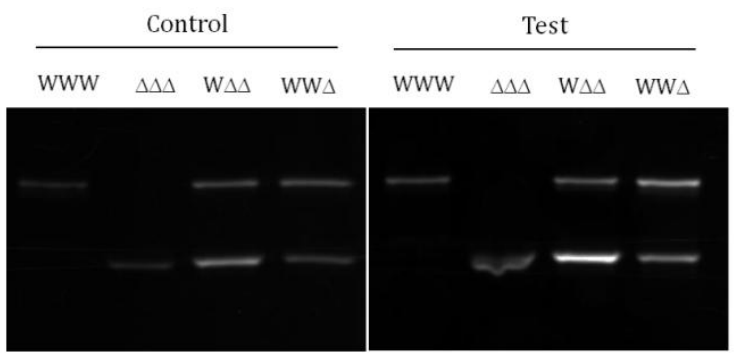

D

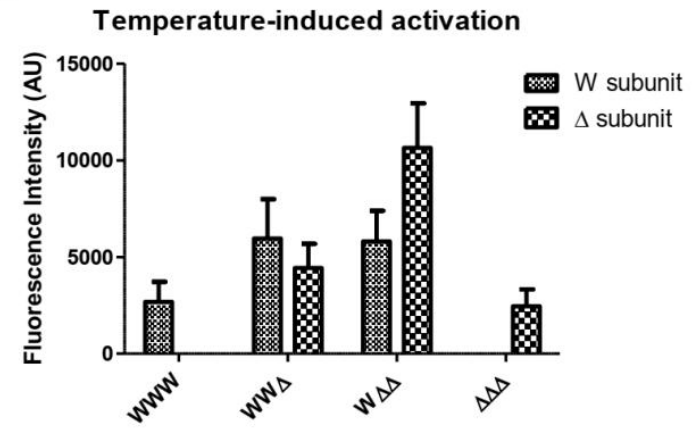

Figure S3: In-gel fluorescence imaging performed for active-site modification assay using

TAMRA-FP. Representative images from (A) Substrate-induced activation (B) Temperatureinduced activation. (C) The fluorescence intensities of the control samples in (A) were quantified using the Image Lab ${ }^{\mathrm{TM}}$ software (version 6.0.0 build 25). The values obtained for each subunit from multiple independent experiments were plotted using Graphpad Prism software with their 
respective SEM. (D) The fluorescence intensities of the control samples in (B) were quantified and represented in a similar way as in (C).

\section{Figure S4}
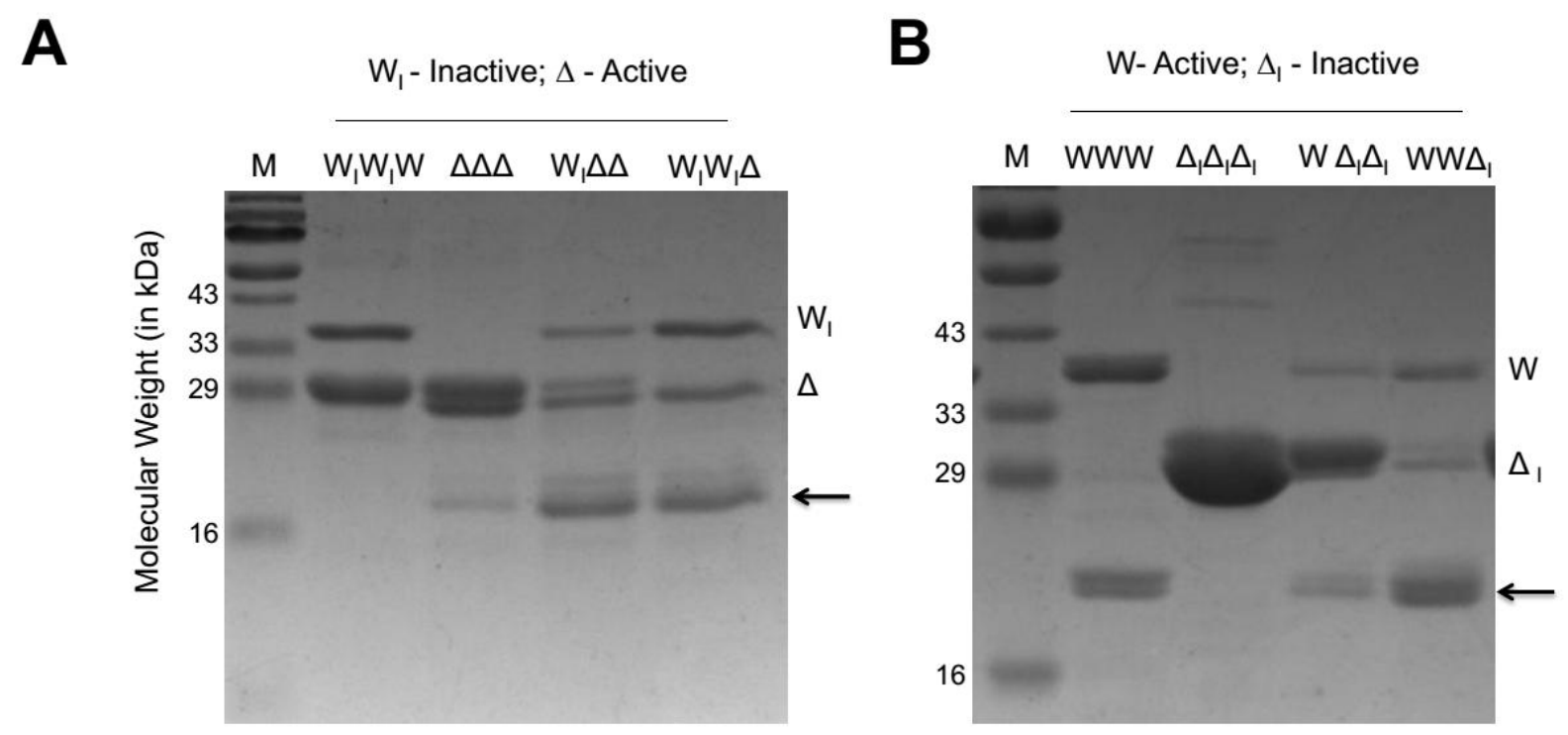

Figure S4: Proteolytic cleavage assays of HtrA2 variants with active-site mutation. (A) (B)

Qualitative gel-based proteolytic cleavage of $\beta$-casein by HtrA2 variants containing active-site mutation in different subunits. $2 \mu \mathrm{g}$ of each enzyme variant was incubated individually with $6 \mu \mathrm{g}$ of substrate $\beta$-casein and incubated at $37{ }^{\circ} \mathrm{C}$ for $30 \mathrm{~min}$. The reaction at each time point was stopped with Laemmli buffer at $100{ }^{\circ} \mathrm{C}$. Reaction samples were resolved by $12 \%$ SDS-PAGE and the cleavage pattern was visualized with Coomassie brilliant blue staining. Arrows indicate the cleaved products. M: Protein marker. 


\section{Figure S5}

\section{A}

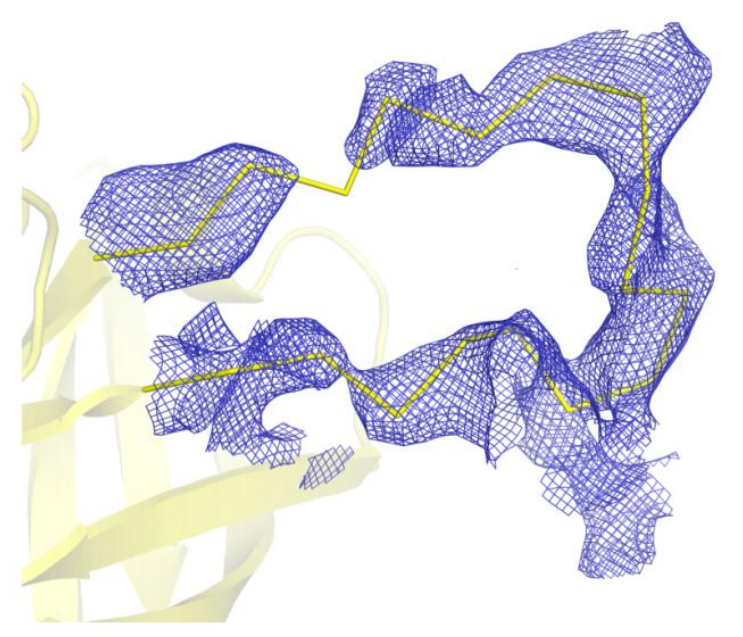

B

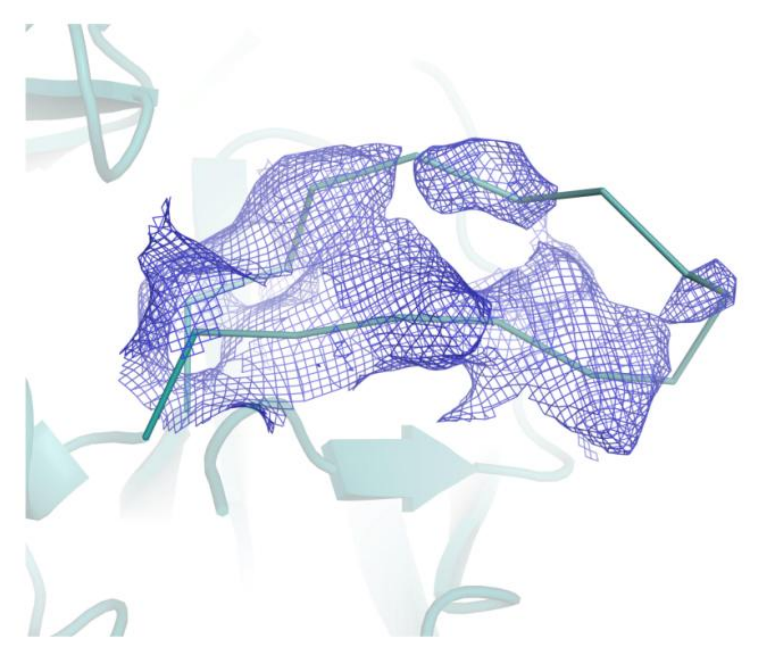

C

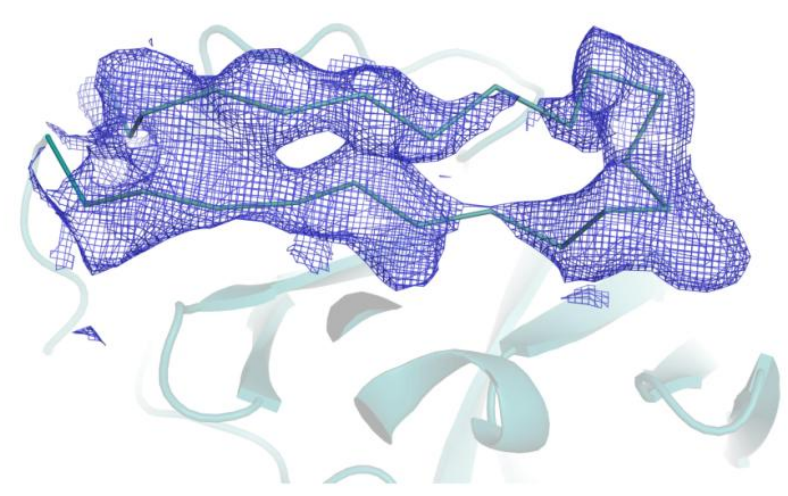

Figure S5: 2Fo-Fc electron density maps of regions comprising different loops in $\Delta \Delta \Delta$. The maps for (A) LA loop (165-181 residues, contoured at $0.8 \sigma$ ) from chain D, and the loops (B) L2 (320-333 residues, contoured at $0.8 \sigma$ ) and (C) LD (255-275 residues, contoured at $1.0 \sigma)$ from chain A are modeled. LA loop is represented as yellow ribbon, while L2 and LD loops are represented as deep olive ribbons. The map is displayed as blue mesh. 